\title{
The Algebraic Theory of Torsion. II: Products
}

\author{
ANDREW RANICKI \\ Department of Mathematics, University of Edinburgh, Edinburgh EH9 $3 J Z$, Scotland
}

(Received: 11 July 1986)

\begin{abstract}
The algebraic $K$-theory product $K_{0}(A) \otimes K_{1}(B) \rightarrow K_{1}(A \otimes B)$ for rings $A, B$ is given a chain complex interpretation, using the absolute torsion invariant introduced in Part $I$. Given a finitely dominated $A$-module chain complex $C$ and a round finite $B$-module chain complex $D$, it is shown that the $A \otimes B$-module chain complex $C \otimes D$ has a round finite chain homotopy structure. Thus, if $X$ is a finitely dominated $\mathrm{CW}$ complex and $Y$ is a round finite $\mathrm{CW}$ complex, the product $X \times Y$ is a CW complex with a round finite homotopy structure.
\end{abstract}

Key words. Finiteness obstruction, torsion, product, chain complex.

\section{Introduction}

The algebraic theory of absolute torsion developed in Part I ([16]) is here applied to products of chain complexes in algebra, and products of CW complexes in topology.

Given an additive category $\mathscr{A}$ and a chain equivalence $f: C \rightarrow D$ of finite chain complexes in $\mathscr{A}$ with $[C]=[D]=0 \in K_{0}(\mathscr{A})$ there was defined in Part I a torsion invariant $\tau(f) \in K_{1}^{\text {soo }}(\mathscr{A})$ in the isomorphism torsion group of $\mathscr{A}$. Here, we shall only be concerned with the case of the additive category $\mathscr{A}$ of based f.g. free $A$-modules, for some ring $A$ such that the rank of f.g. free $A$-modules is well-defined. Thus, the natural map $K_{0}(\mathbb{Z})=\mathbb{Z} \rightarrow K_{0}(A)$ is injective, and the Euler characteristic of a finite chain complex $C$ in $\mathscr{A}$

$$
\chi(C)=\sum_{r=0}^{\infty}(-)^{r} \operatorname{rank}\left(C_{r}\right) \in \mathbb{Z}
$$

is a chain homotopy invariant which can be identified with the class $[C] \in K_{0}(\mathscr{A})=\mathbb{Z}$, and also the projective class $[C] \in K_{0}(A)$. Isomorphic objects in $\mathscr{A}$ are related by a canonical isomorphism, so there is defined a natural split surjection $K_{1}^{\text {iso }}(\mathscr{A}) \rightarrow K_{1}^{\text {aut }}(\mathscr{A})=K_{1}(A)$. Given a chain equivalence $f: C \rightarrow D$ of finite chain complexes of based f.g. free $A$-modules such that $\chi(C)=\chi(D)=0 \in \mathbb{Z}$ we thus have an invariant $\tau(f) \in K_{1}(A)$, the torsion of $f$. The definition of $\tau(f)$ is recalled in Section 1 below.

The absolute projective class of a finitely dominated $\mathrm{CW}$ complex $X$ is defined to be the projective class of the finitely dominated cellular $\mathbb{Z}\left[\pi_{1}(X)\right]$-module chain complex $C(\tilde{X})$ of the universal cover $\tilde{X}$

$$
[X]=[C(\tilde{X})] \in K_{0}\left(\mathbb{Z}\left[\pi_{1}(X)\right]\right),
$$

and consists of the Euler characteristic $\chi(X)=\chi(C(\tilde{X})) \in K_{0}(\mathbb{Z})=\mathbb{Z}$ and the finite- 
ness obstruction $[\tilde{X}] \in \tilde{K}_{0}\left(\mathbb{Z}\left[\pi_{1}(X)\right]\right)$ of Wall $[21]$

$$
[X]=(\chi(X),[\tilde{X}]) \in K_{0}\left(\mathbb{Z}\left[\pi_{1}(X)\right]\right)=K_{0}(\mathbb{Z}) \oplus \tilde{K}_{0}\left(\mathbb{Z}\left[\pi_{1}(X)\right]\right) .
$$

The product of finitely dominated $\mathrm{CW}$ complexes $X, Y$ is a finitely dominated $\mathrm{CW}$ complex $X \times Y$ with universal cover $\widetilde{X \times Y}=\tilde{X} \times \tilde{Y}$, such that

$$
\mathbb{Z}\left[\pi_{1}(X \times Y)\right]=\mathbb{Z}\left[\pi_{1}(X) \times \pi_{1}(Y)\right]=\mathbb{Z}\left[\pi_{1}(X)\right] \otimes \mathbb{Z}\left[\pi_{1}(Y)\right],
$$

with a natural identification

$$
C(\overparen{X \times Y})=C(\tilde{X}) \otimes C(\tilde{Y}) .
$$

The projective class product formula of Gersten [7] and Siebenmann [18]

$$
[X \times Y]=[X] \otimes[Y] \in K_{0}\left(\mathbb{Z}\left[\pi_{1}(X \times Y)\right]\right)
$$

showed that for a finite CW complex $Y$ with $\chi(Y)=0 \in \mathbb{Z}$ the product $X \times Y$ has Wall finiteness obstruction $[\widetilde{X \times Y}]=0 \in \tilde{K}_{0}\left(\mathbb{Z}\left[\pi_{1}(X \times Y)\right]\right)$, and so $X \times Y$ has the homotopy type of a finite $\mathrm{CW}$ complex. This was first proved geometrically by Mather [12], in the important special case $Y=S^{1}$.

For any rings $A, B$ there is defined a product in the absolute algebraic $K$-groups

$$
\begin{aligned}
& \otimes: K_{0}(A) \otimes K_{1}(B) \rightarrow K_{1}(A \otimes B), \\
& {[P] \otimes \tau(f: Q \rightarrow Q) \mapsto \tau(1 \otimes f: P \otimes Q \rightarrow P \otimes Q),}
\end{aligned}
$$

in particular for group rings $A=\mathbb{Z}[\pi], B=\mathbb{Z}[\rho]$, with $A \otimes B=\mathbb{Z}[\pi \times \rho]$. In general, there is no such product in the reduced $K$-groups, although if $W h(\rho)=0$ there is a product $\widetilde{K}_{0}(\mathbb{Z}[\pi]) \otimes K_{1}(\mathbb{Z}[\rho]) \rightarrow W h(\pi \times \rho)$. It is therefore quite reasonable that the absolute torsion should enter into the consideration of finite $\mathrm{CW}$ complexes in the homotopy type of CW complex products $X \times Y$.

Define a finite structure on a CW complex $X$ to be an equivalence class of pairs

(finite CW complex $F$, homotopy equivalence $\phi: F \rightarrow X$ )

under the equivalence relation

$$
\left(F_{1}, \phi_{1}\right) \sim\left(F_{2}, \phi_{2}\right) \quad \text { if } \quad \tau\left(\phi_{2}^{-1} \phi_{1}: F_{1} \rightarrow F_{2}\right)=0 \in \mathrm{Wh}\left(\pi_{1}(X)\right) .
$$

The Whitehead torsion $\tau(f) \in \mathrm{Wh}\left(\pi_{1}(X)\right)$ of a homotopy equivalence $f: X \rightarrow X^{\prime}$ of $\mathrm{CW}$ complexes with given finite structures $(F, \phi),\left(F^{\prime}, \phi^{\prime}\right)$ is defined by

$$
\tau(f)=\tau\left(\phi^{\prime-1} f \phi: F \rightarrow X \rightarrow X^{\prime} \rightarrow F^{\prime}\right) \in \mathrm{Wh}\left(\pi_{1}(X)\right) .
$$

A finite CW complex $F$ has the canonical finite structure $(F, 1)$.

Ferry [6] proved geometrically that the mapping torus construction of Mather [12] defines a canonical finite structure on $X \times S^{1}$ for any finitely dominated CW complex $X$, which is independent of the finite domination used in the construction, and that the geometrically defined Abelian group morphism

$$
\bar{B}^{\prime}: \tilde{K}_{0}(\mathbb{Z}[\pi]) \rightarrow \mathrm{Wh}(\pi \times \mathbb{Z}) ;[X] \mapsto \tau\left(1 \times-1: X \times S^{1} \rightarrow X \times S^{1}\right)\left(\pi=\pi_{1}(X)\right)
$$


is an injection. Now $-1: S^{1} \rightarrow S^{1}$ is a simple homotopy equivalence (i.e., $\left.\tau(-1)=0 \in \mathrm{Wh}\left(\pi_{1}\left(S^{1}\right)\right)=\mathrm{Wh}(\mathbb{Z})=0\right)$, so that the canonical finite structure on $X \times S^{1}$ depends on more than just the canonical finite structure on $S^{1}$. We shall show that it depends on the canonical 'round finite structure' on $S^{1}$.

A finite chain complex $C$ of based f.g. free $A$-modules is round if $\chi(C)=0 \in \mathbb{Z}$, or equivalently if $[C]=0 \in K_{0}(A)$. The torsion $\tau(f) \in K_{1}(A)$ defined in Part I for a chain equivalence $f: C \rightarrow D$ of round finite chain complexes has the logarithmic property

$$
\tau(g f: C \rightarrow D \rightarrow E)=\tau(f: C \rightarrow D)+\tau(g: D \rightarrow E) \in K_{1}(A) .
$$

In general, absolute torsion is nonadditive

$$
\tau\left(f \oplus f^{\prime}: C \oplus C^{\prime} \rightarrow D \oplus D^{\prime}\right) \neq \tau(f: C \rightarrow D)+\tau\left(f^{\prime}: C^{\prime} \rightarrow D^{\prime}\right) \in K_{1}(A) .
$$

A round finite structure on an $A$-module chain complex $C$ is an equivalence class of pairs

(round finite chain complex $F$ of based f.g. free $A$-modules, chain equivalence $\phi: F \rightarrow C$ )

under the equivalence relation

$$
\left(F_{1}, \phi_{1}\right) \sim\left(F_{2}, \phi_{2}\right) \text { if } \tau\left(\phi_{2}^{-1} \phi_{1}: F_{1} \rightarrow F_{2}\right)=0 \in K_{1}(A) .
$$

The torsion of a chain equivalence $f: C \rightarrow C^{\prime}$ of $A$-module chain complexes $C, C^{\prime}$ with prescribed round finite structures $(F, \phi),\left(F^{\prime}, \phi^{\prime}\right)$ is defined by

$$
\tau(f)=\tau\left(\phi^{\prime-1} f \phi: F \rightarrow C \rightarrow C^{\prime} \rightarrow F^{\prime}\right) \in K_{1}(A) .
$$

The main result of the paper is the following chain complex interpretation of the product $K_{0}(A) \otimes K_{1}(B) \rightarrow K_{1}(A \otimes B)$.

ALGEBRAIC PRODUCT STRUCTURE THEOREM. The product of a finitely dominated $A$-module chain complex $C$ and a $B$-module chain complex $D$ with a round finite structure $(F, \phi)$ is an $A \otimes B$-module chain complex $C \otimes D$ with a round finite structure $C \otimes(F, \phi)$.

If $f: C \rightarrow C^{\prime}$ is a chain equivalence of finitely dominated A-module chain complexes and $g: D \rightarrow D^{\prime}$ is a chain equivalence of $B$-module chain complexes $D, D^{\prime}$ with round finite structures

$$
\tau\left(f \otimes g: C \otimes D \rightarrow C^{\prime} \otimes D^{\prime}\right)=[C] \otimes \tau(g) \in K_{1}(A \otimes B)
$$

with $[C]=\left[C^{\prime}\right] \in K_{0}(A)$ the projective class and $\tau(g) \in K_{1}(B)$ the torsion.

This will be proved in Section 3, and translated into topology in Section 4.

A finite CW complex $X$ with universal cover $\widetilde{X}$ and fundamental group $\pi_{1}(X)=\pi$ determines a class of bases for the cellular f.g. free $\mathbb{Z}[\pi]$-module chain complex $C(\tilde{X})$, the elements of which are determined up to multiplication by $\pm g \in \mathbb{Z}[\pi]$ $(g \in \pi)$. Define a round finite $C W$ complex to be a finite CW complex $X$ such that the Euler characteristic $\chi(X)=\chi(C(\bar{X})) \in \mathbb{Z}$ vanishes, $\chi(X)=0 \in \mathbb{Z}$, together with a choice 
of base for $C(\tilde{X})$ in the canonical class. The torsion of a homotopy equivalence $f: X \rightarrow Y$ of round finite $\mathrm{CW}$ complexes is defined by

$$
\tau(f)=\tau(\tilde{f}: C(\tilde{X}) \rightarrow C(\tilde{Y})) \in K_{1}(\mathbb{Z}[\pi]) \quad\left(\pi=\pi_{1}(X)\right),
$$

with the image $\tau(f) \in \mathrm{Wh}(\pi)$ the usual Whitehead torsion of $f$.

A round finite structure on a CW complex $X$ is a round finite structure on $C(\tilde{X})$, or equivalently an equivalence class of pairs

(round finite $\mathrm{CW}$ complex $F$, homotopy equivalence $\phi: F \rightarrow X$ )

under the equivalence relation

$$
\left(F_{1}, \phi_{1}\right) \sim\left(F_{2}, \phi_{2}\right) \quad \text { if } \tau\left(\phi_{2}^{-1} \phi_{1}: F_{1} \rightarrow F_{2}\right)=0 \in K_{1}\left(\mathbb{Z}\left[\pi_{1}(X)\right]\right) .
$$

The torsion $\tau(f) \in K_{1}\left(\mathbb{Z}\left[\pi_{1}(X)\right]\right)$ of a homotopy equivalence $f: X \rightarrow Y$ of $\mathrm{CW}$ complexes with prescribed round finite structures is defined in the obvious manner.

The main topological result of this paper is the following CW complex interpretation of the product $K_{0}(A) \otimes K_{1}(B) \rightarrow K_{1}(A \otimes B)$.

GEOMETRIC PRODUCT STRUCTURE THEOREM. The product of a finitely dominated $C W$ complex $X$ and a $C W$ complex $Y$ with round finite structure $(F, \phi)$ is a $C W$ complex $X \times Y$ with a round finite structure $X \times(F, \phi)$. If $f: X \rightarrow X^{\prime}$ is a homotopy equivalence of finitely dominated $C W$ complexes and $g: Y \rightarrow Y^{\prime}$ is a homotopy equivalence of $C W$ complexes with round finite structures then

$$
\tau\left(f \times g: X \times Y \rightarrow X^{\prime} \times Y^{\prime}\right)=[X] \otimes \tau(g) \in K_{1}\left(\mathbb{Z}\left[\pi_{1}(X \times Y)\right]\right),
$$

with $[X]=\left[X^{\prime}\right] \in K_{0}\left(\mathbb{Z}\left[\pi_{1}(X)\right]\right)$ the projective class and $\tau(g) \in K_{1}\left(\mathbb{Z}\left[\pi_{1}(Y)\right]\right)$ the torsion.

The torsion product formulae of Kwun and Szczarba [10] and Gersten [8] are special cases of the geometric product structure theorem, with $X$ finite in $[10]$ and $Y^{\prime}=Y$ in [8].

As already noted in the introduction to Part I ([16]), the algebraic description due to Lück [11] of the transfer maps induced in the algebraic $K$-groups

$$
p_{i}^{\prime}: K_{i}\left(\mathbb{Z}\left[\pi_{1}(B)\right]\right) \rightarrow K_{i}\left(\mathbb{Z}\left[\pi_{1}(E)\right]\right) \quad(i=0,1)
$$

by a Hurewicz fibration

$$
F \rightarrow E \stackrel{p}{\longrightarrow} B
$$

with finitely dominated fibre $F$ allows the extension of the geometric product structure theorem to the twisted case: if the base $B$ is also finitely dominated then so is the total space $E$, with projective class

$$
[E]=p_{0}^{\prime}([B]) \in K_{0}\left(\mathbb{Z}\left[\pi_{1}(E)\right]\right),
$$

and a round finite structure on $B$ determines a round finite structure on $E$, a variation by $\tau \in K_{1}\left(\mathbb{Z}\left[\pi_{1}(B)\right]\right)$ in the base leading to a variation of 
$p_{1}^{\prime}(\tau) \in K_{1}\left(\mathbb{Z}\left[\pi_{1}(E)\right]\right)$ in the total space. In the case of a trivial fibration $E=B \times F$ the transfer maps are given by product with the projective class $[F] \in K_{0}\left(\mathbb{Z}\left[\pi_{1}(F)\right]\right)$

$$
p_{i}^{\prime}=-\otimes[F]: K_{i}\left(\mathbb{Z}\left[\pi_{1}(B)\right]\right) \rightarrow K_{i}\left(\mathbb{Z}\left[\pi_{1}(B \times F)\right]\right) \quad(i=0,1) .
$$

In Section 5 we shall compare the absolute torsion invariant $\tau(f) \in K_{1}\left(\mathbb{Z}\left[\pi_{1}(X)\right]\right)$ defined by Gersten [8] for a self homotopy equivalence $f: X \rightarrow X$ of a finitely dominated CW complex $X$ with $f_{*}=1: \pi_{1}(X) \rightarrow \pi_{1}(X)$ with our notion of absolute torsion, showing that they coincide when both are defined (i.e., when $\left.[X]=0 \in K_{0}\left(\mathbb{Z}\left[\pi_{1}(X)\right]\right)\right)$.

Finally, in Section 6 we shall show that for a particular choice of round finite structure $\Sigma^{1}$ on $S^{1}$ the product round finite structure $X \times \Sigma^{1}$ on $X \times S^{1}$ for a finitely dominated CW complex $X$ reduces to the canonical finite structure obtained geometrically by Mather [12] and Ferry [6]. With respect to this choice

$$
\begin{aligned}
\tau( & \left.-1: S^{1} \rightarrow S^{1}\right) \\
& =\tau\left(-z: \mathbb{Z}\left[z, z^{-1}\right] \rightarrow \mathbb{Z}\left[z, z^{-1}\right]\right) \in K_{1}\left(\mathbb{Z}\left[\pi_{1}\left(S^{1}\right)\right]\right) \\
& =K_{1}\left(\mathbb{Z}\left[z, z^{-1}\right]\right),
\end{aligned}
$$

so that the geometric injection of [6]

$$
\bar{B}^{\prime}: \tilde{K}_{0}(\mathbb{Z}[\pi]) \rightarrow \mathrm{Wh}(\pi \times \mathbb{Z}) ; \quad[X] \mapsto \tau\left(1 \times-1: X \times S^{1} \rightarrow X \times S^{1}\right)
$$

may be identified with the algebraic injection of Ranicki [22]

$$
\begin{array}{r}
\bar{B}^{\prime}=-\otimes \tau(-z): \tilde{K}_{0}(\mathbb{Z}[\pi]) \rightarrow \mathrm{Wh}(\pi \times \mathbb{Z}) ; \\
{[P] \mapsto \tau\left(-z: P\left[z, z^{-1}\right] \rightarrow P\left[z, z^{-1}\right]\right) .}
\end{array}
$$

Thus, $\bar{B}^{\prime}$ is a variant of the algebraic injection defined by Bass et al. [2]

$$
\begin{array}{r}
\bar{B}=-\otimes \tau(z): \tilde{K}_{0}(\mathbb{Z}[\pi]) \rightarrow \mathrm{Wh}(\pi \times \mathbb{Z}) ; \\
{[P] \mapsto \tau\left(z: P\left[z, z^{-1}\right] \rightarrow P\left[z, z^{-1}\right]\right) .}
\end{array}
$$

Part III of the paper [17] deals with lower $K$-theory, including some further discussion of $\bar{B}$ and $\bar{B}^{\prime}$.

See [9] for an application of the algebraic theory of torsion to $L$-theory.

\section{Finite and Round Finite Structures}

We shall now apply the general theory of torsion developed in Part I for any additive category to the most important special case $\mathscr{A}=\{$ based f.g. free $A$ modules $\}$, for any ring $A$ such that the rank of f.g. free $A$-modules is well-defined. In the first instance we recall from [15] the abstract chain complex version of the finiteness obstruction theory of Wall [21], and extend it to round finiteness.

A chain complex over $A$ is a positive chain complex of (left) $A$-modules and $A$ module morphisms

$$
C: \cdots \rightarrow C_{r+1} \stackrel{d}{\longrightarrow} C_{r} \stackrel{d}{\rightarrow} C_{r-1} \rightarrow \cdots \rightarrow C_{1} \stackrel{d}{\longrightarrow} C_{0}
$$


The chain complex $C$ is $n$-dimensional if $C_{r}=0$ for $r>n$. The chain complex $C$ is finite if it is a finite-dimensional complex of based f.g. free $A$-modules, that is if it is finite chain complex in the category $\mathscr{A}=$ \{based f.g. free $A$-modules $\}$. The Euler characteristic of a finite chain complex $C$ is defined by

$$
\chi(C)=\sum_{r=0}^{\infty}(-)^{r} \operatorname{rank}_{A}\left(C_{r}\right) \in \mathbb{Z},
$$

and $C$ is round if $\chi(C)=0 \in \mathbb{Z}$.

A finite domination $(D, f, g, h)$ of a chain complex $C$ over $A$ consists of a finite chain complex $D$ over $A$, chain maps

$$
f: C \rightarrow D, \quad g: D \rightarrow C
$$

and a chain homotopy

$$
h: g f \simeq 1: C \rightarrow C \text {. }
$$

A chain complex is finitely dominated if it admits a finite domination. It was shown in [15] that a chain complex $C$ is finitely dominated if and only if it is chain equivalent to a finite dimensional f.g. projective chain complex

$$
P: \cdots \rightarrow 0 \rightarrow P_{n} \rightarrow P_{n-1} \rightarrow \cdots \rightarrow P_{1} \rightarrow P_{0} .
$$

The projective class of a finitely dominated chain complex $C$ is defined using any such $P$ to be

$$
[C]=[P]=\sum_{r=0}^{\infty}(-)^{r}\left[P_{r}\right] \in K_{0}(A) .
$$

The projective class is a chain homotopy invariant such that for finite $C$

$$
[C]=\chi(C) \in \operatorname{im}\left(K_{0}(\mathbb{Z}) \rightarrow K_{0}(A)\right)=\mathbb{Z} \subseteq K_{0}(A) .
$$

Thus the reduced projective class

$$
[C] \in \widehat{K}_{0}(A)=\operatorname{coker}\left(K_{0}(\mathbb{Z}) \rightarrow K_{0}(A)\right)
$$

vanishes for finite $C$.

PROPOSITION 1.1. (i) A finitely dominated chain complex $C$ over $A$ is chain equivalent to a finite chain complex if and only if $[C]=0 \in \tilde{K}_{0}(A)$. Thus $[C] \in \tilde{K}_{0}(A)$ is the finiteness obstruction of $C$.

(ii) A finitely dominated chain complex $C$ over $A$ is chain equivalent to a round finite chain complex if and only if $[C]=0 \in K_{0}(A)$. Thus $[C] \in K_{0}(A)$ is the round finiteness obstruction of $C$.

Proof. (i) See [1.5]. (ii) Immediate from (i).

The torsion of a contractible finite chain complex $C$ over $A$ is defined by

$$
\tau(C)=\tau\left(d+\Gamma=\left(\begin{array}{cccc}
d & 0 & 0 & \cdots \\
\Gamma & d & 0 & \cdots \\
0 & \Gamma & d & \cdots \\
\vdots & \vdots & \vdots &
\end{array}\right)\right.
$$


THE ALGEBRAIC THEORY OF TORSION. II: PRODUCTS

$$
\left.C_{\text {odd }}=C_{1} \oplus C_{3} \oplus C_{5} \oplus \cdots \rightarrow C_{\text {even }}=C_{0} \oplus C_{2} \oplus C_{4} \oplus \cdots\right) \in K_{1}(A)
$$

as usual, with $\Gamma: 0 \simeq 1: C \rightarrow C$ any chain contraction of $C$.

The algebraic mapping cone of a chain map $f: C \rightarrow D$ of finite chain complexes over $A$ is the finite chain complex $C(f)$ defined as usual (up to sign conventions) by

$$
d_{C(f)}=\left(\begin{array}{ll}
d_{D} & (-)^{r-1} f \\
0 & d_{C}
\end{array}\right): C(f)_{r}=D_{r} \oplus C_{r-1} \rightarrow C(f)_{r-1}=D_{r-1} \oplus C_{r-2}
$$

The following signs occur in the composition and sum formulae obtained in Part I [16], as recalled in Proposition 1.2 below.

Given based f.g. free $A$-modules $M, N$ let

$$
\varepsilon(M, N)=\operatorname{rank}_{A}(M) \operatorname{rank}_{A}(N) \in \mathbb{Z}_{2},
$$

so that

$$
\tau\left(\begin{array}{ll}
0 & 1 \\
1 & 0
\end{array}\right): M \oplus N \rightarrow N \oplus M=\varepsilon(M, N) \tau(-1: A \rightarrow A) \in K_{1}(A) .
$$

Given finite chain complexes $C, D$ over $A$ let

$$
\beta(C, D)=\Sigma_{i>j}\left(\varepsilon\left(C_{2 i}, D_{2 j}\right)+\varepsilon\left(C_{2 i+1}, D_{2 j+1}\right)\right) \in \mathbb{Z}_{2} .
$$

For any $A$-module chain complex $C$ let $S C$ denote the $A$-module chain complex with

$$
d_{S C}=d_{C}: S C_{r}=C_{r-1} \rightarrow S C_{r-1}=C_{r-2}
$$

Given finite chain complexes $C, D, E$ over $A$ let

$$
\begin{aligned}
& \gamma(C, D, E) \\
&= \beta(E, S C)-\beta(D, S C)-\beta(E, S D)+ \\
&+\left(\varepsilon\left(D_{\text {even }}, C_{\text {odd }}\right)-\varepsilon\left(D_{\text {odd }}, C_{\text {even }}\right)\right)+\left(\varepsilon\left(D_{\text {even }}, E_{\text {even }}\right)-\varepsilon\left(D_{\text {odd }}, E_{\text {odd }}\right)\right)+ \\
&+\left(\varepsilon\left(C_{\text {odd }}, E_{\text {even }}\right)-\varepsilon\left(C_{\text {even }}, E_{\text {odd }}\right)\right)+\left(\varepsilon\left(D_{\text {even }}, D_{\text {odd }}\right)-\varepsilon\left(D_{\text {even }}, D_{\text {even }}\right)\right) \in \mathbb{Z}_{2} .
\end{aligned}
$$

PROPOSITION 1.2. (i) The torsion of the algebraic mapping cone $C(g f)$ of the composite of $: C \rightarrow E$ of chain equivalences $f: C \rightarrow D, g: D \rightarrow E$ of finite chain complexes over $A$ is given by

$$
\tau(C(g f))=\tau(C(f))+\tau(C(g))+\gamma(C, D, E) \tau(-1: A \rightarrow A) \in K_{1}(A) .
$$

(ii) The torsion of the algebraic mapping cone $C\left(f \oplus f^{\prime}\right)$ of the sum $f \oplus f^{\prime}: C \oplus C^{\prime} \rightarrow D \oplus D^{\prime}$ of chain equivalences $f: C \rightarrow D, f^{\prime}: C^{\prime} \rightarrow D^{\prime}$ of finite chain complexes over $A$ is given by

$$
\begin{aligned}
\tau\left(C\left(f \oplus f^{\prime}\right)\right)= & \tau(C(f))+\tau\left(C\left(f^{\prime}\right)\right)+\beta\left(D \oplus S C, D^{\prime} \oplus S C^{\prime}\right) \tau(-1: A \rightarrow A)+ \\
& +\left(\Sigma_{r}(-)^{r} \varepsilon\left(C_{r-1}, D_{r}^{\prime}\right)\right) \tau(-1: A \rightarrow A) \in K_{1}(A) .
\end{aligned}
$$

Proof. See Proposition 2.5 of Part I. 
The reduced torsion of a chain equivalence $f: C \rightarrow D$ of finite chain complexes over $A$ is defined by

$$
\tau(f)=\tau(C(f)) \in \widehat{K}_{1}(A),
$$

the reduction of $\tau(C(f)) \in K_{1}(A)$ in $\tilde{K}_{1}(A)=\operatorname{coker}\left(K_{1}(\mathbb{Z}) \rightarrow K_{1}(A)\right)$.

PROPOSITION 1.3. The reduced torsion is such that

(i) $\tau(g f: C \rightarrow D \rightarrow E)=\tau(f)+\tau(g) \in \tilde{K}_{1}(A)$

(ii) $\tau\left(f \oplus f^{\prime}: C \oplus C^{\prime} \rightarrow D \oplus D^{\prime}\right)=\tau(f)+\tau\left(f^{\prime}\right) \in \tilde{K}_{1}(A)$

(iii) $\tau(f: C \rightarrow D)=\tau(D)-\tau(C) \in \widetilde{K}_{1}(A)$ if $C$ and $D$ are chain contractible.

Proof. See Proposition 2.6 of Part I.

The torsion of a chain equivalence $f: C \rightarrow D$ of round finite chain complexes over $A$ is defined by

$$
\tau(f)=\tau(C(f))-\beta(D, S C) \tau(-1: A \rightarrow A) \in K_{1}(A) .
$$

PROPOSITION 1.4. The torsion is such that

(i) $\tau(g f: C \rightarrow D \rightarrow E)=\tau(f)+\tau(g) \in K_{1}(A)$,

(ii) $\tau\left(f \oplus f^{\prime}: C \oplus C^{\prime} \rightarrow D \oplus D^{\prime}\right)$

$$
\begin{aligned}
= & \tau(f)+\tau\left(f^{\prime}\right)+ \\
& +\left(\beta\left(D, D^{\prime}\right)-\beta\left(C, C^{\prime}\right)\right) \tau(-1: A \rightarrow A) \in K_{1}(A),
\end{aligned}
$$

(iii) $\tau(f: C \rightarrow D)=\tau(D)-\tau(C) \in K_{1}(A)$ if $C$ and $D$ are chain contractible.

Proof. See Proposition 2.7 of Part I.

The reduction of the torsion $\tau(f) \in K_{1}(A)$ is, of course, the reduced torsion $\tau(f) \in \widetilde{K}_{1}(A)$.

A finite structure on a chain complex $C$ over $A$ is an equivalence class of pairs

(finite chain complex $F$ over $A$, chain equivalence $\phi: F \rightarrow C$ )

under the equivalence relation

$$
(F, \phi) \sim\left(F^{\prime}, \phi^{\prime}\right) \quad \text { if } \tau\left(\phi^{\prime-1} \phi: F \rightarrow F^{\prime}\right)=0 \subseteq \tilde{K}_{1}(A) .
$$

The finite structure set $\mathscr{F}(C)$ of a chain complex $C$ over $A$ is the set (possibly empty) of finite structures on $C$.

PROPOSITION 1.5. (i) The finite structure set $\mathscr{F}(C)$ is nonempty if and only if $C$ is finitely dominated and $[C]=0 \in \tilde{K}_{0}(A)$.

(ii) If $\mathscr{F}(C)$ is nonempty it is an affine $\tilde{K}_{1}(A)$-set, with a transitive $\tilde{K}_{1}(A)$-action defined by

$$
\widetilde{K}_{1}(A) \times \mathscr{F}(C) \rightarrow \mathscr{F}(C) ; \quad(\tau(D),(F, \phi)) \mapsto(F \oplus D, \phi \oplus 0)
$$

with $\tau(D) \in \widetilde{K}_{1}(A)$ the reduced torsion of a contractible finite chain complex $D$ over $A$. $A$ choice of base point $\left(F_{0}, \phi_{0}\right) \in \mathscr{F}(C)$ determines an Abelian group structure on $\mathscr{F}(C)$ 
with an isomorphism

$$
\mathscr{F}(C) \rightarrow \tilde{K}_{1}(A) ; \quad(F, \phi) \mapsto \tau\left(\phi^{-1} \phi_{0}: F_{0} \rightarrow F\right) .
$$

Proof. Immediate from Proposition 1.1 (i).

Given a chain equivalence $f: C \rightarrow D$ of chain complexes over $A$ with finite structures $(F, \phi) \in \mathscr{F}(C),(G, \theta) \in \mathscr{F}(D)$ define the reduced torsion

$$
\tau(f)=\tau\left(\theta^{-1} f \phi: F \stackrel{\phi}{\longrightarrow} C \stackrel{f}{\longrightarrow} D \stackrel{\theta^{-1}}{\longrightarrow} G\right) \in \tilde{K}_{1}(A)
$$

This evidently depends on the choices of finite structures as well as $f$, with the reduced torsion $\tau^{\prime}(f) \in \tilde{K}_{1}(A)$ determined by different choices $\left(F^{\prime}, \phi^{\prime}\right) \in \mathscr{F}(C)$, $\left(G^{\prime}, \theta^{\prime}\right) \in \mathscr{F}(D)$ such that

$$
\tau^{\prime}(f)-\tau(f)=\tau\left(\theta^{-1} \theta^{\prime}: G^{\prime} \rightarrow G\right)-\tau\left(\phi^{-1} \phi^{\prime}: F^{\prime} \rightarrow F\right) \in \widetilde{K}_{1}(A),
$$

by the logarithmic property of reduced torsion.

A f.g. free $A$-module $M$ is even if $\operatorname{rank}_{A}(M) \equiv 0(\bmod 2)$. Thus, if either $M$ or $N$ is even $\varepsilon(M, N)=0 \in \mathbb{Z}_{2}$.

A finite chain complex $C$ over $A$ is even if each $C_{r}(r \geqslant 0)$ is an even f.g. free $A$ module. Thus, if either $C$ or $D$ is even $\beta(C, D)=0 \in \mathbb{Z}_{2}$.

(Let $\mathscr{C}^{e}(A)$ be the additive category of even finite chain complexes over $A$ and chain homotopy classes of chain maps. The torsion function

$$
\tau \text { : iso }\left(\mathscr{C}^{e}(A)\right) \rightarrow K_{1}(A) ; \quad f \mapsto \tau(f)=\tau(C(f))
$$

is both logarithmic $(\tau(g f)=\tau(f)+\tau(g))$ and additive $\left(\tau\left(f \oplus f^{\prime}\right)=\tau(f)+\tau\left(f^{\prime}\right)\right)$, agreeing with the torsion $\tau: \operatorname{iso}\left(\mathscr{C}^{r}(A)\right) \rightarrow K_{1}(A) ; f \mapsto \tau(f)$ defined above for the additive category $\mathscr{C}^{r}(A)$ of round finite chain complexes over $A$ and chain homotopy classes of chain maps.)

A round finite structure on a chain complex $C$ over $A$ is an equivalence class of pairs

(round finite chain complex $C$ over $A$, chain equivalence $\phi: F \rightarrow C$ )

under the equivalence relation

$$
(F, \phi) \sim\left(F^{\prime}, \phi^{\prime}\right) \quad \text { if } \tau\left(\phi^{\prime-1} \phi: F \rightarrow F^{\prime}\right)=0 \in K_{1}(A)
$$

The round finite structure set $\mathscr{F}^{r}(C)$ of a chain complex $C$ over $A$ is the set (possibly empty) of round finite structures on $C$.

PROPOSITION 1.6. (i) The round finite structure set $\mathscr{F}^{r}(C)$ is nonempty if and only if $C$ is finitely dominated and $[C]=0 \in K_{0}(A)$.

(ii) If $\mathscr{F}^{r}(C)$ is nonempty it is an affine $K_{1}(A)$-set, with a transitive $K_{1}(A)$-action 
defined by

$$
K_{1}(A) \times \mathscr{F}^{r}(C) \rightarrow \mathscr{F}^{r}(C) ; \quad(\tau(D),(F, \phi)) \mapsto(F \oplus D, \phi \oplus 0)
$$

with $\tau(D) \in K_{1}(A)$ the torsion of a contractible even finite chain complex $D$ over $A$. $A$ choice of base point $\left(F_{0}, \phi_{0}\right) \in \mathscr{F} r(C)$ determines an Abelian group structure on $\mathscr{F}^{r}(C)$ with an isomorphism

$$
\mathscr{F}^{r}(C) \rightarrow K_{1}(A) ; \quad(F, \phi) \mapsto \tau\left(\phi^{-1} \phi_{0}: F_{0} \rightarrow F\right) .
$$

Proof. By analogy with Proposition 1.5.

Given a chain equivalence $f: C \rightarrow D$ of chain complexes over $A$ with round finite structures $(F, \phi) \in \mathscr{F}^{r}(C),(G, \theta) \in \bar{F}^{r}(D)$ define the torsion

$$
\tau(f)=\tau\left(\theta^{-1} f \phi: F \stackrel{\phi}{\longrightarrow} C \stackrel{f}{\longrightarrow} D \stackrel{\theta^{-1}}{\longrightarrow} G\right) \in K_{1}(A) .
$$

This evidently depends on the choices of round finite structures as well as $f$, with the torsion $\tau^{\prime}(f) \in K_{1}(A)$ determined by different choices $\left(F^{\prime}, \phi^{\prime}\right) \in \mathscr{F}^{r}(C),\left(G^{\prime}, \theta^{\prime}\right) \in \mathscr{F}^{r}(D)$ such that

$$
\tau^{\prime}(f)-\tau(f)=\tau\left(\theta^{-1} \theta^{\prime}: G^{\prime} \rightarrow G\right)-\tau\left(\phi^{-1} \phi^{\prime}: F^{\prime} \rightarrow F\right) \in K_{1}(A)
$$

by the logarithmic property of torsion.

The absolute $K_{1}$-group $K_{1}(A)$ behaves better under products than the reduced $K_{1}$-group $\tilde{K}_{1}(A)$, so that round finite structures behave better under products than finite structures. In Section 3 below we shall investigate this behaviour in some detail, using the following sharper version of the condition $\chi(C)=0 \in \mathbb{Z}$ for a finite chain complex $C$ to be round.

Given a finite chain complex $C$ over $A$ define the integers $e_{r}(C)=$ $\operatorname{rank}_{A}\left(C_{r}\right)-\operatorname{rank}_{A}\left(C_{r-1}\right)+\cdots+(-)^{r} \operatorname{rank}_{A}\left(C_{0}\right) \in \mathbb{Z}(r \geqslant 0)$, uniquely characterized by

$$
\operatorname{rank}_{A}\left(C_{r}\right)=e_{r}(C)+e_{r-1}(C) \quad\left(r \geqslant 0, e_{-1}(C)=0\right) .
$$

If $C$ is $n$-dimensional, then for $r \geqslant n$

$$
e_{r}(C)=(-)^{r} \chi(C) \in \mathbb{Z}
$$

A finite chain complex $C$ over $A$ is rounded if $e_{r}(C) \geqslant 0(r \geqslant 0)$. If $C$ is $n$ dimensional $e_{n}(C) e_{n+1}(C)=-\chi(C)^{2} \geqslant 0$, so that $\chi(C)=0$ and $C$ is round. However, a round finite chain complex need not be rounded, as is clear from the example

$$
C: \cdots \rightarrow 0 \rightarrow A \rightarrow A \rightarrow 0 .
$$

PROPOSITION 1.7. (i) A finite chain complex $C$ over $A$ is rounded if and only if there is defined a contractible finite chain complex $C_{\Delta}$ over $A$ with the same chain modules $\left\{C_{r} \mid r \geqslant 0\right\}$

$$
C_{\Delta}: \cdots \rightarrow C_{r+1} \stackrel{d_{\Delta}}{\longrightarrow} C_{r} \stackrel{d_{\Delta}}{\longrightarrow} C_{r-1} \rightarrow \cdots \stackrel{d_{\Delta}}{\longrightarrow} C_{0} .
$$


(ii) For any round finite chain complex $C$ over $A$ there exists a contractible finite chain complex $C^{\prime}$ over $A$ such that $C \oplus C^{\prime}$ is rounded and

$$
\tau\left(\left(\begin{array}{l}
1 \\
0
\end{array}\right): C \rightarrow C \oplus C^{\prime}\right)=0 \in K_{1}(A) .
$$

Proof. (i) Given a contraction $\Gamma: 0 \simeq 1: D \rightarrow D$ of a finite chain complex $D$ over $A$ there are defined stably f.g. free $A$-modules

$$
E_{r}=\operatorname{ker}\left(d: D_{r} \rightarrow D_{r-1}\right)=\operatorname{im}\left(d: D_{r+1} \rightarrow D_{r}\right) \quad(r \geqslant 0)
$$

and isomorphisms

$$
f: D_{r} \rightarrow E_{r} \oplus E_{r-1} ; \quad x \mapsto(d \Gamma(x), d(x)) \quad(r \geqslant 0)
$$

such that

$$
f d f^{-1}=\left(\begin{array}{ll}
0 & 1 \\
0 & 0
\end{array}\right): E_{r} \oplus E_{r-1} \rightarrow E_{r-1} \oplus E_{r-2} .
$$

Now

$$
e_{r}(D)=\operatorname{rank}_{A}\left(E_{r}\right) \geqslant 0 \quad(r \geqslant 0),
$$

so that $D$ is rounded.

Thus, if $C$ is such that there exists a contractible finite chain complex $C_{\Delta}$ with the same chain modules

$$
e_{r}(C)=e_{r}\left(C_{\Delta}\right) \geqslant 0 \quad(r \geqslant 0)
$$

and $C$ is rounded.

Conversely, if $C$ is a rounded finite chain complex over $A$ define $d_{\Delta} \in \operatorname{Hom}_{A}\left(C_{r}, C_{r-1}\right)(r \geqslant 1)$ by

$$
\begin{aligned}
& d_{\Delta}\left(k \text { th base element of } C_{r}\right) \\
& \quad=\left\{\begin{array}{l}
0 \in C_{r-1} \quad \text { if } 1 \leqslant k \leqslant e_{r}(C) \\
\left(k-e_{r}(C)\right) \text { th base element } \in C_{r-1} \text { if } e_{r}(C)+1 \leqslant k \leqslant \operatorname{rank}_{A}\left(C_{r}\right) .
\end{array}\right.
\end{aligned}
$$

Then $C_{\Delta}$ is a contractible chain complex, with a chain contraction $\Gamma: 0 \simeq 1: C_{\Delta} \rightarrow C_{\Delta}$ defined by

$\Gamma\left(k\right.$ th base element of $\left.C_{r}\right)$

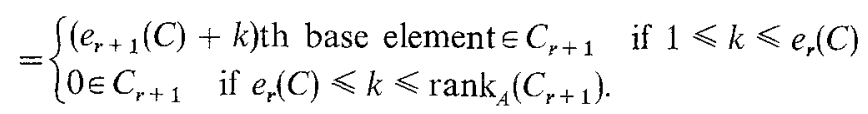

(ii) Let $C$ be $n$-dimensional, and let $\left\{C_{r}^{\prime} \mid r \geqslant 0\right\}$ be a sequence of based f.g. free $A$ modules with the ranks

$$
\operatorname{rank}_{A}\left(C^{\prime}\right)=e_{r}\left(C^{\prime}\right)+e_{r-1}\left(C^{\prime}\right) \quad\left(r \geqslant 0, e_{-1}\left(C^{\prime}\right)=0\right)
$$


determined by the nonnegative integers

$$
e_{r}\left(C^{\prime}\right)=\left\{\begin{array}{l}
\operatorname{rank}_{A}\left(C_{r-1}\right)+\operatorname{rank}_{A}\left(C_{r-3}\right)+\cdots \\
0 \quad \text { if } r>n .
\end{array}\right.
$$

Then $\left\{C_{r} \oplus C_{r}^{\prime} \mid r \geqslant 0\right\}$ is a sequence of based f.g. free $A$-modules such that the ranks

$$
\operatorname{rank}_{A}\left(C_{r} \oplus C_{r}^{\prime}\right)=e_{r}\left(C \oplus C^{\prime}\right)+e_{r-1}\left(C \oplus C^{\prime}\right) \quad(r \geqslant 0)
$$

are determined by the nonnegative integers

$$
\begin{aligned}
e_{r}\left(C \oplus C^{\prime}\right) & =e_{r}(C)+e_{r}\left(C^{\prime}\right) \\
& = \begin{cases}\operatorname{rank}_{A}\left(C_{r}\right)+\operatorname{rank}_{A}\left(C_{r-2}\right)+\cdots & \text { if } r \leqslant n \\
0 & \text { if } r>n .\end{cases}
\end{aligned}
$$

By (i) differentials $\left\{d_{C} \in \operatorname{Hom}_{A}\left(C_{r}^{\prime}, C_{r-1}^{\prime}\right) \mid r \geqslant 0\right\}$ may be chosen such that $C^{\prime}$ is a contractible finite chain complex over $A$, and in particular such that

$$
\tau\left(C^{\prime}\right)=\beta\left(C, C^{\prime}\right) \in K_{1}(A) .
$$

By the sum formula of Proposition 1.2 (ii)

$$
\begin{aligned}
\tau\left(\left(\begin{array}{l}
1 \\
0
\end{array}\right): C \rightarrow C \oplus C^{\prime}\right) & =\tau\left(1 \oplus 0: C \oplus 0 \rightarrow C \oplus C^{\prime}\right) \\
& =\tau(1: C \rightarrow C)+\tau\left(0: 0 \rightarrow C^{\prime}\right)-\beta(C, 0)+\beta\left(C, C^{\prime}\right) \\
& =0 \in K_{1}(A) .
\end{aligned}
$$

\section{Change of Rings}

In the applications we shall be dealing not only with the algebraic $K$-groups $K_{0}(A)$, $K_{1}(A)$ of a single ring $A$, but also with the morphisms of $K$-groups induced by a morphism of rings $f: A \rightarrow B$. As usual, given such a ring morphism regard $B$ as a $(B, A)$-bimodule by

$$
B \times B \times A \rightarrow B ; \quad(b, x, a) \mapsto b x f(a),
$$

so that there is defined a functor

$$
f_{!}:(A \text {-modules }) \rightarrow(B \text {-modules }) ; \quad M \mapsto f_{!} M=B \otimes_{A} M
$$

sending f.g. projective (resp. free) $A$-modules to f.g. projective (resp. free) $B$-modules. Given a finitely dominated (resp. contractible finite) chain complex $C$ over $A$ there is induced a finitely dominated (resp. contractible finite) chain complex $f_{!} C=B \otimes{ }_{A} C$ over $B$, and the induced morphisms of $K$-groups are such that

$$
\begin{array}{ll}
f_{!}: K_{0}(A) \rightarrow K_{0}(B) ; & {[C] \mapsto\left[f_{!} C\right]} \\
f_{!}: K_{1}(A) \rightarrow K_{1}(B) ; & \tau(C) \mapsto \tau\left(f_{!} C\right) .
\end{array}
$$

We shall be particularly concerned with the case in which $f: A \rightarrow B$ is an isomor- 
phism, when it is possible to identify the $B$-module $f_{!} M$ induced by an $A$-module $M$ with the $B$-module defined by the additive group of $M$ with $B$ acting by

$$
B \times f_{!} M \rightarrow f_{!} M ; \quad(b, x) \mapsto f^{-1}(b) x .
$$

For the inner automorphism of a ring $A$

$$
f: A \rightarrow A ; \quad a \mapsto z^{-1} a z
$$

defined by conjugation by a unit $z \in A$ there is defined a natural equivalence of functors

$$
z: 1 \sim f_{!}:(A \text {-modules }) \rightarrow(A \text {-modules }),
$$

with a natural $A$-module isomorphism

$$
z: M \rightarrow f ! M ; \quad x \mapsto z x
$$

for any $A$-module $M$. Thus, for any chain complex $C$ over $A$ there is defined an isomorphism

$$
z: C \rightarrow f_{!} C ; \quad x \mapsto z x .
$$

If $C$ is finitely dominated

$$
f_{1}[C]=\left[f_{!} C\right]=[C] \in K_{0}(A) .
$$

If $C$ is finite then

$$
\begin{aligned}
\tau(z: C \rightarrow f ! C) & =\sum_{r=0}^{\infty}(-)^{r} \tau\left(z: C_{r} \rightarrow f_{!} C_{r}\right) \\
& =\chi(C) \tau(z: A \rightarrow A ; a \mapsto a z) \in K_{1}(A),
\end{aligned}
$$

so that if $C$ is contractible finite

$$
f_{1} \tau(C)=\tau\left(f_{1} C\right)=\tau(C) \in K_{1}(A) .
$$

Thus for an inner automorphism $f: A \rightarrow A$

$$
\begin{aligned}
& f_{!}=1: K_{0}(A) \rightarrow K_{0}(A) . \\
& f_{!}=1: K_{1}(A) \rightarrow K_{1}(A) .
\end{aligned}
$$

A stable isomorphism of f.g. projective $A$-modules [ $\phi]: P \rightarrow Q$ is an equivalence class of isomorphisms $\phi: P \oplus X \rightarrow Q \oplus X$ for f.g. projective $A$-modules $X$, defined exactly as in Section 1 for the additive category of f.g. projective $A$-modules, with

$$
(\phi: P \oplus X \rightarrow Q \oplus X) \sim(\theta: P \oplus Y \rightarrow Q \oplus Y)
$$


if

$$
\begin{gathered}
\tau\left(P \oplus X \oplus Y \stackrel{\phi \oplus 1_{Y}}{\longrightarrow} Q \oplus X \oplus Y \stackrel{1_{Q} \oplus\left(\begin{array}{ll}
0 & 1_{Y} \\
1_{X} & 0
\end{array}\right)}{\longrightarrow} Q \oplus Y \oplus X\right. \\
\left.\stackrel{\theta^{-1} \oplus 1_{X} \longrightarrow}{\longrightarrow} P \oplus Y \oplus X \stackrel{1_{P} \oplus\left(\begin{array}{ll}
0 & 1_{X} \\
1_{Y} & 0
\end{array}\right)}{\longrightarrow} P \oplus X \oplus Y\right) \\
=0 \in K_{1}(A) .
\end{gathered}
$$

Note that f.g. projective $A$-modules $P, P^{\prime}, Q, Q^{\prime}$ are such that

$$
[P]-[Q]=\left[P^{\prime}\right]-\left[Q^{\prime}\right] \in K_{0}(A)
$$

if and only if $P \oplus Q^{\prime}$ is stably isomorphic to $Q \oplus P^{\prime}$.

Define the relative $K_{1}$-group $K_{1}(f)$ of a morphism $f: A \rightarrow B$ of rings to be the Abelian group of equivalence classes of triples $(P, Q,[\phi])$ defined by f.g. projective $A$-modules $P, Q$ and a stable isomorphism $[\phi]: f_{!} P \rightarrow f_{!} Q$ of the induced f.g. projective $B$-modules, under the equivalence relation

$(P, Q,[\phi]) \sim\left(P^{\prime}, Q^{\prime},\left[\phi^{\prime}\right]\right)$ if there exists a stable isomorphism

$[\theta]: P \oplus Q^{\prime} \rightarrow Q \oplus P^{\prime}$ such that

$$
\begin{aligned}
& \tau\left(f_{!} P \oplus f_{!} Q^{\prime} \stackrel{f_{!}[\theta]}{\longrightarrow} f_{1} Q \oplus f_{!} P^{\prime} \stackrel{[\phi]^{-1} \oplus\left[\phi^{\prime}\right]}{\longrightarrow} f_{!} P \oplus f_{!} Q^{\prime}\right) \\
& \quad=0 \in K_{1}(B)
\end{aligned}
$$

with addition by

$$
(P, Q,[\phi])+(R, S,[\psi])=(P \oplus R, Q \oplus S,[\phi] \oplus[\psi]) \in K_{1}(f) .
$$

$K_{1}(f)$ is isomorphic to the relative $K_{1}$-group defined by Bass [1]. Note the logarithmic property

$$
(P, Q,[\phi])+(Q, R,[\psi])=(P, R,[\psi][\phi]) \in K_{1}(f),
$$

so that inverses are given by

$$
-(P, Q,[\phi])=\left(Q, P,[\phi]^{-1}\right) \in K_{1}(f) .
$$

PROPOSITION 2.1. The relative $K_{1}$-group $K_{1}(f)$ fits into an exact sequence

$$
K_{1}(A) \stackrel{f_{!}}{\longrightarrow} K_{1}(B) \stackrel{j}{\longrightarrow} K_{1}(f) \stackrel{\hat{o}}{\longrightarrow} K_{0}(A) \stackrel{f_{!}}{\longrightarrow} K_{0}(B)
$$

with

$$
\begin{array}{ll}
j: K_{1}(B) \rightarrow K_{1}(f) ; & \tau(\phi: X \rightarrow X) \mapsto(0,0,[\phi]) \\
\partial: K_{1}(f) \rightarrow K_{0}(A) ; & (P, Q,[\phi]) \mapsto[Q]-[P] .
\end{array}
$$


Proof. Trivial.

Given finitely dominated chain complexes $C, D$ over $A$ and a chain equivalence of the induced chain complexes over $B$

$$
\zeta: f_{!} C \rightarrow f_{!} D
$$

there is defined an element $(C, D, \zeta) \in K_{1}(f)$ such that

$$
\partial(C, D, \zeta)=[D]-[C] \in K_{0}(A)
$$

as follows. Choose chain equivalences $\theta: C \rightarrow P, \psi: D \rightarrow Q$ to bounded f.g. projective chain complexes $P, Q$ over $A$ and define a chain equivalence of the induced chain complexes over $B$

$$
\phi=\left(f_{!} \psi\right) \zeta\left(f_{!} \theta^{-1}\right): f_{!} P \stackrel{f_{1} \theta^{-1}}{\longrightarrow} f_{!} C \stackrel{\zeta}{\longrightarrow} f_{!} D \stackrel{f_{!} \psi}{\longrightarrow} f_{!} Q .
$$

Using any chain contraction $\Gamma: 0 \simeq 1: C(\phi) \rightarrow C(\phi)$ and the isomorphism of f.g. projective $B$-modules

$$
d+\Gamma: C(\phi)_{\text {odd }}=f_{!} P_{\text {even }} \oplus f_{!} Q_{\text {odd }} \rightarrow C(\phi)_{\text {even }}=f_{!} P_{\text {odd }} \oplus f_{!} Q_{\text {even }}
$$

define an element

$$
(C, D, \zeta)=\left(P_{\text {even }} \oplus Q_{\text {odd }}, P_{\text {odd }} \oplus Q_{\text {even }}, d+\Gamma\right) \in K_{1}(f)
$$

which is independent of the choices of $\theta, \psi, \Gamma$. The definition of $(C, D, \zeta) \in K_{1}(f)$ is a mild generalization of a construction of Smith [20]. In Section 4 below we shall use the construction to define a relative $K_{1}$-theory invariant $(X, Y, \zeta) \in K_{1}(f)$ for a map $\zeta: X \rightarrow Y$ of finitely dominated $\mathrm{CW}$ complexes which is a $B$-homology equivalence, for some morphism of rings $f: A=\mathbb{Z}\left[\pi_{1}(Y)\right] \rightarrow B$. (More generally, there is defined an invariant $(C, D, \xi) \in K_{1}(f)$ for any chain equivalence

$$
\zeta: f_{!} C \oplus E \rightarrow f_{!} D \oplus F
$$

with $C, D$ finitely dominated chain complexes over $A$ and $E, F$ round finite chain complexes over $B$. The element is such that

$$
(C, D, \zeta)=[D]-[C] \in K_{0}(A),
$$

and

$$
j: K_{1}(B) \rightarrow K_{1}(f) ; \quad \tau(\zeta: E \rightarrow F) \mapsto(0,0, \zeta) .
$$

We need only consider $(C, D, \zeta) \in K_{1}(f)$ for $E=0, F=0$ here.)

Given two ring morphisms $f, g: A \rightarrow B$ define the relative $K_{1}$-group $K_{1}(f, g)$ to be the Abelian group with one generator $(P,[\phi])$ for each f.g. projective $A$-module $P$ with a stable isomorphism $[\phi]: g_{!} P \rightarrow f_{1} P$ of the induced f.g. projective $B$-modules, 
subject to the relations

$$
\begin{aligned}
(P,[\phi])= & \left(P^{\prime},\left[\phi^{\prime}\right]\right) \text { if there exists a stable isomorphism } \\
& {[\theta]: P \rightarrow P^{\prime} \quad \text { such that } } \\
& \tau\left(g_{!}[\theta]^{-1}[\phi]^{-1} f_{!}[\theta][\phi]: g_{!} P \rightarrow f_{!} P \rightarrow f_{!} P^{\prime} \rightarrow g_{!} P^{\prime} \rightarrow g_{!} P\right) \\
= & 0 \in K_{1}(B), \\
(P,[\phi])+ & \left(P^{\prime},[\phi]\right)=\left(P \oplus P^{\prime},[\phi] \oplus\left[\phi^{\prime}\right]\right) \in K_{1}(f, g) .
\end{aligned}
$$

PROPOSITION 2.2. The relative $K_{1}$-group $K_{1}(f, g)$ fits into an exact sequence

$$
K_{1}(A) \stackrel{f_{1}-g_{1}}{\longrightarrow} K_{1}(B) \stackrel{j}{\longrightarrow} K_{1}(f, g) \stackrel{\partial}{\longrightarrow} K_{0}(A) \stackrel{f_{1}-g_{1}}{\longrightarrow} K_{0}(B)
$$

with

$$
\begin{aligned}
& j: K_{1}(B) \rightarrow K_{1}(f, g) ; \quad \tau\left(\phi: B^{n} \rightarrow B^{n}\right) \mapsto\left(A^{n},[\phi]\right)-\left(A^{n},[1]\right) \\
& \partial: K_{1}(f, g) \rightarrow K_{0}(A) ; \quad(P,[\phi]) \mapsto[P] .
\end{aligned}
$$

Proof. Define $K_{1}^{\prime}(f, g)$ to be the Abelian group of equivalence classes of triples ( $P, Q,[\phi])$ consisting of f.g. projective $A$-modules $P, Q$ and a stable isomorphism of f.g. projective $B$-modules

$$
[\phi]: g_{!} P \oplus f_{1} Q \rightarrow f_{!} P \oplus g_{!} Q
$$

under the equivalence relation

$$
\begin{aligned}
(P, Q,[\phi]) \sim & \left(P^{\prime}, Q^{\prime},\left[\phi^{\prime}\right]\right) \quad \text { if there exists a stable isomorphism } \\
& {[\theta]: P \oplus Q^{\prime} \rightarrow P^{\prime} \oplus Q \text { such that } } \\
& \tau\left(\left(f_{!}[\theta]^{-1} \oplus g_{!}[\theta]\right)\left([\phi] \oplus[\phi]^{-1}\right):\right. \\
& g_{!} P \oplus f_{!} Q \oplus g_{!} P^{\prime} \oplus f_{!} Q^{\prime} \rightarrow f_{!} P \oplus g_{!} Q \oplus f_{!} P^{\prime} \oplus g_{!} Q^{\prime} \\
& \left.\rightarrow g_{!} P \oplus f_{!} Q \oplus g_{!} P^{\prime} \oplus f_{!} Q^{\prime}\right) \\
= & 0 \in K_{1}(B) .
\end{aligned}
$$

It follows from the logarithmic property

$$
(P, Q,[\phi]) \oplus(Q, R,[\psi])=(P, R,[\psi][\phi]) \in K_{1}^{\prime}(f, g)
$$

that inverses are given by

$$
-(P, Q,[\phi])=\left(Q, P,[\phi]^{-1}\right) \in K_{1}^{\prime}(f, g) .
$$

Now $K_{1}^{\prime}(f, g)$ fits into an exact sequence

$$
K_{1}(A) \stackrel{f_{1}-g_{1}}{\longrightarrow} K_{1}(B) \stackrel{j^{\prime}}{\longrightarrow} K_{1}^{\prime}(f, g) \stackrel{\partial^{\prime}}{\longrightarrow} K_{0}(A) \stackrel{f_{1}-g_{!}}{\longrightarrow} K_{0}(B)
$$

with

$$
\begin{array}{ll}
j^{\prime}: K_{1}(B) \rightarrow K_{1}^{\prime}(f, g) ; \quad \tau\left(\phi: B^{n} \rightarrow B^{n}\right) \mapsto(0,0,[\phi]) \\
\partial^{\prime}: K_{1}^{\prime}(f, g) \rightarrow K_{0}(A) ; \quad(P, Q,[\phi]) \mapsto[P]-[Q],
\end{array}
$$


and there is defined an isomorphism of Abelian groups

$$
h: K_{1}(f, g) \rightarrow K_{1}^{\prime}(f, g) ; \quad(P,[\phi]) \mapsto(P, 0,[\phi])
$$

with inverse

$$
\begin{aligned}
& h^{-1}: K_{1}^{\prime}(f, g) \rightarrow K_{1}(f, g) ; \\
&(P, Q,[\phi]) \mapsto(P \oplus-Q,[\phi])-(Q \oplus-Q,[1]) \\
& \quad\left(\text { for any }-Q \text { such that } Q \oplus-Q=A^{n}\right)
\end{aligned}
$$

such that $h j=j^{\prime}, \partial^{\prime} h=\partial$.

In the applications we shall use the isomorphism $h: K_{1}(f, g) \rightarrow K_{1}^{\prime}(f, g)$ as an identification, representing elements of $K_{1}(f, g)$ both as pairs $\left(P,[\phi]: g_{!} P \rightarrow f_{!} P\right)$ and as triples $\left(P, Q,[\phi]: g_{!} P \oplus f_{!} Q \rightarrow f_{!} P \oplus g_{!} Q\right)$.

(Given ring morphisms $f_{1}: A \rightarrow B_{1}, f_{2}: A \rightarrow B_{2}$ define ring morphisms from $A$ to the product ring $B_{1} \times B_{2}$

$$
\begin{aligned}
f: A \rightarrow B_{1} \times B_{2} ; & a \mapsto\left(f_{1}(a), 0\right), \\
g: A \rightarrow B_{1} \times B_{2} ; & a \mapsto\left(0, f_{2}(a)\right) .
\end{aligned}
$$

For such $f, g$ the exact sequence of Proposition 2.2 can be written as

$$
\begin{gathered}
K_{1}(A) \stackrel{\left(\begin{array}{l}
f_{1 !} \\
-f_{2 !}
\end{array}\right)}{\longrightarrow} K_{1}\left(B_{1}\right) \oplus K_{1}\left(B_{2}\right) \stackrel{j}{\longrightarrow} K_{1}(f, g) \\
\stackrel{\partial}{\longrightarrow} K_{0}(A) \stackrel{\left(\begin{array}{c}
f_{1 !} \\
-f_{2 !}
\end{array}\right)}{\longrightarrow} K_{0}\left(B_{1}\right) \oplus K_{0}\left(B_{2}\right)
\end{gathered}
$$

and $K_{1}(f, g)$ is isomorphic to the relative $K_{1}$-group defined by Casson [3].)

Given a finitely dominated chain complex $C$ over $A$ and a chain equivalence of the induced chain complexes over $B$ for some ring morphisms $f, g: A \rightarrow B$

$$
\zeta: g_{!} C \rightarrow f_{!} C
$$

there is defined an element $(C, \zeta) \in K_{1}(f, g)$ such that

$$
\partial(C, \zeta)=[C] \in K_{0}(A)
$$

as follows. Choose a chain equivalence $\psi: C \rightarrow P$ to a bounded f.g. projective chain complex $P$ over $A$ and define a chain equivalence of bounded f.g. projective chain complexes over $B$

$$
\phi=\left(f_{!} \psi\right) \zeta\left(g_{!} \psi^{-1}\right): g_{!} P \stackrel{g_{!} \psi^{-1}}{\longrightarrow} g_{!} C \stackrel{\zeta}{\longrightarrow} f_{!} C \stackrel{f_{!} \psi}{\longrightarrow} f_{!} P .
$$

Using any chain contraction $\Gamma: 0 \simeq 1: C(\phi) \rightarrow C(\phi)$ and the isomorphism of f.g. 
projective $B$-modules

$$
d+\Gamma: C(\phi)_{\text {odd }}=g_{!} P_{\text {even }} \oplus f ! P_{\text {odd }} \rightarrow C(\phi)_{\text {even }}=f_{!} P_{\text {odd }} \oplus g_{!} P_{\text {even }}
$$

define an element

$$
(C, \zeta)=\left(P_{\text {even }}, P_{\text {odd }}, d+\Gamma\right) \in K_{1}(f, g)
$$

which is independent of the choices of $\psi, \Gamma$. In Section 5 below we shall use the construction to define an invariant $(X, \zeta) \in K_{1}\left(1_{A}, \alpha\right)$ for any self-homotopy equivalence $\zeta: X \rightarrow X$ of a finitely dominated CW complex $X$, with $A=\mathbb{Z}\left[\pi_{1}(X)\right]$ and $\alpha: A \rightarrow A$ the automorphism induced by $\zeta_{*}: \pi_{1}(X) \rightarrow \pi_{1}(X)$. (More generally, there is defined an invariant $(C, D, \zeta) \in K_{1}(f, g)$ for any chain equivalence

$$
\zeta: g_{!} C \oplus f_{!} D \oplus E \rightarrow f_{!} C \oplus g_{!} D \oplus F
$$

with $C, D$ finitely dominated chain complexes $C, D$ over $A$ and $E, F$ round finite chain complexes over $B$. The element is such that

$$
\partial(C, D, \zeta)=[C]-[D] \in K_{0}(A)
$$

and

$$
j: K_{1}(B) \rightarrow K_{1}(f, g) ; \tau(\zeta: E \rightarrow F) \mapsto(0,0, \zeta)
$$

We need only consider the case $D=0, E=0, \quad F=0$ here, with $\left.(C, 0, \zeta)=(C, \zeta) \in K_{1}(f, g)\right)$.

\section{Products in $K$-Theory}

Given rings $A, B$ let $A \otimes B, B \otimes A$ be the product rings, where the tensor product is taken over $\mathbb{Z}$. The transposition isomorphisms

$$
\begin{array}{ll}
T: B \otimes A \rightarrow A \otimes B ; & b \otimes a \mapsto a \otimes b \\
U: A \otimes B \rightarrow B \otimes A ; & a \otimes b \mapsto b \otimes a
\end{array}
$$

are inverse to each other.

The product of an $A$-module $M$ and a $B$-module $N$ is an $A \otimes B$-module $M \otimes N$, with $A \otimes B$ acting by

$$
A \otimes B \times M \otimes N \rightarrow M \otimes N ; \quad(a \otimes b, x \otimes y) \mapsto a x \otimes b y,
$$

and the $B \otimes A$-module $N \otimes M$ is defined similarly. If $M$ is a f.g. projective $A$-module and $N$ is a f.g. projective $B$-module then $M \otimes N$ is a f.g. projective $A \otimes B$-module. If $M$ and $N$ are f.g. free then so is $M \otimes N$, and

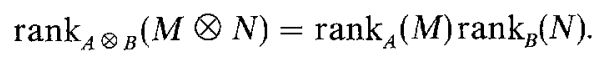

In dealing with based f.g. free modules we adopt the convention that a base $\left\{x_{i} \mid 1 \leqslant i \leqslant m\right\}$ for $M$ and a base $\left\{y_{j} \mid 1 \leqslant j \leqslant n\right\}$ for $N$ determine the base 
$\left\{z_{k} \mid 1 \leqslant k \leqslant m n\right\}$ for $M \otimes N$ defined by

$$
z_{k}=x_{i} \otimes y_{j} \quad \text { if } k=i+m(j-1)
$$

so that

$$
\left\{z_{1}, z_{2}, \ldots, z_{m n}\right\}=\left\{x_{1} \otimes y_{1}, x_{2} \otimes y_{1}, \ldots, x_{m} \otimes y_{1}, x_{1} \otimes y_{2}, \ldots, x_{m} \otimes y_{n}\right\} .
$$

The isomorphism of based f.g. free $A \otimes B$-modules

$M \otimes N \rightarrow T_{!}(N \otimes M) ; \quad x \otimes y \mapsto y \otimes x$

has torsion

$$
\begin{aligned}
& \tau\left(M \otimes N \rightarrow T_{!}(N \otimes M)\right)=\frac{1}{4} m(m-1) n(n-1) \tau(-1: A \otimes B \rightarrow A \otimes B) \\
& \in K_{1}(A \otimes B),
\end{aligned}
$$

the sign of the permutation

$$
\begin{aligned}
& \{1,2, \ldots, m n\} \rightarrow\{1,2, \ldots, m n\} ; \\
& \quad k=i+m(j-1) \mapsto k^{\prime}=j+n(i-1)(1 \leqslant i \leqslant m, 1 \leqslant j \leqslant n) .
\end{aligned}
$$

Furthermore, for based f.g. free $A$-modules $M, M_{1}, M_{2}$ and based f.g. free $B$-modules $N, N_{1}, N_{2}$ the evident isomorphisms of based f.g. free $A \otimes B$-modules have torsions

$$
\begin{aligned}
& \tau\left(M \otimes\left(N_{1} \oplus N_{2}\right) \rightarrow\left(M \otimes N_{1}\right) \oplus\left(M \otimes N_{2}\right)\right)=0 \in K_{1}(A \otimes B) \\
& \tau\left(\left(M_{1} \oplus M_{2}\right) \otimes N \rightarrow\left(M_{1} \otimes N\right) \oplus\left(M_{2} \otimes N\right)\right) \\
& \quad=\frac{1}{2} m_{1} m_{2} n(n-1) \tau(-1: A \otimes B \rightarrow A \otimes B) \in K_{1}(A \otimes B)
\end{aligned}
$$

with $m_{1}=\operatorname{rank}_{A}\left(M_{1}\right), m_{2}=\operatorname{rank}_{A}\left(M_{2}\right), n=\operatorname{rank}_{B}(N)$. The sign is obtained by considering the commutative diagram of isomorphisms

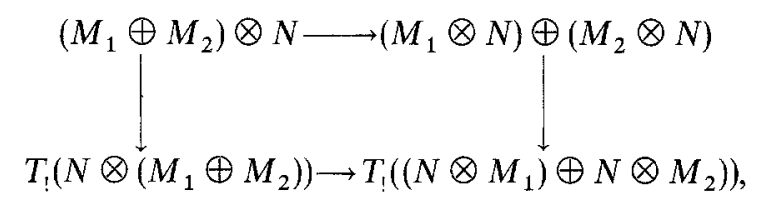

and noting that

$$
\begin{aligned}
\frac{1}{4}\left(m_{1}\right. & \left.+m_{2}\right)\left(m_{1}+m_{2}-1\right) n(n-1)- \\
& \quad-\frac{1}{4} m_{1}\left(m_{1}-1\right) n(n-1)-\frac{1}{4} m_{2}\left(m_{2}-1\right) n(n-1) \\
= & \frac{1}{2} m_{1} m_{2} n(n-1) .
\end{aligned}
$$

The product operation on modules is functorial, and as usual there are defined products in the algebraic $K$-groups

$$
\begin{aligned}
& K_{0}(A) \otimes K_{0}(B) \rightarrow K_{0}(A \otimes B) ; \quad[P] \otimes[Q] \mapsto[P \otimes Q] \\
& K_{1}(A) \otimes K_{0}(B) \rightarrow K_{1}(A \otimes B) ; \\
& \tau(f: P \rightarrow P) \otimes[Q] \mapsto \tau(f \otimes 1: P \otimes Q \rightarrow P \otimes Q) \\
& K_{0}(A) \otimes K_{1}(B) \rightarrow K_{1}(A \otimes B) ; \\
& {[P] \otimes \tau(g: Q \rightarrow Q) \mapsto \tau(1 \otimes g: P \otimes Q \rightarrow P \otimes Q)}
\end{aligned}
$$


with $P$ a f.g. projective $A$-module, $Q$ a f.g. projective $B$-module, and $f \in \operatorname{Hom}_{A}(P, P)$, $g \in \operatorname{Hom}_{B}(Q, Q)$ automorphisms.

The product of an $A$-module chain complex $C$ and a $B$-module chain complex $D$ is the $A \otimes B$-module chain complex $C \otimes D$ defined by

$$
\begin{aligned}
d_{C \otimes D}:(C \otimes D)_{r}= & \Sigma_{s=-\infty}^{\infty} C_{s} \otimes D_{r-s} \rightarrow(C \otimes D)_{r-1} ; \\
& x \otimes y \mapsto x \otimes d_{D}(y)+(-)^{r-s} d_{C}(x) \otimes y .
\end{aligned}
$$

If $C$ and $D$ are finitely dominated, then so is $C \otimes D$, and if either $C$ or $D$ is contractible then so is $C \otimes D$. If $C$ and $D$ are finite, then so is $C \otimes D$, as in $D \otimes C$, and the transposition isomorphism of finite chain complexes over $A \otimes B$

$C \otimes D \rightarrow T_{!}(D \otimes C) ; \quad x \otimes y \mapsto(-)^{s t} y \otimes x \quad\left(x \in C_{s}, y \in D_{t}\right)$

has torsion

$$
\begin{aligned}
& \tau\left(C \otimes D \rightarrow T_{1}(D \otimes C)\right) \\
& \quad=\zeta(C, D) \tau(-1: A \otimes B \rightarrow A \otimes B) \in K_{1}(A \otimes B),
\end{aligned}
$$

where

$$
\begin{aligned}
\zeta(C, D)= & v(C) v(D)+\chi_{\text {odd }}(C) \chi_{\text {odd }}(D)+ \\
& +\sum_{r=0}^{\infty} \Sigma_{0 \leqslant s<t \leqslant r} c_{s} c_{t} d_{r-s} d_{r-t} \in \mathbb{Z}_{2},
\end{aligned}
$$

with

$$
\begin{aligned}
& c_{s}=\operatorname{rank}_{A}\left(C_{s}\right), \quad d_{t}=\operatorname{rank}_{B}\left(D_{t}\right), \\
& v(C)=\sum_{s=0}^{\infty} \frac{1}{2} c_{s}\left(c_{s}-1\right), \\
& \chi_{\text {odd }}(C)=\sum_{i=0}^{\infty} c_{2 i+1} \in \mathbb{Z}_{2} .
\end{aligned}
$$

(Further below we shall also use $\chi_{\text {even }}(C)=\Sigma_{i=0}^{\infty} c_{2 i} \in \mathbb{Z}_{2}$.) If $C, C^{\prime}$ are finite chain complexes over $A$ and $D, D^{\prime}$ are finite chain complexes over $B$ the rearrangement isomorphisms have torsions

$$
\begin{aligned}
& \tau\left(C \otimes\left(D \oplus D^{\prime}\right) \rightarrow(C \otimes D) \oplus\left(C \otimes D^{\prime}\right)\right) \\
& \quad=\lambda\left(C, D, D^{\prime}\right) \tau(-1: A \otimes B \rightarrow A \otimes B) \in K_{1}(A \otimes B) \\
& \tau\left(\left(C \oplus C^{\prime}\right) \otimes D \rightarrow(C \otimes D) \oplus\left(C \otimes D^{\prime}\right)\right) \\
& \quad=\mu\left(C, C^{\prime}, D\right) \tau(-1: A \otimes B \rightarrow A \otimes B) \in K_{1}(A \otimes B)
\end{aligned}
$$

with $\lambda, \mu$ defined by

$$
\begin{aligned}
& \lambda\left(C, D, D^{\prime}\right)=\left(\sum_{s=0}^{\infty} c_{s} c_{s+1}\right)\left(\sum_{t=0}^{\infty} d_{t} d_{t+1}^{\prime}\right), \\
& \mu\left(C, C^{\prime}, D\right)=\lambda\left(D, C, C^{\prime}\right)+\varepsilon\left(C \oplus C^{\prime}, D\right)+\varepsilon(C, D)+\varepsilon\left(C^{\prime}, D\right) \in \mathbb{Z}_{2} .
\end{aligned}
$$

For any finite chain complex $C$ over $A$ and any chain map $g: D \rightarrow D^{\prime}$ of finite chain complexes over $B$, the rearrangement isomorphism $C\left(1 \otimes g: C \otimes D \rightarrow C \otimes D^{\prime}\right) \rightarrow$ 
$C \otimes C\left(g: D \rightarrow D^{\prime}\right)$ has torsion

$$
\begin{aligned}
& \tau(C(1 \otimes g) \rightarrow C \otimes C(g)) \\
& \quad=\lambda\left(D^{\prime}, S D, C\right) \tau(-1: A \otimes B \rightarrow A \otimes B) \in K_{1}(A \otimes B) .
\end{aligned}
$$

For any chain map $f: C \rightarrow C^{\prime}$ of finite chain complexes over $A$ and any finite chain complex $D$ over $B$ the rearrangement isomorphism $C\left(f \otimes 1: C \otimes D \rightarrow C^{\prime} \otimes D\right) \rightarrow$ $\left.C(f: C) \rightarrow C^{\prime}\right) \otimes D$ has torsion

$$
\begin{aligned}
& \tau(C(f \otimes 1) \rightarrow C(f) \otimes D) \\
& \quad=\mu\left(C^{\prime}, S C, D\right) \tau(-1: A \otimes B \rightarrow A \otimes B) \in K_{1}(A \otimes B) .
\end{aligned}
$$

PROPOSITION 3.1. (i) The projective class of the product $C \otimes D$ of a finitely dominated chain complex $C$ over $A$ and a finitely dominated chain complex $D$ over $B$ is given by

$$
[C \otimes D]=[C] \otimes[D] \in K_{0}(A \otimes B) .
$$

(ii) The torsion of the product $C \otimes D$ of a contractible finite chain complex $C$ over $A$ and a finite chain complex $D$ over $B$ is given by

$$
\tau(C \otimes D)=\tau(C) \otimes[D]+\eta(C, D) \tau(-1: A \otimes B \rightarrow A \otimes B) \in K_{1}(A \otimes B)
$$

where $[D]=\chi(D) \in K_{0}(B)$ and $\eta$ is defined by

$$
\begin{aligned}
\eta(C, D)= & \beta(C, C) v(D)+\Sigma_{i>j} \beta\left(C \otimes S^{i} D_{i}, C \otimes S^{j} D_{j}\right)+ \\
& +\chi_{\text {odd }}(C) \chi_{\text {odd }}(D) \in \mathbb{Z}_{2} .
\end{aligned}
$$

(iii) The torsion of the product $C \otimes D$ of a finite chain complex $C$ over $A$ and $a$ contractible finite chain complex $D$ over $B$ is given by

$$
\begin{aligned}
\tau(C \otimes D)= & {[C] \otimes \tau(D)+(\eta(D, C)+} \\
& +\zeta(D, C)) \tau(-1: A \otimes B \rightarrow A \otimes B) \in K_{1}(A \otimes B),
\end{aligned}
$$

where $[C]=\chi(C) \in K_{0}(A)$. If $C$ is even the sign term vanishes and

$$
\tau(C \otimes D)=[C] \otimes \tau(D) \in K_{1}(A \otimes B) .
$$

(iv) The reduced torsion of the product $f \otimes g: C \otimes D \rightarrow C^{\prime} \otimes D^{\prime}$ of a chain equivalence $f: C \rightarrow C^{\prime}$ of finite chain complexes over $A$ and a chain equivalence $g: D \rightarrow D^{\prime}$ of finite chain complexes over $B$ is given by

$$
\tau(f \otimes g)=[C] \otimes \tau(g)+\tau(f) \otimes[D] \in \tilde{K}_{1}(A \otimes B),
$$

where

$$
[C]=\chi(C) \in \mathbb{Z} \subset K_{0}(A), \quad[D]=\chi(D) \in \mathbb{Z} \subset K_{0}(B) .
$$

Proof. (i) By the chain homotopy invariance of the projective class it may be assumed that $C$ and $D$ are bounded positive complexes of f.g. projective modules, in 
which case so is $C \otimes D$ and

$$
\begin{aligned}
{[C \otimes D] } & =\Sigma_{r=0}^{\infty}(-)^{r}\left[(C \otimes D)_{r}\right] \\
& =\Sigma_{r=0}^{\infty} \Sigma_{s+t=r}(-)^{s+t}\left[C_{s} \otimes D_{t}\right] \\
& =\Sigma_{r=0}^{\infty} \Sigma_{s+t=r}(-)^{s+t}\left[C_{s} \otimes\left[D_{t}\right]\right. \\
& =\left(\sum_{s=0}^{\infty}(-)^{s}\left[C_{s}\right] \otimes\left(\Sigma_{t=0}^{\infty}(-)^{t}\left[D_{t}\right]\right)\right. \\
& =[C] \otimes[D] \in K_{0}(A \otimes B) .
\end{aligned}
$$

(ii) If $D$ is 0 -dimensional, then by definition

$$
\tau(C \otimes D)=\tau\left((d+\Gamma) \otimes 1:(C \otimes D)_{\text {odd }} \rightarrow(C \otimes D)_{\text {even }}\right) \in K_{1}(A \otimes B),
$$

for any chain contraction $\Gamma: 0 \simeq 1: C \rightarrow C$. The rearrangement isomorphisms have torsions

$$
\begin{aligned}
& \tau\left((C \otimes D)_{\text {odd }} \rightarrow C_{\text {odd }} \otimes D_{0}\right) \\
& \quad=\left(\Sigma_{i>j} c_{2 i+1} c_{2 j+1}\right) \frac{1}{2} d_{0}\left(d_{0}-1\right) \tau(-1: A \otimes B \rightarrow A \otimes B), \\
& \tau\left((C \otimes D)_{\text {even }} \rightarrow C_{\text {even }} \otimes D_{0}\right) \\
& \quad=\left(\Sigma_{i>j} c_{2 i} c_{2 j}\right) \frac{1}{2} d_{0}\left(d_{0}-1\right) \tau(-1: A \otimes B \rightarrow A \otimes B) \in K_{1}(A \otimes B)
\end{aligned}
$$

and

$$
(d+\Gamma) \otimes 1:(C \otimes D)_{\mathrm{odd}} \rightarrow C_{\text {odd }} \otimes D_{0}-\stackrel{(d+\Gamma) \otimes 1}{\rightarrow} C_{\text {even }} \otimes D_{0} \rightarrow(C \otimes D)_{\text {even }},
$$

so that

$$
\begin{aligned}
\tau(C \otimes D)= & \tau\left((C \otimes D)_{\text {odd }} \rightarrow C_{\text {odd }} \otimes D_{0}\right)+\tau\left((d+\Gamma) \otimes 1: C_{\text {odd }} \otimes D_{0} \rightarrow C_{\text {even }} \otimes D_{0}\right)- \\
& -\tau\left((C \otimes D)_{\text {even }} \rightarrow C_{\text {even }} \otimes D_{0}\right) \\
= & \tau(C) \otimes[D]+\beta(C, C) \nu(D) \tau(-1: A \otimes B \rightarrow A \otimes B) \\
= & \tau(C) \otimes[D]+\eta(C, D) \tau(-1: A \otimes B \rightarrow A \otimes B) \in K_{1}(A \otimes B) .
\end{aligned}
$$

Assume inductively that $\tau(C \otimes D)=\tau(C) \otimes[D]+\eta(C, D) \tau(-1)$ if $D$ is of dimension $<n$. If $D$ is $n$-dimensional, let $D^{\prime}$ be the $(n-1)$-skeleton, so that there is defined a short exact sequence of finite chain complexes over $B$

$$
0 \rightarrow D^{\prime} \stackrel{i}{\longrightarrow} D \stackrel{j}{\rightarrow} S^{n} D_{n} \rightarrow 0
$$

with

$$
\left(S^{n} D_{n}\right)_{r}=D_{n} \quad \text { if } r=n,=0 \text { if } r \neq n .
$$

Applying $C \otimes$ - there is obtained a short exact sequence of finite chain complexes over $A \otimes B$

$$
0 \rightarrow C \otimes D^{\prime} \stackrel{1 \otimes i}{\longrightarrow} C \otimes D \stackrel{1 \otimes j}{\longrightarrow} C \otimes S^{n} D_{n} \rightarrow 0
$$

By the sum formula of Proposition 2.3 of [16] and the inductive hypothesis $\tau(C \otimes D)=\tau\left(C \otimes D^{\prime}\right)+\tau\left(C \otimes S^{n} D_{n}\right)+\beta\left(C \otimes D^{\prime}, C \otimes S^{n} D_{n}\right) \tau(-1: A \otimes B \rightarrow A \otimes B)$ 


$$
\begin{aligned}
= & \tau(C) \otimes\left[D^{\prime}\right]+(-)^{n} \tau(C) \otimes\left[D_{n}\right]+ \\
& +\left(\beta\left(C \otimes D^{\prime}, C \otimes S^{n} D_{n}\right)+n \chi_{\text {odd }}(C)+\sum_{m<n} \beta\left(C \otimes S^{m} D_{m}, C \otimes S^{n} D_{n}\right)\right) \tau(-1) \\
= & \tau(C) \otimes[D]+\eta(C, D) \tau(-1: A \otimes B \rightarrow A \otimes B) \in K_{1}(A \otimes B),
\end{aligned}
$$

establishing the inductive step.

(iii) Using the transposition isomorphisms

$$
T: B \otimes A \rightarrow A \otimes B, \quad U: A \otimes B \rightarrow B \otimes A
$$

and the result of (ii) we have

$$
\begin{aligned}
\tau(C \otimes D) & =T_{!} U_{!} \tau(C \otimes D) \\
& =T_{1}(\tau(D \otimes C)+\eta(D, C) \tau(-1: B \otimes A \rightarrow B \otimes A)) \\
& =T_{1}(\tau(D) \otimes[C]+(\zeta(D, C)+\eta(D, C)) \tau(-1: B \otimes A \rightarrow B \otimes A)) \\
& =[C] \otimes \tau(D)+(\zeta(D, C)+\eta(D, C)) \tau(-1: A \otimes B \rightarrow A \otimes B) \in K_{1}(A \otimes B) .
\end{aligned}
$$

LEMMA. For any finite chain complex $C$ over $A$

$$
\beta(C, C)=v(C)+\frac{1}{2} \chi_{\text {even }}(C)\left(\chi_{\text {even }}(C)-1\right)+\frac{1}{2} \chi_{\text {odd }}(C)\left(\chi_{\text {odd }}(C)-1\right) \in \mathbb{Z}_{2} .
$$

Thus if $C$ is round $\beta(C, C)=v(C) \in \mathbb{Z}_{2}$. If $C$ is even

$$
\beta(C, C)=0=v(C)+\frac{1}{2} \chi(C) \in \mathbb{Z}_{2} .
$$

Proof. If $C$ is such that $C_{r}=0$ for $r \neq n$, both sides of the identity are zero.

If the identity holds for finite chain complexes $C, C^{\prime}$ then it also holds for their sum $C \oplus C^{\prime}$, since

$\beta\left(C \oplus C^{\prime}, C \oplus C^{\prime}\right)-\beta(C, C)-\beta\left(C^{\prime}, C^{\prime}\right)=\beta\left(C, C^{\prime}\right)+\beta\left(C^{\prime}, C\right)$

$$
\begin{aligned}
= & \sum_{r} c_{r} c_{r}^{\prime}+\chi_{\text {even }}(C) \chi_{\text {odd }}\left(C^{\prime}\right)+\chi_{\text {odd }}(C) \chi_{\text {even }}\left(C^{\prime}\right) \\
= & \left(v\left(C \oplus C^{\prime}\right)+\frac{1}{2} \chi_{\text {even }}\left(C \oplus C^{\prime}\right)\left(\chi_{\text {even }}\left(C \oplus C^{\prime}\right)-1\right)+\frac{1}{2} \chi_{\text {odd }}\left(C \oplus C^{\prime}\right)\left(\chi_{\text {odd }}\left(C \oplus C^{\prime}\right)-1\right)-\right. \\
& -\left(v(C)+\frac{1}{2} \chi_{\text {even }}(C)\left(\chi_{\text {even }}(C)-1\right)+\frac{1}{2} \chi_{\text {odd }}(C)\left(\chi_{\text {odd }}(C)-1\right)\right)- \\
& -\left(v\left(C^{\prime}\right)+\frac{1}{2} \chi_{\text {even }}\left(C^{\prime}\right)\left(\chi_{\text {even }}\left(C^{\prime}\right)-1\right)+\frac{1}{2} \chi_{\text {odd }}\left(C^{\prime}\right)\left(\chi_{\text {odd }}\left(C^{\prime}\right)-1\right)\right) \in \mathbb{Z}_{2} .
\end{aligned}
$$

Ignoring boundaries $C=C_{0} \oplus S C_{1} \oplus S^{2} C_{2} \oplus \ldots \oplus S^{n} C_{n}$, for some $n \geqslant 0$, so that the identity holds for all finite complexes $C$.

Applying the Lemma we have that for even $C$

$$
\eta(D, C)=\beta(D, D) v(C), \quad \varepsilon(D, C)=v(D) v(C) \in \mathbb{Z}_{2}
$$

and as $D$ is round $\beta(D, D)=v(D)$, so that

$$
\eta(D, C)+\varepsilon(D, C)=0 \in \mathbb{Z}_{2} .
$$

(iv) Expressing $f \otimes g$ as the composite $f \otimes g: C \otimes D \stackrel{f \otimes 1_{D}}{\rightarrow} C^{\prime} \otimes D \stackrel{1_{C^{\prime}} \otimes g}{\longrightarrow} C^{\prime} \otimes D^{\prime}$ 
we have by the logarithmic property of reduced torsion

$$
\tau(f \otimes g)=\tau\left(f \otimes 1_{D}\right)+\tau\left(1_{C^{\prime}} \otimes g\right) \in \tilde{K}_{1}(A \otimes B)
$$

The sign terms may be ignored in the reduced $K_{1}$-group, so that

$$
\tau\left(f \otimes 1_{D}\right)=\tau\left(C\left(f \otimes 1_{D}\right)\right)=\tau(C(f) \otimes D) \in \tilde{K}_{1}(A \otimes B) .
$$

By (ii) above

$$
\tau(C(f) \otimes D)=\tau(C(f)) \otimes[D]+\text { sign term } \in K_{1}(A \otimes B),
$$

so that

$$
\tau\left(f \otimes 1_{D}\right)=\tau(C(f) \otimes D)=\tau(f) \otimes[D] \in \tilde{K}_{1}(A \otimes B) .
$$

Similarly, by (iii)

$$
\tau\left(1_{C^{\prime}} \otimes g\right)=\left[C^{\prime}\right] \otimes \tau(g)=[C] \otimes \tau(g) \in \tilde{K}_{1}(A \otimes B) .
$$

The product formula of Proposition 3.1(i) was first obtained by Gersten [7] (although of course well known prior to that for $\chi$ ), and that of Proposition 3.1 (iv) by Kwun and Szczarba [10]. The topological interpretations are recalled in Proposition 4.5 below.

Proposition 3.1(i) shows that the product $C \otimes D$ of a finitely dominated chain complex $C$ over $A$ and a chain complex $D$ over $B$ which admits a round finite structure is a chain complex over $A \otimes B$ such that

$$
[C \otimes D]=[C] \otimes[D]=[C] \otimes 0=0 \in K_{0}(A \otimes B),
$$

so that $C \otimes D$ also admits a round finite structure. More precisely:

PROPOSITION 3.2.(i) The product of a finitely dominated chain complex $C$ over $A$ and a chain complex $D$ over $B$ with a round finite structure $(G, \theta) \in \mathscr{F}^{r}(D)$ is a chain complex $C \otimes D$ over $A \otimes B$ with a canonical product round finite structure $C \otimes(G, \theta) \in \mathscr{F}^{r}(C \otimes D)$.

(ii) The product $f \otimes g: C \otimes D \rightarrow C^{\prime} \otimes D^{\prime}$ of a chain equivalence $f: C \rightarrow C^{\prime}$ of finitely dominated chain complexes over $A$ and a chain equivalence $g: D \rightarrow D^{\prime}$ of chain complexes over $B$ with round finite structures $(G, \theta) \in \mathscr{F}^{r}(D),\left(G^{\prime}, \theta^{\prime}\right) \in \mathscr{F}^{r}\left(D^{\prime}\right)$ is a chain equivalence of chain complexes over $A \otimes B$ with torsion

$$
\tau(f \otimes g)=[C] \otimes \tau(g) \in K_{1}(A \otimes B)
$$

with respect to the product round finite structures

$$
C \otimes(G, \theta) \in \mathscr{F}^{r}(C \otimes D), \quad C^{\prime} \otimes\left(G^{\prime}, \theta^{\prime}\right) \in \mathscr{F}^{r}\left(C^{\prime} \otimes D^{\prime}\right),
$$

where $[C]=\left[C^{\prime}\right] \in K_{0}(A)$ and $\tau(\mathrm{g}) \in K_{1}(B)$.

Proof. This occupies the rest of the Section. In (i) we shall define the product round finite structure $C \otimes(D, 1) \in \mathscr{F}^{r}(C \otimes D)$ for a round finite chain complex $D$ over $B$. 
Then in (ii) we shall prove the torsion product formula

$$
\tau\left(f \otimes g: C \otimes D \rightarrow C^{\prime} \otimes D^{\prime}\right)=[C] \otimes \tau\left(g: D \rightarrow D^{\prime}\right) \in K_{1}(A \otimes B)
$$

for any chain equivalence $g: D \rightarrow D^{\prime}$ of round finite chain complexes, with respect to the round finite structures $C \otimes(D, 1) \in \mathscr{F}^{r}(C \otimes D), C^{\prime} \otimes\left(D^{\prime}, 1\right) \in \mathscr{F}^{r}\left(C^{\prime} \otimes D^{\prime}\right)$.

For any chain complex $D$ over $B$ with a round finite structure $(G, \theta) \in \mathscr{F}^{r}(D)$ the product round finite structure $C \otimes(G, \theta) \in \mathscr{F}^{r}(C \otimes D)$ can then be defined using $C \otimes(G, 1)=(F, \phi) \in \mathscr{F}^{r}(C \otimes G)$ to be

$$
C \otimes(G, \theta)=((1 \otimes \theta) \phi: F \rightarrow C \otimes G \rightarrow C \otimes D) \in \mathscr{F}^{r}(C \otimes D) .
$$

(i) It suffices to consider only the case of a rounded finite chain complex $D$ over $B$, since by Proposition 1.7(ii) for any round finite chain complex $D$ over $B$ there exists a contractible finite chain complex $D^{\prime}$ such that $D \otimes D^{\prime}$ is rounded and

$$
\tau\left((10): D \oplus D^{\prime} \rightarrow D\right)=0 \in K_{1}(B) .
$$

If $C \otimes\left(D \oplus D^{\prime}, 1\right)=(F, \phi) \in \mathscr{F}^{*}\left(C \otimes\left(D \oplus D^{\prime}\right)\right)$ is already defined let $C \otimes(D, 1)=$ $\left(F,(1 \otimes(1,0)) \phi: F \rightarrow C \otimes\left(D \oplus D^{\prime}\right) \rightarrow C \otimes D\right) \in \mathscr{F}^{r}(C \otimes D)$.

Let then $D$ be a rounded finite chain complex over B. By Proposition 1.7(i) there exists a contractible finite chain complex $D_{\Delta}$ over $B$ with the same chain modules $\left(D_{r} \mid r \geqslant 0\right\}$, and the differentials $\left\{d_{\Delta} \in \operatorname{Hom}_{B}\left(D_{r}, D_{r-1}\right) \mid r \geqslant 1\right\}$ can be chosen such that

$$
\tau\left(D_{\Delta}\right)=0 \in K_{1}(B)
$$

In dealing with the finitely dominated chain complex $C$ over $A$ it is convenient to work with the idempotent completion $\underline{P}(A)$ of the additive category $\mathscr{A}=F(A)$ of based f.g. free $A$-modules. An object in $\underline{\underline{P}}(A)$ is a pair $(E, p)$ consisting of a based f.g. free $A$-module $E$ and an $A$-module morphism $p \in \operatorname{Hom}_{A}(E, E)$ which is a projection

$$
p^{2}=p: E \rightarrow E
$$

A morphism in $\underline{\underline{P}}(A)$

$$
f:(E, p) \rightarrow\left(E^{\prime}, p^{\prime}\right)
$$

is an $A$-module morphism $f \in \operatorname{Hom}_{A}\left(E, E^{\prime}\right)$ such that

$$
p^{\prime} f p=f: E \rightarrow E^{\prime} .
$$

The additive functor

$P(A) \rightarrow\{$ f.g. projective $A$-modules $\} ;(E, p) \rightarrow \operatorname{im}(p: E \rightarrow E)$

is an equivalence of additive categories.

A finite idempotent chain complex over $A(E, p)$ is a finite chain complex in $\underline{\underline{P}}(A)$

$$
(E, p): \ldots \rightarrow 0 \rightarrow\left(E_{n}, p_{n}\right) \stackrel{d}{-}\left(E_{n-1}, p_{n-1}\right) \rightarrow \ldots \rightarrow\left(E_{0}, p_{0}\right) .
$$

The chain homotopy theory of finite idempotent chain complexes is defined in the 
obvious way, with a bijection of sets of chain equivalence classes

$\{$ finite idempotent chain complexes over $A$ \}

$\rightarrow\{$ finitely dominated chain complexes over $A\}$;

$$
(E, p) \rightarrow \operatorname{im}(p: E \rightarrow E)
$$

See Ranicki [15] for a detailed exposition.

Given a finite idempotent chain complex $(E, p)$ over $A$ and a rounded finite chain complex $D$ over $B$ define a round finite chain complex over $A \otimes B$

$$
F=(E, p) \otimes D
$$

by

$$
\begin{aligned}
& d_{F}: F_{r}=(E \otimes D)_{r}=\Sigma_{s=0}^{r} E_{s} \otimes D_{r-s} \rightarrow F_{r-1} ; \\
& x \otimes y \rightarrow p(x) \otimes d_{D}(y)+(1-p)(x) \otimes d_{\Delta}(y)+(-)^{r-s} d_{E}(x) \otimes y,
\end{aligned}
$$

with $\left\{d_{D} \in \operatorname{Hom}_{B}\left(D_{r}, D_{r-1}\right)^{\pi} r \geqslant 1\right\}$ the differentials of $D$ and $\left\{d_{A} \in \operatorname{Hom}_{B}\left(D_{r}, D_{r-1}\right)\right.$ $\mid r \geqslant 1\}$ the differentials of $D_{\Delta}$ (as above). For example, if $p=1: E \rightarrow E$ then $F=F \otimes D$. As an unbased chain complex over $B$

$$
F=\operatorname{im}(p) \otimes D \oplus \operatorname{im}(1-p) \otimes D_{\Delta},
$$

and the projection

$$
F \rightarrow \operatorname{im}(p) \otimes D ; \quad x \otimes y \rightarrow p(x) \otimes y
$$

is a chain equivalence (since it has contractible kernel $\operatorname{im}(1-p) \otimes D_{\Delta}$ ).

A finite idempotent chain complex $(E, p)$ over $A$ is even if

$$
\operatorname{rank}_{A}\left(E_{r}\right) \equiv 0(\bmod 2)(r \geqslant 0) \text {. }
$$

For any finitely dominated chain complex $C$ over $A$ there exists a triple $(E, p, \theta)$ consisting of an even idempotent finite chain complex $(E, p)$ over $A$ and a chain equivalence $\theta: \operatorname{im}(p) \rightarrow C$. (Choose a bounded f.g. projective chain complex $P$ over $A$ chain equivalent to $C$, and let $\left\{Q_{r} \mid r \geqslant 0\right\}$ be a sequence of f.g. projective A-modules such that $P_{r} \oplus Q_{r}$ is a f.g. free $A$-module of even rank if $P_{r}$ is non-zero and $Q_{r}=0$ if $P_{r}=0$. Then $E=P \oplus Q$ as an unbased chain complex, with

$$
\begin{aligned}
& d_{E}=\left(\begin{array}{ll}
d_{P} & 0 \\
0 & 0
\end{array}\right): E_{r}=P_{r} \oplus Q_{r} \rightarrow E_{r-1}=P_{r-1} \oplus Q_{r-1}, \\
& \left.p=\left(\begin{array}{ll}
1 & 0 \\
0 & 0
\end{array}\right): E_{r}=P_{r} \oplus Q_{r} \rightarrow E_{r}=P_{r} \oplus Q_{r}, \operatorname{im}(p: E \rightarrow E)=P\right) .
\end{aligned}
$$

The product round finite structure $C \otimes(D, 1)=(F, \phi) \in \mathscr{F}^{\mathrm{r}}(C \otimes D)$ is defined 
using any such triple $(E, p, \theta)$ by

$$
\phi: F=(E, p) \otimes D \stackrel{\text { projection }}{\longrightarrow} \operatorname{im}(p) \otimes D \stackrel{\theta \otimes 1}{\longrightarrow} C \otimes D .
$$

We have to show that $(F, \phi) \in \mathscr{F}^{r}(C \otimes D)$ is independent of the choice of $(E, p, \theta)$. If $(E, p, \theta),\left(E^{\prime}, p^{\prime}, \theta^{\prime}\right)$ are two such choices the chain equivalence of even idempotent finite complexes

$$
f=\phi^{\prime-1} \phi \oplus 0:(E, p) \rightarrow\left(E^{\prime}, p^{\prime}\right)
$$

is such that

$$
\begin{gathered}
(F, \phi)-\left(F^{\prime}, \phi^{\prime}\right)=\tau\left(f \otimes 1: F=(E, p) \otimes D \rightarrow F^{\prime}=\left(E^{\prime}, p^{\prime}\right) \otimes D\right) \\
\in K_{1}(A \otimes B) .
\end{gathered}
$$

We thus have to show that $\tau(f \otimes 1)=0 \in K_{1}(A \otimes B)$. We consider first the special case of contractible $C$ :

LEMMA If $(E, p)$ is an even finite idempotent chain complex over $A$ such that $P=\operatorname{im}(p: E \rightarrow E)$ is a contractible chain complex over $A$ then $F=(E, p) \otimes D$ is a contractible finite chain complex over $A \otimes B$ with torsion $\tau(F)=0 \in K_{1}(A \otimes B)$.

Proof. Choose a chain contraction $\Gamma: 0 \simeq 1: P \rightarrow P$ and define an isomorphism of contractible finite chain complexes over $A \otimes B$

$$
h: F \rightarrow E \otimes D_{\Lambda}
$$

by the $A \otimes B$-module automorphisms

$$
\begin{aligned}
& h_{r}: F_{r}=\Sigma_{s=0}^{r} E_{s} \otimes D_{r-s} \rightarrow\left(E \otimes D_{\Delta}\right)_{r}=\Sigma_{s=0}^{r} E_{s} \otimes D_{r-s} \\
& \left.x \otimes y \rightarrow x \otimes y+(-)^{r-s} \Gamma p(x) \otimes\left(d_{\Delta}-d_{D}\right)(y)\right)(r \geqslant 0)
\end{aligned}
$$

so that

$$
\tau(F)=\tau\left(E \otimes D_{\Delta}\right)-\sum_{r=0}^{\infty}(-)^{r} \tau\left(h_{r}:(E \otimes D)_{r} \rightarrow(E \otimes D)_{r}\right) \in K_{1}(A \otimes B) .
$$

As $E$ is even

$$
\begin{aligned}
\tau\left(E \otimes D_{\Delta}\right) & =[E] \otimes \tau\left(D_{\Delta}\right) \text { (by Proposition 3.1(ii)) } \\
& =[E] \otimes 0=0 \in K_{1}(A \otimes B) .
\end{aligned}
$$

The f.g. projective $A \otimes B$-modules $M_{r}, N_{r}(r \geqslant 0)$ defined by

$$
\begin{aligned}
& M_{r}=\sum_{s=0}^{r} \operatorname{ker}\left(d_{P}: P_{s} \rightarrow P_{s-1}\right) \otimes D_{r-s}, \\
& N_{r}=\sum_{s=0}^{r}\left(\operatorname{ker}\left(\Gamma: P_{s} \rightarrow P_{s+1}\right) \oplus \operatorname{im}\left(1-p: E_{s} \rightarrow E_{s}\right)\right) \otimes D_{r-s}
\end{aligned}
$$

are such that

$$
\begin{aligned}
h_{r}= & \left(\begin{array}{cc}
1 & 0 \\
\sum_{s=0}^{r}(-)^{r-s} \Gamma \otimes\left(d_{\Delta}-d_{D}\right) & 1
\end{array}\right) \\
& :(E \otimes D)_{r}=M_{r} \oplus N_{r} \rightarrow M_{r} \oplus N_{r} \quad(r \geqslant 0) .
\end{aligned}
$$


Thus $\tau\left(h_{r}\right)=0 \in K_{1}(A \otimes B)$, and $\tau(F)=0 \in K_{1}(A \otimes B)$.

The algebraic mapping cone of a chain map of even idempotent finite chain complexes over $A$

$$
f:(E, p) \rightarrow\left(E^{\prime}, p^{\prime}\right)
$$

is an even idempotent finite complex $(C(f), q)$ with

$$
q=\left(\begin{array}{ll}
p^{\prime} & 0 \\
0 & p
\end{array}\right): C(f)_{r}=E_{r}^{\prime} \oplus E_{r-1} \rightarrow E_{r}^{\prime} \oplus E_{r-1} \quad(r \geqslant 0) .
$$

The rearrangement isomorphism

$$
(C(f), q) \otimes D \rightarrow C\left(f \otimes 1:(E, p) \otimes D \rightarrow\left(E^{\prime}, p^{\prime}\right) \otimes D\right)
$$

has torsion $\mu\left(E^{\prime}, S E, D\right) \tau(-1: A \otimes B \rightarrow A \otimes B) \in K_{1}(A \otimes B)$, which is 0 since $E$ and $E^{\prime}$ are even. If $f$ is a chain equivalence (i.e. if $f \mid: \operatorname{im}\left(p^{\prime}\right)$ is a chain equivalence) then $\operatorname{im}(q)$ is contractible and

$$
f \otimes 1: F=(E, p) \otimes D \rightarrow F^{\prime}=\left(E^{\prime}, p^{\prime}\right) \otimes D
$$

is a chain equivalence of even round finite chain complexes over $A \otimes B$ with torsion

$$
\begin{aligned}
\tau(f \otimes 1) & =\tau(C(f \otimes 1)) \\
& =\tau((C(f), q) \otimes D) \\
& =0 \in K_{1}(A \otimes B), \text { by the Lemma, }
\end{aligned}
$$

It follows that $(F, \phi)=\left(F^{\prime}, \phi^{\prime}\right) \in \mathscr{F}^{r}(C \otimes D)$, so that the round infinite structure defined on $C \otimes D$ is indeed canonical.

(ii) As for (i) it suffices to consider the special case when $D$ and $D^{\prime}$ are rounded finite chain complexes over $B$. By the logarithmic property of torsion

$$
\begin{aligned}
\tau\left(f \otimes g: C \otimes D \rightarrow C^{\prime} \otimes D^{\prime}\right)= & \tau\left(f \otimes g: C \otimes D \stackrel{1 \otimes g}{\longrightarrow} C \otimes D^{\prime} \stackrel{f \otimes 1}{\longrightarrow} C^{\prime} \otimes D\right) \\
= & \tau\left(f \otimes 1: C \otimes D^{\prime} \rightarrow C^{\prime} \otimes D^{\prime}\right)+ \\
& +\tau\left(1 \otimes g: C \otimes D \rightarrow C \otimes D^{\prime}\right) \in K_{1}(A \otimes B) .
\end{aligned}
$$

Let $(F, \phi) \in \mathscr{F}^{r}(C \otimes D),\left(F^{\prime}, \phi^{\prime}\right) \in \mathscr{F}^{r}\left(C^{\prime} \otimes D\right)$ be the product round finite structures. By definition

$$
\tau(f \otimes 1)=\tau\left(F \stackrel{\phi}{\longrightarrow} C \otimes D \stackrel{f \otimes 1}{\longrightarrow} C^{\prime} \otimes D \stackrel{\phi^{\prime-1}}{\longrightarrow} F^{\prime}\right) \in K_{1}(A \otimes B) .
$$

The proof in (i) above that $(F=(E, p) \otimes D, \phi) \in \mathscr{F}^{r}(C \otimes D)$ is independent of the choice of $(E, p)$ includes a proof that $\tau(f \otimes 1)=0 \in K_{1}(A \otimes B)$.

We shall prove that $\tau(1 \otimes g)=[C] \otimes \tau(g) \in K_{1}(A \otimes B)$ using the following generalization of the product formula of Proposition 3.1(iii), which is the special case $D=0$. 
LEMMA. The product $F=(E, p) \otimes D$ of an even finite idempotent chain romplex $(E, p)$ over $A$ and a contractible finite chain complex $D$ over $B$ is a contractible finite chain complex over $A \otimes B$ with torsion

$$
\tau(F)=[\operatorname{im}(p)] \otimes \tau(D) \in K_{1}(A \otimes B) .
$$

Proof. Choose chain contractions

$$
\Gamma_{D}: 0 \simeq 1: D \rightarrow D, \quad \Gamma_{\Delta}: 0 \simeq 1: D_{\Delta} \rightarrow D_{\Delta},
$$

and use them to define a chain contraction

$$
\Gamma_{F}=p \otimes \Gamma_{D}+(1-p) \otimes \Gamma_{\Delta}: 0 \simeq 1: F \rightarrow F .
$$

If $E$ is 0 -dimensional the rearrangement isomorphisms are such that

$$
\tau\left(\left((E \otimes D)_{\text {even }} \rightarrow E_{0} \otimes D_{\text {even }}\right)=0, \tau\left((E \otimes D)_{\text {odd }} \rightarrow E_{0} \otimes D_{\text {odd }}\right)=0 \in K_{1}(A \otimes B),\right.
$$

so that

$$
\begin{aligned}
\tau(F)= & \tau\left(d_{F}+\Gamma_{F}: F_{\text {odd }}=(E \otimes D)_{\text {odd }} \rightarrow F_{\text {even }}=(E \otimes D)_{\text {even }}\right) \\
= & \tau\left(p \otimes\left(d_{D}+\Gamma_{D}\right)+(1-p) \otimes\left(d_{\Delta}+\Gamma_{\Delta}\right): E_{0} \otimes D_{\text {odd }} \rightarrow E_{0} \otimes D_{\text {even }}\right) \\
= & \tau\left(p \otimes\left(d_{D}+\Gamma_{D}\right)\left(d_{\Delta}+\Gamma_{\Delta}\right)^{-1}+(1-p) \otimes 1: E_{0} \otimes D_{\text {even }} \rightarrow \mathrm{E}_{0} \otimes D_{\text {even }}\right) \\
& \quad\left(\text { since } \tau\left(d_{\Delta}+\Gamma_{\Delta}: D_{\text {odd }} \rightarrow D_{\text {even }}\right)=\tau\left(D_{\Delta}\right)=0 \in K_{1}(B)\right) \\
= & {[i m(p)] \otimes \tau\left(\left(d_{D}+\Gamma_{\mathrm{D}}\right)\left(d_{\Delta}+\Gamma_{\Delta}\right)^{-1}: D_{\text {even }} \rightarrow D_{\text {even }}\right) } \\
= & {[i m(p)] \otimes \tau\left(d_{D}+\Gamma_{D}: D_{\text {odd }} \rightarrow D_{\text {even }}\right) } \\
= & {[i m(p)] \otimes \tau(D) \in K_{1}(A \otimes B) . }
\end{aligned}
$$

Assume inductively that $\tau(F)=[\operatorname{im}(p: E \rightarrow E)] \otimes \tau(D) \in K_{1}(A \otimes B)$ if $E$ is of dimension $<n$, and that the dimension of $E$ is $n$. Let $E^{\prime}$ be the $(n-1)$-skelton of $E$, so that there is defined a short exact sequence of finite idempotent chain complexes over $A$

$$
0 \rightarrow\left(E^{\prime}, p^{\prime}\right) \stackrel{i}{\longrightarrow}(E, p) \stackrel{j}{\longrightarrow}\left(S^{n} E_{n}, p_{n}\right) \rightarrow 0
$$

Applying $-\otimes D$ there is obtained a short exact sequence of finite chain complexes over $A \otimes B$

$$
0 \rightarrow\left(E^{\prime}, p^{\prime}\right) \otimes D \stackrel{i \otimes 1}{\longrightarrow}(E, p) \otimes D \stackrel{j \otimes 1}{\longrightarrow}\left(S^{n} E_{n}, p_{n}\right) \otimes D \rightarrow 0 .
$$

By the torsion sum formula of Proposition 2.3 of Part I and the inductive hypothesis

$$
\begin{aligned}
\tau((E, p) \otimes D)= & \tau\left(\left(E^{\prime}, p^{\prime}\right) \otimes D\right)+\tau\left(\left(S^{n} E_{n}, p_{n}\right) \otimes D\right) \\
& \quad(\text { the sign term vanishes since } E \text { is even }) \\
= & {\left[\operatorname{im}\left(p^{\prime}\right)\right] \otimes \tau(D)+(-)^{n}\left[\operatorname{im}\left(p_{n}\right)\right] \otimes \tau(D) } \\
= & {[\operatorname{im}(p)] \otimes \tau(D) \in K_{1}(A \otimes B) . }
\end{aligned}
$$


The algebraic mapping cone of a chain equivalence $g: D \rightarrow D^{\prime}$ of round finite chain complexes over $B$ is a contractible finite chain complex $C(g)$ over $B$, so that

$$
\tau((E, p) \otimes C(g))=[\operatorname{im}(p)] \otimes \tau(C(g)) \in K_{1}(A \otimes B)
$$

by the Lemma. The round finite complexes $(E, p) \otimes D,(E, p) \otimes D^{\prime}$ over $A \otimes B$ are constructed using any contractible chain complexes $D_{\Delta}, D_{\Delta}^{\prime}$ over $\boldsymbol{B}$ with the chain modules of $D, D^{\prime}$ respectively, and such that

$$
\tau\left(D_{\Delta}\right)=\tau\left(D_{\Delta}^{\prime}\right)=0 \in K_{1}(B) .
$$

Now $C(g)$ has the chain modules of $D^{\prime} \oplus S D$, but

$$
\tau\left(D_{\Delta}^{\prime} \oplus S D_{\Delta}\right)=\beta\left(D^{\prime}, S D\right) \tau(-1: B \rightarrow B) \in K_{1}(B)
$$

so that $C(g)_{\Delta}$ cannot in general be chosen to be $D_{\Delta}^{\prime} \oplus S D_{\Delta}$. We shall construct $(E, p) \otimes C(g)$ using the acyclic finite complex

$$
C(g)_{\Delta}=D_{\Delta}^{\prime}, \oplus D_{\Delta}
$$

with $D_{\Delta}^{\prime}$, defined as follows. Choose an automorphism $\alpha \in \operatorname{Hom}_{B}\left(D_{n}^{\prime}, D_{n}^{\prime}\right)$ of a chain module $D_{n}^{\prime}$ of $D^{\prime}$ such that

$$
\tau(\alpha)=\beta\left(D^{\prime}, S D\right) \tau(-1: B \rightarrow B) \in K_{1}(B) .
$$

Define $D_{\Delta}^{\prime}$, by

$$
d_{\Delta^{\prime}}=\left\{\begin{array} { l } 
{ d _ { \Delta } } \\
{ d _ { \Delta } \alpha ^ { - 1 } : D _ { r } ^ { \prime } \rightarrow D _ { r - 1 } ^ { \prime } } \\
{ \alpha d _ { \Delta } }
\end{array} \text { if } \left\{\begin{array}{l}
\mathrm{r} \neq n, n+1 \\
r=n \\
\mathrm{r}=n+1,
\end{array}\right.\right.
$$

so that there is defined an isomorphism of contractible finite chain complexes over $B$

$$
h: D_{\Delta}^{\prime} \stackrel{\sim}{\longrightarrow} D_{\Delta}^{\prime}
$$

with

$$
h=\left\{\begin{array} { l } 
{ 1 } \\
{ \alpha }
\end{array} : D _ { r } ^ { \prime } \rightarrow D _ { r } ^ { \prime } \quad \text { if } \left\{\begin{array}{l}
r \neq n \\
r=n
\end{array}\right.\right.
$$

The torsion of $h$ is given by

$$
\tau(h)=\tau\left(D_{\Delta^{\prime}}^{\prime}\right)=(-)^{n} \tau(\alpha)=\beta\left(D^{\prime}, S D\right) \tau(-1: B \rightarrow B) \in K_{1}(B)
$$

and

$$
\begin{aligned}
\tau\left(C(g)_{\Delta}\right) & =\tau\left(D_{\Delta^{\prime}}^{\prime}\right)+\tau\left(S D_{\Delta}\right)+\beta\left(D^{\prime}, S D\right) \tau(-1: B \rightarrow B) \\
& =0 \in K_{1}(B) .
\end{aligned}
$$

The isomorphism of contractible finite chain complexes over $A \otimes B$

$$
\begin{aligned}
& k:(E, p) \otimes C(g) \rightarrow C\left(1 \otimes g:(E, p) \otimes D \rightarrow(E, p) \otimes D^{\prime}\right) \\
& x \otimes\left(y^{\prime}, y\right) \rightarrow p(x) \otimes\left(y^{\prime}, y\right)+(1-p)(x) \otimes\left(h\left(y^{\prime}\right), y\right)
\end{aligned}
$$


has torsion

$$
\begin{aligned}
\tau(k) & =\tau(C(1 \otimes g))-\tau((E, p) \otimes C(g)) \\
& \left.=\Sigma_{r=0}^{\infty}(-)^{r} \tau\left(k_{r}:(E, p) \otimes C(g)\right)_{r} \rightarrow C(1 \otimes g)_{r}\right)
\end{aligned}
$$

(by Proposition 1.2(iii))

$$
\begin{aligned}
= & \sum_{r=n}^{\infty}(-)^{r} \tau\left(k_{r}: E_{r-n} \otimes D_{n}^{\prime} \rightarrow E_{r-n} \otimes D_{n}^{\prime} ;\right. \\
& \left.\quad x \otimes y^{\prime} \rightarrow p(x) \otimes y^{\prime}+(1-p)(x) \otimes h\left(y^{\prime}\right)\right) \\
= & \sum_{r=n}^{\infty}(-)^{r}\left[\operatorname{im}\left(1-p: E_{r-n} \rightarrow E_{r-n}\right)\right] \otimes \tau\left(\alpha: D_{n}^{\prime} \rightarrow D_{n}^{\prime}\right) \\
= & {[\operatorname{im}(p)] \otimes \beta\left(D^{\prime}, S D\right) \tau(-1: B \rightarrow B) \in K_{1}(A \otimes B) . }
\end{aligned}
$$

Thus

$$
\begin{aligned}
\tau\left(1 \otimes g: C \otimes D \rightarrow C \otimes D^{\prime}\right) & =\tau\left(1 \otimes g:(E, p) \otimes D \rightarrow(E, p) \otimes D^{\prime}\right) \\
& =\tau(C(1 \otimes g)) \\
& =\tau((E, p) \otimes C(g))+[\operatorname{im}(p)] \otimes\left(\beta\left(D^{\prime}, S D\right) \tau(-1: B \rightarrow B)\right) \\
& =[\operatorname{im}(p)] \otimes\left(\tau(C(g))+\beta\left(D^{\prime}, S D\right) \tau(-1: B \rightarrow B)\right) \\
& =[C] \otimes \tau(g) \in K_{1}(\ddot{A} \otimes B) .
\end{aligned}
$$

In the special case when $f: C \rightarrow C^{\prime}=C, g: D \rightarrow D^{\prime}=D$ the product formula of Proposition 3.2(ii) agrees with the product formula obtained by Gersten [8] (cf. Proposition 5.2 below).

\section{Torsion for CW Complexes}

Let $\hat{X}$ be a regular cover of a $C W$ complex $X$ with group of covering translations $\pi$. The cellular chain complex of $\tilde{X}$ is the free chain complex over $\mathbb{Z}[\pi]$

$$
C(\tilde{X}): \cdots \rightarrow C_{r+1}(\tilde{X}) \stackrel{d}{\longrightarrow} C_{r}(\bar{X}) \stackrel{d}{\longrightarrow} C_{r-1}(\bar{X}) \rightarrow \cdots \rightarrow C_{0}(\tilde{X})
$$

defined in the usual manner, with

$$
C_{r}(\tilde{X})=H_{r}\left(\tilde{X}^{(r)}, \tilde{X}^{(r-1)}\right) \quad(r \geqslant 0)
$$

a free $\mathbb{Z}[\pi]$-module with one generator for each $r$-cell of $X$.

We shall be mainly concerned with connected $\mathrm{CW}$ complexes $X$, with $\tilde{X}$ the universal cover and $\pi=\pi_{1}(X)$ the fundamental group. A geometric base for $X$ is a base for the free $\mathbb{Z}[\pi]$-module $\sum_{r=0}^{\infty} C_{r}(\tilde{X})$ such that each base element is the Hurewicz image $\tilde{\phi}_{*}\left[e^{r}\right] \in C_{r}(\tilde{X})$ of a fundamental class $\left[e^{r}\right]=$ $\pm 1 \in H_{r}\left(e^{r}, \partial e^{r}\right)=\mathbb{Z}$ under a lift $\tilde{\phi}:\left(e^{r}, \partial e^{r}\right) \rightarrow\left(\bar{X}^{(r)}, \bar{X}^{(r-1)}\right)$ of a characteristic map $\phi:\left(e^{r}, \partial e^{r}\right) \rightarrow\left(X^{(r)}, X^{(r-1)}\right)$. Geometric base elements are unique up to mulitplication by $\pm g(g \in \pi)$. A geometric base for a finite $\mathrm{CW}$ complex $X$ determines a finite chain complex $C(\tilde{X})$ over $\mathbb{Z}[\pi]$.

A map of (connected) CW complexes $f: X \rightarrow Y$ induces a morphism of fundamental groups

$$
f_{*}=\alpha: \pi_{1}(X)=\pi \rightarrow \pi_{1}(Y)=\rho
$$


which is unique up to composition with inner automorphisms if base points are ignored. The universal cover $\bar{Y}$ of $Y$ pulls back to a cover $f^{*} \hat{Y}$ of $X$ such that $f$ lifts to a $\rho$-equivariant map $\tilde{f}: f^{*} \tilde{Y} \rightarrow \tilde{X}$ inducing a chain map over $\mathbb{Z}[\rho]$

$$
\tilde{f}: C\left(f^{*} \tilde{Y}\right)=\alpha_{1} C(\tilde{X}) \rightarrow C(\tilde{Y}) .
$$

The map $f: X \rightarrow Y$ is a homotopy equivalence if and only if $\alpha: \pi \rightarrow \rho$ is an isomorphism and $\tilde{f}: \alpha, C(\tilde{X}) \rightarrow C(\tilde{Y})$ is a chain equivalence.

A finite domination $(Y, f, g, h)$ of a $\mathrm{CW}$ complex $X$ consists of a finite $\mathrm{CW}$ complex $Y$, maps

$$
f: X \rightarrow Y, \quad g: Y \rightarrow X
$$

and a homotopy

$$
h: g f \simeq 1: X \rightarrow X .
$$

A CW complex $X$ is finitely dominated if it admits a finite domination.

Let $X$ be a connected CW complex with universal cover $\tilde{X}$ and fundamental group $\pi_{1}(X)=\pi$. A finite domination $(Y, f, g, h)$ of $X$ and a choice of geometric base for $Y$ determine a finite domination of the chain complex $C(\tilde{X})$ over $\mathbb{Z}[\pi]$

$$
(C(\tilde{Y}), \tilde{f}: C(\tilde{X}) \rightarrow C(\tilde{Y}), g: C(\tilde{Y}) \rightarrow C(\tilde{X}), \tilde{h}: \tilde{g} \tilde{f} \simeq 1: C(\tilde{X}) \rightarrow C(\tilde{X})),
$$

where $\tilde{Y}=g^{*} \tilde{X}$ is the pullback cover of $Y$. The projective class of a finitely dominated $\mathrm{CW}$ complex $X$ is defined by

$$
[X]=[C(\tilde{X})] \in K_{0}(\mathbb{Z}[\pi]) .
$$

This is a homotopy invariant which can be expressed as

$$
[X]=(\chi(X),[X]) \in K_{0}(\mathbb{Z}[\pi])=K_{0}(\mathbb{Z}) \oplus \tilde{K}_{0}(\mathbb{Z}[\pi]),
$$

with $\chi(X)=\chi(C(X)) \in K_{0}(\mathbb{Z})=\mathbb{Z}$ the Euler characteristic of $X$ and $[X] \in \widetilde{K}_{0}(\mathbb{Z}[\pi]$ the reduced projective class.

PROPOSITION 4.1 (Wall [21]). (i) $A C W$ complex $X$ is finitely dominated if and only if $\pi_{1}(X)=\pi$ is finitely presented and $C(\tilde{X})$ is finitely dominated.

(ii) A finitely dominated $C W$ complex $X$ is homotopy equivalent to a finite $C W$ complex if and only if $[X]=0 \in \tilde{K}_{0}(\mathbb{Z}[\pi])$, i.e., if and only if $C(\bar{X})$ is chain equivalent to a finite complex. The reduced projective class $[X] \in \bar{K}_{0}(\mathbb{Z}[\pi])$ is the finiteness obstruction of $X$.

The Whitehead group of a group $\pi$ is defined as usual by

$$
\mathrm{Wh}(\pi)=K_{1}(\mathbb{Z}[\pi]) /\{ \pm \pi\}
$$

If $X$ is a connected finite CW complex with $\pi_{1}(X)=\pi$ and $C, C^{\prime}$ are the finite chain complexes over $\mathbb{Z}[\pi]$ defined by the cellular chain complex $C(\bar{X})$ of the universal cover $\tilde{X}$ and two different choices of geometric base then

$$
\tau\left(1: C \rightarrow C^{\prime}\right) \in\{ \pm \pi\} \subseteq K_{1}(\mathbb{Z}[\pi]),
$$

and so has image $0 \in \mathrm{Wh}(\pi)$. 
The (Whitehead) torsion of a homotopy equivalence $f: X \rightarrow Y$ of finite $\mathrm{CW}$ complexes is defined as usual by

$$
\tau(f)=\tau(\tilde{f}: C(\tilde{X}) \rightarrow C(\tilde{Y})) \in \mathrm{Wh}(\pi)
$$

with $\tilde{f}: \tilde{X} \rightarrow \tilde{Y}$ any lift of $f$ to a $\pi$-equivariant map of the universal covers, identifying $\pi=\pi_{1}(X)$ with $\pi_{1}(Y)$ via the isomorphism $f_{*}: \pi_{1}(X) \rightarrow \pi_{1}(Y)$, with any geometric bases for $C(\bar{X})$ and $C(\bar{Y})$. The element $\tau(f) \in W h(\pi)$ is independent of the choices made in its definition.

A finite structure on a CW complex $X$ is an equivalence class of pairs

(finite $\mathrm{CW}$ complex $F$, homotopy equivalence $f: F \rightarrow X$ )

under the equivalence relation

$$
(F, f) \sim\left(F^{\prime}, f^{\prime}\right) \text {, if } \tau\left(f^{\prime-1} f: F \rightarrow F^{\prime}\right)=0 \in \mathrm{Wh}(\pi) \quad\left(\pi=\pi_{1}(X)\right) .
$$

The finite structure set $\mathscr{F}(X)$ of a CW complex $X$ is the set (possibly empty) of finite structures on $X$.

PROPOSITION 4.2. (i) $\mathscr{F}(X)$ is nonempty if and only if $X$ is finitely dominated and $[X]=0 \in \hat{K}_{0}(\mathbb{Z}[\pi])$.

(ii) If $\mathscr{F}(X)$ is nonempty there is defined a transitive Wh( $\pi)$-action $\mathrm{Wh}(\pi) \times \mathscr{F}(X) \rightarrow$ $\mathscr{F}(X)$;

$$
(\tau(g: G \rightarrow F),(F, f)) \rightarrow(G, f g: G \rightarrow X) .
$$

A choice of base point $\left(F_{0}, f_{0}\right)$ determines an abelian group structure of $\mathscr{F}(X)$ with an isomorphism

$$
\mathscr{F}(X) \rightarrow \text { Wh }(\pi) ;(F, f) \rightarrow \tau\left(f^{-1} f_{0}: F_{0} \rightarrow F\right) .
$$

A (Whitehead) finite structure on a $\mathbb{Z}[\pi]$-module chain complex $C$ is an equivalence class of pairs

(finite $\mathbb{Z}\{\pi\}$-module chain complex $F$, chain equivalence $\phi: F \rightarrow C$ ) under the equivalence relation

$$
(F, \phi) \sim\left(F^{\prime}, \phi^{\prime}\right) \quad \text { if } \tau\left(\phi^{\prime-1} \phi: F \rightarrow F^{\prime}\right)=0 \in \mathrm{Wh}(\pi) .
$$

The (Whitehead) finite structure set $\mathscr{F}^{\mathrm{Wh}}(C)$ of a $\mathbb{Z}[\pi]$-module chain complex $C$ is the set (possibly empty) of Whitehead finite structures on $C$. The evident analogue of Proposition 1.6 holds with $\mathrm{Wh}(\pi)$ and $\mathscr{F}^{\mathrm{wh}}(C)$ in place of $K_{1}(A)$ and $\mathscr{F}(C)$.

PROPOSITION 4.3. The finite structure set $\mathscr{F}(X)$ of a $C W$ complex $X$ is in natural bijective correspondence with the finite structure set $\mathscr{F}^{\mathrm{wh}}(C(\bar{X}))$ of the cellular $\mathbb{Z}[\pi]$ module chain complex $C(\hat{X})$ of the universal cover $\bar{X}$, with $\pi=\pi_{1}(X)$. If the sets are nonempty there is defined a natural isomorphism of affine $\mathrm{Wh}(\pi)$-sets

$$
\mathscr{F}(X) \rightarrow \mathscr{F}^{\mathrm{wh}}(C(\tilde{X})) ; \quad(F, f: F \rightarrow X) \rightarrow(C(\bar{F}), \tilde{f}: C(\tilde{F}) \rightarrow C(\bar{X})) .
$$

A finite CW complex $X$ is round if

$$
\chi(X)=0 \in \mathbb{Z}
$$


and there is given a choice of geometric base for $C(\bar{X})$, so that $C(\tilde{X})$ is a round finite $\mathbb{Z}[\pi]$-module chain complex. As usual, $\bar{X}$ is the universal cover of $X$ and $\pi=\pi_{1}(X)$ is the fundamental group.

The torsion of a homotopy equivalence $f: X \rightarrow Y$ of round finite $\mathrm{CW}$ complexes (meaning a homotopy equivalence of the underlying finite $\mathrm{CW}$ complexes) is defined by

$$
\tau(f)=\tau(\tilde{f}: C(\tilde{X}) \rightarrow C(\tilde{Y})) \in K_{1}(\mathbb{Z}[\pi])
$$

using any lift of $f$ to a $\pi$-equivariant map $\tilde{f}: \tilde{X} \rightarrow \tilde{Y}$ of the universal covers, so that $\tilde{f}: C(\tilde{X}) \rightarrow C(\tilde{Y})$ is a chain equivalence of round finite $\mathbb{Z}[\pi]$-module chain complexes and torsion is defined as in Section 1, using the isomorphism $f_{*}: \pi_{1}(X)=\pi \rightarrow \pi_{1}(Y)$ as an identification. Any other lift of $f$ is given by

$$
\tilde{f} g: \tilde{X} \stackrel{g}{\longrightarrow} \tilde{X} \stackrel{\tilde{f}}{\longrightarrow} \tilde{Y}
$$

for some $g \in \pi$, and

$$
\begin{aligned}
\tau(\tilde{f g}: C(\tilde{X}) \rightarrow C(\tilde{Y})) & =\tau(g: C(\tilde{X}) \rightarrow C(\tilde{X}))+\tau(\tilde{f}: C(\tilde{X}) \rightarrow C(\tilde{Y})) \\
& =\tau\left(g^{\gamma(X)}: \mathbb{Z}[\pi] \rightarrow \mathbb{Z}[\pi]\right)+\tau(f) \\
& =\tau(f) \in K_{1}(\mathbb{Z}[\pi]) .
\end{aligned}
$$

Thus the torsion $\tau(f) \in K_{1}(\mathbb{Z}[\pi])$ is independent of the choice of lift $\tilde{f}: \tilde{X} \rightarrow \tilde{Y}$.

By the logarithmic property of torsion (Proposition 1.4(i)) the torsion of the composite $g f: X \rightarrow Z$ of homotopy equivalences $f: X \rightarrow Y, g: Y \rightarrow Z$ of round finite $\mathrm{CW}$ complexes is given by

$$
\tau(g f)=\tau(f)+\tau(g) \in K_{1}(\mathbb{Z}[\pi]),
$$

using the isomorphisms $f_{*}: \pi_{1}(X)=\pi \rightarrow \pi_{1}(Y), g_{*}: \pi_{1}(Y) \rightarrow \pi_{1}(Z)$ as identifications.

If $X ; X^{\prime}$ are round finite $\mathrm{CW}$ complexes with the same underlying $\mathrm{CW}$ complex the identity map has torsion

$$
\tau\left(1: X \rightarrow X^{\prime}\right) \in\{ \pm \pi\} \subset K_{1}(\mathbb{Z}[\pi]),
$$

measuring the difference between the two geometric bases. Thus, the image of $\tau(f: X \rightarrow Y) \in K_{1}(\mathbb{Z}[\pi])$ in $\mathrm{Wh}(\pi)$ is just the usual Whitehead torsion $\tau(f) \in \mathrm{Wh}(\pi)$.

A round finite structure on a CW complex $X$ is an equivalence class of pairs

(round finite $\mathrm{CW}$ complex $F$, homotopy equivalence $f: F \rightarrow X$ ) under the equivalence relation

$$
(F, f) \sim\left(F^{\prime}, f^{\prime}\right) \quad \text { if } \tau\left(f^{\prime-1} f: F \rightarrow F^{\prime}\right)=0 \in K_{1}(\mathbb{Z}[\pi]) \quad\left(\pi=\pi_{1}(X)\right) .
$$

The round finite structure set $\mathscr{F}^{r}(X)$ of a CW complex $X$ is the set (possibly empty) of round finite structures on $X$.

PROPOSITION 4.4. (i) $\mathscr{F}^{r}(X)$ is nonempty if and only if $X$ is finitely dominated and $[X]=0 \in K_{0}(\mathbb{Z}[\pi])$. 
(ii) If $\mathscr{F}^{r}(X)$ is nonempty there is defined a transitive $K_{1}(\mathbb{Z}[\pi])$-action

$$
\begin{aligned}
& K_{1}(\mathbb{Z}[\pi]) \times \mathscr{F}^{r}(X) \rightarrow \mathscr{F}^{r}(X) ; \\
& \quad(\tau(g: G \rightarrow F),(F, f)) \rightarrow(G, f g: G \rightarrow X) .
\end{aligned}
$$

A choice of base point $\left(F_{0}, f_{0}\right) \in \mathscr{F}^{r}(X)$ determines an Abelian group structure on $\mathscr{F}^{r}(X)$ with an isomorphism

$$
\mathscr{F}^{r}(X) \rightarrow K_{1}(\mathbb{Z}[\pi]) ; \quad(F, f) \rightarrow \tau\left(f^{-1} f_{0}: F_{0} \rightarrow F\right) .
$$

(iii) $\mathscr{F}^{r}(X)$ is in natural bijective correspondence with the round finite structure set $\mathscr{F}^{r}(C(\tilde{X}))$ of the cellular $\mathbb{Z}[\pi]$-module chain complex $C(\bar{X})$. If the sets are nonempty there is defined a natural isomorphism of affine $K_{1}(\mathbb{Z}[\pi])$-sets

$$
\mathscr{F}^{r}(X) \rightarrow \mathscr{F}^{r}(C(\tilde{X})) ;(F, f: F \rightarrow X) \rightarrow(C(\tilde{F}), \tilde{f}: C(\tilde{F}) \rightarrow C(\tilde{X})) .
$$

The product $X \times Y$ of connected $\mathrm{CW}$ complexes $X, Y$ is a connected $\mathrm{CW}$ complex with fundamental group

$$
\pi_{1}(X \times Y)=\pi_{1}(X) \times \pi_{1}(Y),
$$

so that

$$
\mathbb{Z}\left[\pi_{1}(X \times Y)\right]=\mathbb{Z}\left[\pi_{1}(X)\right] \otimes \mathbb{Z}\left[\pi_{1}(Y)\right] .
$$

The universal cover of $X \times Y$ is the product $\bar{X} \times \bar{Y}$ of the universal covers $\tilde{X}, \bar{Y}$ of $X, Y$, with cellular chain complex over $\mathbb{Z}\left[\pi_{1}(X \times Y)\right]$

$$
C(\hat{X} \times \bar{Y})=C(\bar{X}) \otimes C(\bar{Y}) .
$$

The product formulae obtained for chain complexes in Section 3 above can thus be translated directly into product formulae for CW complexes.

PROPOSITION 4.5. (i) (Gersten [7], Siebenmann [18]) The product of finitely dominated $C W$ complexes $X, Y$ is a finitely dominated $C W$ complex $X \times Y$ with projective class

$$
[X \times Y]=[X] \otimes[Y] \in K_{0}\left(\mathbb{Z}\left[\pi_{1}(X \times Y)\right]\right) .
$$

(ii) (Kwun and Szczarba [10]) The Whitehead torsion of the product $f \times g: X \times Y \rightarrow X^{\prime} \times Y^{\prime}$ of homotopy equivalences of finite $C W$ complexes $f: X \rightarrow X^{\prime}, g: Y \rightarrow Y^{\prime}$ is given by

$$
\tau(f \times g)=\tau(f) \chi(Y)+\chi(X) \tau(g) \in \mathrm{Wh}\left(\pi_{1}(X \times Y)\right) .
$$

Proof. (i) Immediate from Proposition 4.1(i).

(ii) Immediate from Proposition 4.1(iv).

In particular, the product $X \times Y$ of a finitely dominated CW complex $X$ and a round finite $\mathrm{CW}$ complex $Y$ has projective class

$$
[X \times Y]=[X] \otimes[Y]=[X] \otimes \chi(Y)=[X] \otimes 0=0 \in K_{0}\left(\mathbb{Z}\left[\pi_{1}(X \times Y)\right]\right),
$$


so that $X \times Y$ is homotopy equivalent to a round finite $\mathrm{CW}$ complex. More precisely:

PROPOSITION 4.6. (i) The product $X \times Y$ of a finitely dominated $C W$ complex $X$ and a round finite $C W$ complex $Y$ has a canonical product round finite structure.

(ii) The product $f \times g: X \times Y \rightarrow X^{\prime} \times Y^{\prime}$ of a homotopy equivalence $f: X \rightarrow X^{\prime}$ of finitely dominated $C W$ complexes and a homotopy equivalence $g: Y \rightarrow Y^{\prime}$ of round finite $C W$ complexes is a homotopy equivalence of $C W$ complexes with canonical round finite structures. The torsion of $f \times g$ with respect to the canonical round finite structures is the product

$$
\tau(f \times g)=[X] \otimes \tau(g) \in K_{1}\left(\mathbb{Z}\left[\pi_{1}(X \times Y)\right]\right)
$$

of the projective class $[X]=\left[X^{\prime}\right] \in K_{0}\left(\mathbb{Z}\left[\pi_{1}(X)\right]\right)$ and the torsion $\tau(g) \in K_{1}\left(\mathbb{Z}\left[\pi_{1}(Y)\right]\right)$.

\section{Proof. Immediate from Propositions 3.2, 4.4.}

The case $Y=S^{1}$ of Proposition 4.6 is particularly interesting, and will be dealt with separately in Section 5 below.

In the special case when $f: X \rightarrow X^{\prime}$ is a homotopy equivalence of finite $\mathrm{CW}$ complexes the product formula of Proposition 4.6(ii) agrees with the product formula $\tau(f \times g)=\chi(X) \tau(g) \in \mathrm{Wh}\left(\pi_{1}(X \times Y)\right)$ given by Proposition 4.5 (ii).

In the special case when $f: X \rightarrow X=X^{\prime}, g: Y \rightarrow Y=Y^{\prime}$ are self-homotopy equivalences such that $f_{*}=1: \pi_{1}(X) \rightarrow \pi_{1}(X), g_{*}=1: \pi_{1}(Y) \rightarrow \pi_{1}(Y)$ the product formula of Proposition 4.6(ii) agrees with the product formula for the torsion of selfhomotopy equivalences obtained by Gersten [8], which we shall recall in Proposition 5.2 below.

Given a map $\phi: X \rightarrow Y$ of finitely dominated $\mathrm{CW}$ complexes, let $\alpha$ denote the induced morphism of fundamental groups

$$
\alpha=\phi_{*}: \pi_{1}(X) \rightarrow \pi_{1}(Y),
$$

and let $A=\mathbb{Z}\left[\pi_{1}(Y)\right]$, so that there is induced a chain map of finitely dominated chain complexes over $A$

$$
\tilde{\phi}: \alpha_{1} C(\hat{X}) \rightarrow C(\tilde{Y})
$$

with $\tilde{X}, \tilde{Y}$ the universal covers of $X, Y$. If $f: A \rightarrow B$ is a ring morphism such that $\phi: X \rightarrow Y$ is a $B$-coefficient homology equivalence, then by the construction of Section 2 there is defined an invariant

$$
(X, Y, \phi)=\left(\alpha_{1} C(\tilde{X}), C(\hat{Y}), f_{1} \tilde{\phi}\right) \in K_{1}(f)
$$

with image

$$
\partial(X, Y, \phi)=[Y]-\alpha_{1}[X] \in K_{0}(A) .
$$

If $\phi: X \rightarrow X=Y$ is such that $\alpha=1: \pi_{1}(X) \rightarrow \pi_{1}(X)=\pi_{1}(Y)$ there is defined an element $\tau\left(f_{!} \tilde{\phi}: f_{!} C(\tilde{X}) \rightarrow f_{!} C(\tilde{X})\right) \in K_{1}(B)$ (see Section 5 below for details) with image $j \tau\left(f_{1} \tilde{\phi}\right)=(X, X, \phi) \in K_{1}(f)$. 
EXAMPLE. Let $f: \mathbb{Z}\left[z, z^{-1}\right] \rightarrow P^{-1} \mathbb{Z}\left[z, z^{-1}\right]$ be the localization map inverting the multiplicative subset $P=\left\{p(z) \in \mathbb{Z}\left[z, z^{-1}\right] \mid p(1)= \pm 1 \in \mathbb{Z}\right\}$ of $\mathbb{Z}\left[z, z^{-1}\right]$. This has the property that a finite chain complex $C$ over $\mathbb{Z}\left[z, z^{-1}\right]$ is such that $f_{1} C=P^{-1} C$ is contractible if and only if $\mathbb{Z} \otimes_{\mathbb{Z}\left[z, z^{-1}\right]} C$ is contractible (see Proposition 7.9 .2 of Ranicki [14] for a proof). For any locally flat $n$-knot $k: S^{n} \subset S^{n+2}$ the knot complement

$$
X=\text { closure of }\left(S^{n+2} \text { - regular neighbourhood of } k\left(S^{n}\right)\right)
$$

is such that the generator $1 \in H^{1}(X)=\left[X, S^{1}\right]=\mathbb{Z}$ is represented by a $\mathbb{Z}$ coefficient homology equivalence $\phi: X \rightarrow S^{1}$. The element

$$
\left(X, S^{1}, \phi\right) \in K_{1}(f)=\operatorname{coker}\left(f_{!}: K_{1}\left(\mathbb{Z}\left[z, z^{-1}\right]\right) \rightarrow K_{1}\left(P^{-1} \mathbb{Z}\left[z, z^{-1}\right]\right)\right)
$$

is the Reidemeister torsion of the $\operatorname{knot} k$.

\section{The Torsion of a Self Equivalence}

We shall now compare the notion of torsion $\tau(f) \in K_{1}(A)$ defined in Section 1 for a chain equivalence $f: C \rightarrow D$ of round finite chain complexes over $A$ with the torsion $\tau(f) \in K_{1}(A)$ defined by Gersten [8] for a self-chain equivalence $f: C \rightarrow C$ of a finitely dominated chain complex $C$ over $A$. This was applied in [8] to define the absolute torsion $\tau(f) \in K_{1}\left(\mathbb{Z}\left[\pi_{1}(X)\right]\right)$ of a self-homotopy equivalence $f: X \rightarrow X$ of a finitely dominated CW complex $X$ such that $f_{*}=1: \pi_{1}(X) \rightarrow \pi_{1}(X)$. In Section 6 we shall need to deal with self-homotopy equivalences $f: X \rightarrow X$ (notably $-1: S^{1} \rightarrow S^{1}$ ) such that $f_{*} \neq 1$, so we shall consider the general case here.

In dealing with self-chain equivalences it is convenient to modify the sign conventions for the algebraic mapping cone. The modified algebraic mapping cone $\hat{C}(f)$ of an $A$-module chain map $f: C \rightarrow D$ is the $A$-module chain complex defined by

$$
\begin{aligned}
& d_{\hat{C}(f)}=\left(\begin{array}{cc}
-d_{C} & 0 \\
f & d_{D}
\end{array}\right): \\
& \hat{C}(f)_{r}=C_{r-1} \oplus D_{r} \rightarrow \hat{C}(f)_{r-1}=C_{r-2} \oplus D_{r-1} \quad(r \in \mathbb{Z}) .
\end{aligned}
$$

PROPOSITION 5.1. (i) The modified algebraic mapping cone $\hat{C}(f)$ of a chain equivalence $f: C \rightarrow D$ of finite chain complexes over $A$ is a contractible finite chain complex over $A$ such that

$$
\begin{aligned}
& \tau(\hat{C}(f))-\tau(C(f)) \\
& \quad=\left(\chi_{\text {odd }}(C)+\Sigma_{r} \operatorname{rank}_{A}\left(C_{r-1}\right) \operatorname{rank}_{A}\left(D_{r}\right)\right) \tau(-1: A \rightarrow A) \in K_{1}(A) .
\end{aligned}
$$

(ii) For any chain equivalences $f: C \rightarrow D, g: D \rightarrow E$ of finite chain complexes over $A$ the composite chain equivalence $g f: C \rightarrow E$ is such that

$$
\begin{aligned}
& \tau(\hat{C}(g f)) \\
& \quad=\tau(\hat{C}(f))+\tau(\hat{C}(g))+\beta(S C \oplus S D, D \oplus E) \tau(-1: A \rightarrow A) \in K_{1}(A) .
\end{aligned}
$$

(iii) For any chain equivalences $f: C \rightarrow D, f^{\prime}: C^{\prime} \rightarrow D^{\prime}$ of finite chain complexes over 
$A$ the sum chain equivalence $f \oplus f^{\prime}: C \oplus C^{\prime} \rightarrow D \oplus D^{\prime}$ is such that

$$
\begin{aligned}
\tau\left(\hat{C}\left(f \oplus f^{\prime}\right)\right) \\
= \\
\quad \tau\left(\hat{C}(f)+\tau\left(\hat{C}\left(f^{\prime}\right)\right)+\left(\beta\left(D \oplus S C, D^{\prime} \oplus S C^{\prime}\right)+\right.\right. \\
\left.\quad+\Sigma_{r} \operatorname{rank}_{A}\left(C_{r-1}^{\prime}\right) \operatorname{rank}_{A} D_{r}\right) \tau(-1: A \rightarrow A) \in K_{1}(A) .
\end{aligned}
$$

Proof. (i) Apply Proposition 2.2 of Part I to the isomorphism of contractible finite chain complexes

$$
g: C(f) \rightarrow \hat{C}(f)
$$

defined by

$$
g=\left(\begin{array}{cc}
0 & (-)^{r-1} \\
1 & 0
\end{array}\right): C(f)_{r}=D_{r} \oplus C_{r-1} \rightarrow \hat{C}(f)_{r}=C_{r-1} \oplus D_{r} \quad(r \geqslant 0) .
$$

(ii) and (iii) Translate the formulae of Proposition 1.2 (i) and (ii) using (i) above.

It follows from the formulae of Proposition 5.1 that for any finite chain complex $C$ over $A$

$$
\tau(\hat{C}(1: C \rightarrow C))=0 \in K_{1}(A),
$$

and that for any chain equivalence $f: C \rightarrow D$ of round finite chain complexes over $A$

$$
\tau(f)=\tau(\hat{C}(f))+\beta(S C, C \oplus D) \tau(-1: A \rightarrow A) \in K_{1}(A) .
$$

In particular, for a self-chain equivalence $f: C \rightarrow D=C$ of a round finite chain complex $C$ over $A$ the sign term vanishes and

$$
\tau(f)=\tau(\hat{C}(f)) \in K_{1}(A) .
$$

Following Gersten [8] define the torsion of a self-chain equivalence $f: C \rightarrow C$ of a finitely dominated chain complex $C$ over $A$ by

$$
\tau(f)=\tau(\widehat{C}(e)) \in K_{1}(A)
$$

with $e$ the composite self-chain equivalence of a finite chain complex $D$ over $A$ given by

$$
e: D \stackrel{i}{\longrightarrow} C \oplus C^{\prime} \stackrel{f^{-} \oplus 1}{\longrightarrow} C \oplus C^{\prime} \stackrel{i^{-1}}{\longrightarrow} D
$$

for any finite chain complex $D$ such that there exists a chain equivalence $i: D \rightarrow C \oplus C^{\prime}$ with $C^{\prime}$ a finitely dominated chain complex, and any such $i$. (For example, if $\left(D^{\prime}, f^{\prime}, g^{\prime}, h^{\prime}\right)$ is a finite domination of $C$, then $D=D^{\prime}$ is such a finite chain complex, with $C^{\prime}=C\left(f^{\prime}: C \rightarrow D\right)$ a finitely dominated chain complex and

$$
i=\left(\begin{array}{l}
g^{\prime} \\
e^{\prime}
\end{array}\right): D \rightarrow C \oplus C^{\prime}
$$


a chain equivalence, where $e^{\prime}: D \rightarrow C^{\prime}$ is the inclusion.) If $C$ is a finite chain complex it is possible to choose $C^{\prime}=0, i=1: D=C \rightarrow C$, so that $e=f: C \rightarrow C$ and

$$
\tau(f)=\tau(\hat{C}(f)) \in K_{1}(A) .
$$

Note that $\tau(f) \in K_{1}(A)$ is independent of the base in $C$. Also, if $C$ is round finite this is the torsion $\tau(f) \in K_{1}(A)$ previously defined in Section 1, by the argument above. The torsion of an automorphism $f: C \rightarrow C$ of a bounded f.g. projective chain complex $C$ over $A$ is given by

$$
\tau(f)=\sum_{r=0}^{\infty}(-)^{r} \tau\left(f: C_{r} \rightarrow C_{p}\right) \in K_{1}(A) .
$$

Still following [8] define the torsion of a self-homotopy equivalence $f: X \rightarrow X$ of a finitely dominated $\mathrm{CW}$ complex $X$ inducing $f_{*}=1: \pi_{1}(X) \rightarrow \pi_{1}(X)$ by

$$
\tau(f)=\tau(\tilde{f}: C(\tilde{X}) \rightarrow C(\tilde{X})) \in K_{1}\left(\mathbb{Z}\left[\pi_{1}(X)\right]\right),
$$

with $\tilde{f}: C(\tilde{X}) \rightarrow C(\tilde{X})$ the induced self-chain equivalence of the finitely dominated cellular chain complex $C(\tilde{X})$ over $\mathbb{Z}\left[\pi_{1}(X)\right]$ of the universal cover $\tilde{X}$.

PROPOSITION 5.2 (Gersten [8]). (i) The torsion of self chain equivalences of finitely dominated chain complexes over $A$ is logarithmic and additive, with

$\tau(g f: C \rightarrow C)=\tau(f: C \rightarrow C)+\tau(g: C \rightarrow C) \in K_{1}(A)$,

$\tau\left(f \oplus f^{\prime}: C \oplus C^{\prime} \rightarrow C \oplus C^{\prime}\right)=\tau(f: C \rightarrow C)+\tau\left(f^{\prime}: C^{\prime} \rightarrow C^{\prime}\right) \in K_{1}(A)$.

(ii) The product $f \otimes g: C \otimes D \rightarrow C \otimes D$ of self-chain equivalences $f: C \rightarrow C$, $g: D \rightarrow D$ of finitely dominated chain complexes $C, D$ over $A, B$ (respectively) is a selfchain equivalence of a finitely dominated chain complex $C \otimes D$ over $A \otimes B$ with torsion

$$
\tau(f \otimes g)=[C] \otimes \tau(g)+\tau(f) \otimes[D] \in K_{1}(A \otimes B) .
$$

(iii) The product $f \times g: X \times Y \rightarrow X \times Y$ of self-homotopy equivalences $f: X \rightarrow X$, $g: Y \rightarrow Y$ of finitely dominated $C W$ complexes $X, Y$ such that $f_{*}=1: \pi_{1}(X) \rightarrow \pi_{1}(X)$, $g_{*}=1: \pi_{1}(Y) \rightarrow \pi_{1}(Y)$ is a self homotopy equivalence of a finitely dominated $C W$ complex $X \times Y$ such that

$$
(f \times g)_{*}=f_{*} \times g_{*}=1: \pi_{1}(X \times Y)=\pi_{1}(X) \times \pi_{1}(Y) \rightarrow \pi_{1}(X) \times \pi_{1}(Y),
$$

with torsion

$$
\tau(f \otimes g)=[X] \otimes \tau(g)+\tau(f) \otimes[Y] \in K_{1}\left(\mathbb{Z}\left[\pi_{1}(X \times Y)\right]\right) .
$$

A self-homotopy equivalence $f: X \rightarrow X$ of a finitely dominated CW complex $X$ induces an automorphism of the fundamental group

$$
f_{*}=\alpha: \pi_{1}(X)=\pi \rightarrow \pi
$$

and, hence, a chain equivalence of finitely dominated chain complexes over $\mathbb{Z}[\pi]$

$$
\tilde{f}: \alpha_{1} C(\tilde{X}) \rightarrow C(\tilde{X}) .
$$

If $[X]=0 \in K_{0}(\mathbb{Z}[\pi])$ and there is given a round finite structure $(F, \phi) \in \mathscr{F} r(C(\tilde{X}))$ 
$\left(=\mathscr{F}^{r}(X)\right.$, by definition) there is defined a torsion

$$
\begin{aligned}
& \tau_{(F, \phi)}(f) \\
& \quad=\tau\left(\phi^{-1} \tilde{f} \alpha_{1} \phi: \alpha_{!} F \stackrel{\alpha_{!} \phi}{\longrightarrow} \alpha_{!} C(\tilde{X}) \stackrel{\tilde{f}}{\longrightarrow} C(\tilde{X}) \stackrel{\phi^{-1}}{\longrightarrow} F\right) \in K_{1}(\mathbb{Z}[\pi]) .
\end{aligned}
$$

However, if $\alpha \neq 1$ this will in general depend on the choice of round finite structure $(F, \phi)$ :

PROPOSITION 5.3. The torsions associated to two different round finite structures $(F, \phi),\left(F^{\prime}, \phi^{\prime}\right) \in \mathscr{F}^{r}(X)$ differ by

$$
\begin{aligned}
& \tau_{\left(F^{\prime}, \phi^{\prime}\right)}(f)-\tau_{(F, \phi)}(f) \\
& \quad=\left(1-\alpha_{1}\right) \tau\left(\phi^{\prime-1} \phi: F \rightarrow F^{\prime}\right) \in K_{1}(\mathbb{Z}[\pi]) .
\end{aligned}
$$

Proof. Consider the commutative diagram of chain complexes over $\mathbb{Z}[\pi]$ and chain equivalences

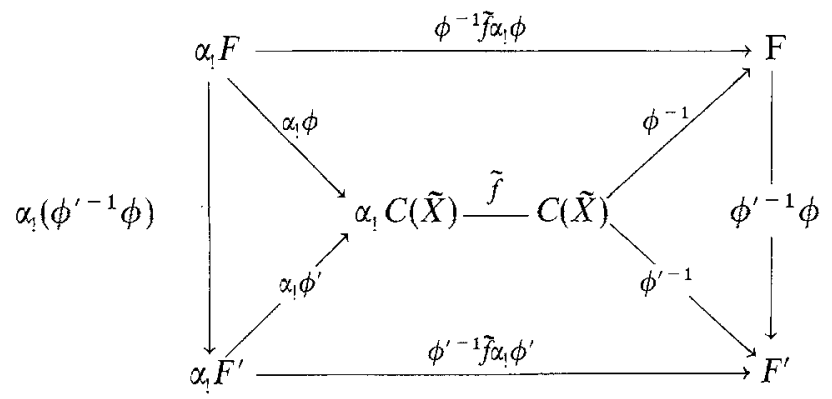

and apply the logarithmic property of torsion to the chain equivalences of round finite chain complexes on the outside.

Given a ring $A$ and an automorphism $\alpha: A \rightarrow A$ denote the relative $K_{1}$-group $K_{1}(1: A \rightarrow A, \alpha: A \rightarrow A)$ of Section 2 by $K_{1}(A, \alpha)$, so that there is defined an exact sequence

$$
K_{1}(A) \stackrel{1-\alpha_{1}}{\longrightarrow} K_{1}(A) \stackrel{j}{\longrightarrow} K_{1}(A, \alpha) \stackrel{\partial}{\longrightarrow} K_{0}(A) \stackrel{1-\alpha_{1}}{\longrightarrow} K_{0}(A) .
$$

$K_{1}(A, \alpha)$ is isomorphic to the relative $K_{1}$-group defined by Siebenmann [19].

Given a finitely dominated $\mathrm{CW}$ complex $X$ and a self-homotopy equivalence $f: X \rightarrow X$, let $\alpha: A \rightarrow A$ be the automorphism of the group ring $A=\mathbb{Z}\left[\pi_{1}(X)\right]$ induced by $f_{*}: \pi_{1}(X) \rightarrow \pi_{1}(X)$. Applying the construction of Section 2 to the induced chain equivalence of finitely dominated chain complexes over $A$

$$
\tilde{f}: \alpha_{1} C(\tilde{X}) \rightarrow C(\tilde{X})
$$


there is defined an element

$$
(X, f)=(C(\tilde{X}), \tilde{f}) \in K_{1}(A, \alpha)
$$

such that

$$
\partial(X, f)=[X] \in K_{0}(A) .
$$

If $[X]=0 \in K_{0}(A)$ a choice of round finite structure $(F, \phi) \in \mathscr{F}^{r}(X)=\mathscr{F}^{r}(C(\tilde{X}))$ determines an element $\tau_{(F, \phi)}(f) \in K_{1}(A)$ such that

$$
j \tau_{(F, \phi)}(f)=(X, f) \in K_{1}(A, \alpha) .
$$

Proposition 5.3 describes the effect on $\tau_{\{F, \phi\}}(f) \in K_{1}(A)$ of a different choice of round finite structure, in precise accordance with the identity

$$
\operatorname{im}\left(1-\alpha_{1}: K_{1}(A) \rightarrow K_{1}(A)\right)=\operatorname{ker}\left(j: K_{1}(A) \rightarrow K_{1}(A, \alpha)\right)
$$

given by the above exact sequence.

For $\alpha=1: A \rightarrow A$ there is defined a natural isomorphism

$$
K_{1}(A, 1) \rightarrow K_{1}(A) \oplus K_{0}(A) ; \quad(P, Q,[\phi]: P \oplus Q \rightarrow P \oplus Q) \rightarrow(\tau([\phi]),[P]-[Q])
$$

If $f: X \rightarrow X$ is a self homotopy equivalence of a finitely dominated CW complex $X$ such that $f_{*}=1: \pi_{1}(X) \rightarrow \pi_{1}(X)$ and $A=\mathbb{Z}\left[\pi_{1}(X)\right]$ the element $(X, f) \in K_{1}(A, 1)$ has image $(\tau(f),[X]) \in K_{1}(A) \oplus K_{0}(A)$ under this isomorphism, with $\tau(f) \in K_{1}(A)$ the torsion defined by Gersten [8].

The circle $S^{1}=[0,1] /(0=1)=e^{0} \cup e^{1}$ is a finite $\mathrm{CW}$ complex such that $\chi\left(S^{1}\right)=0 \in \mathbb{Z}$, with fundamental group $\pi_{1}\left(S^{1}\right)=\mathbb{Z}$ and universal cover $\widetilde{S}^{1}=\mathbb{R}$. Let $z$ be the generator

$$
z=\left(1: S^{1} \rightarrow S^{1}\right) \in \pi_{1}\left(S^{1}\right)
$$

so that $\pi_{1}\left(S^{1}\right)=\left\{z^{n} \mid n \in \mathbb{Z}\right\}$ and there is a natural identification of $\mathbb{Z}\left[\pi_{1}\left(S^{1}\right)\right]$ with the Laurent polynomial extension ring of $\mathbb{Z}$

$$
\mathbb{Z}\left[\pi_{1}\left(S^{1}\right)\right]=\mathbb{Z}\left[z, z^{-1}\right] .
$$

Define the canonical round finite structure $\Sigma^{1}=(D, \omega) \in \mathscr{F}^{r}\left(S^{1}\right)$ by $\omega=1$ : $D=C\left(\tilde{S}^{1}\right) \rightarrow C\left(\tilde{S}^{1}\right)$, with

$$
D=C\left(\tilde{S}^{1}\right): \mathbb{Z}\left[z, z^{-1}\right] \stackrel{1-z}{\longrightarrow} \mathbb{Z}\left[z, z^{-1}\right] .
$$

The geometric base elements are oriented lifts $\tilde{e}^{0}, \tilde{e}^{1} \subset \tilde{S}^{1}$ of the cells $e^{0}, e^{1} \subset S^{1}$ such that $\tilde{e}^{0} \subset \tilde{e}^{1}$.

The tensor product of a ring $A$ and $\mathbb{Z}\left[z, z^{-1}\right]$ is the Laurent polynomial ring of $A$

$$
A \otimes \mathbb{Z}\left[z, z^{-1}\right]=A\left[z, z^{-1}\right]
$$

The tensor product of a chain complex $C$ over $A$ and $D=C\left(\tilde{S}^{1}\right)$ is the modified 
algebraic mapping cone chain complex over $A\left[z, z^{-1}\right]$

$C \otimes D=\hat{C}\left(1-z: C\left[z, z^{-1}\right] \rightarrow C\left[z, z^{-1}\right]\right)$.

For finite $C$ this is an identity of round finite chain complexes. For finitely dominated $C$ Proposition 3.2 gives the canonical product round finite structure

$C \otimes \Sigma^{1}=(F, \phi) \in \mathscr{F}^{r}(C \otimes D)$

as defined by

$\phi: F=(E, p) \otimes D \stackrel{\text { projection }}{\longrightarrow} \operatorname{im}(p) \otimes D \stackrel{\theta \otimes 1}{\longrightarrow} C \otimes D$

for any projection $p=p^{2}: E \rightarrow E$ of an even finite chain complex $E$ over $A$ with a chain equivalence $\theta: \operatorname{im}(p) \leadsto C$, and with

$$
\begin{aligned}
d_{F}= & \left(\begin{array}{cc}
-d_{E} \otimes 1 & 0 \\
p \otimes d_{D}+(1-p) \otimes d_{\Delta} & d_{E} \otimes 1
\end{array}\right): \\
& F_{r+1}=E_{r} \otimes D_{1} \otimes E_{r+1} \otimes D_{0} \rightarrow F_{r}=E_{r-1} \otimes D_{1} \otimes E_{r} \otimes D_{0}
\end{aligned}
$$

for any differential $d_{\Delta} \in \operatorname{Hom}_{\bar{z}\left[z, z^{-1}\right]}\left(D_{1}, D_{0}\right)$ such that $D_{\Delta}$ is a contractible finite chain complex over $\mathbb{Z}\left[z, z^{-1}\right]$ with

$$
\tau\left(\mathbf{D}_{\Delta}\right)=0 \in K_{1}\left(\mathbb{Z}\left[z, z^{-1}\right]\right) \text {. }
$$

Making the obvious choice

$$
d_{\Delta}=1: D_{1}=\mathbb{Z}\left[z, z^{-1}\right] \rightarrow D_{0}=\mathbb{Z}\left[z, z^{-1}\right]
$$

note that

$$
\begin{aligned}
p & \otimes d_{D}+(1-p) \otimes d_{\Delta} \\
& =(1-z) p+(1-p) \\
& =1-z p \\
& : E_{r} \otimes D_{1}=E_{r}\left[z, z^{-1}\right] \rightarrow E_{\mathrm{r}} \otimes D_{0}=E_{r}\left[z, z^{-1}\right]
\end{aligned}
$$

and so

$$
F=\hat{C}\left(1-z p: E\left[z, z^{-1}\right] \rightarrow E\left[z, z^{-1}\right]\right),
$$

with

$$
\begin{aligned}
\phi & =\left(\begin{array}{ll}
\theta p & 0 \\
0 & \theta p
\end{array}\right): F_{r}=E_{r-1}\left[z, z^{-1}\right] \oplus E_{r}\left[z, z^{-1}\right] \rightarrow(C \otimes D)_{r} \\
& =C_{r-1}\left[z, z^{-1}\right] \oplus C_{r}\left[z, z^{-1}\right] .
\end{aligned}
$$

For any connected CW complex $X$ the product $\mathrm{CW}$ complex $X \times S^{1}$ has fundamental group

$$
\pi_{1}\left(X \times S^{1}\right)=\pi_{1}(X) \times \mathbb{Z}
$$


and there is a natural identification of rings

$$
\mathbb{Z}\left[\pi_{1}\left(X \times S^{1}\right)\right]=\mathbb{Z}\left[\pi_{1}(X)\right]\left[z, z^{-1}\right],
$$

so that the cellular chain complex of the universal cover $X \times S^{1}=\tilde{X} \times \tilde{S}^{1}$ can be expressed as

$$
\begin{aligned}
C\left(\widetilde{X \times S^{1}}\right) & =C\left(\tilde{X} \times \tilde{S}^{1}\right)=C(\tilde{X}) \otimes C\left(\tilde{S}^{1}\right) \\
& =\hat{C}\left(1-z: C(\tilde{X})\left[z, z^{-1}\right] \rightarrow C(\tilde{X})\left[z, z^{-1}\right]\right) .
\end{aligned}
$$

For finitely dominated $X$ define the canonical round finite structure on $X \times S^{1}$ by

$$
X \times \Sigma^{1}=C(\tilde{X}) \otimes(D, \omega) \in \mathscr{F}^{r}\left(X \times S^{1}\right)=\mathscr{F}^{r}\left(C\left(\widetilde{X \times S^{1}}\right)\right) .
$$

In Section 6 below we shall identify the reduction of $X \times \Sigma^{1}$ in $\mathscr{F}\left(X \times S^{1}\right)$ with the canonical finite structure defined geometrically on $X \times S^{1}$ by Mather [12] and Ferry [6].

The self-homeomorphism of $S^{1}=[0,1] /(0=1)$

$$
-1: S^{1} \rightarrow S^{1} ; \quad s \mapsto 1-s \quad(0 \leqslant s \leqslant 1)
$$

is such that

$$
\left(-1: S^{1} \rightarrow S^{1}\right)=z^{-1} \in \pi_{1}\left(S^{1}\right),
$$

and induces the automorphism

$$
(-1)_{*}=\alpha: \pi_{1}\left(S^{1}\right) \rightarrow \pi_{1}\left(S^{1}\right) ; \quad z^{n} \mapsto z^{-n} .
$$

PROPOSITION 5.8. (i) The torsion of $-1: S^{1} \rightarrow S^{1}$ with respect to the canonical round finite structure $\Sigma^{1} \in \mathscr{F}^{r}\left(S^{1}\right)$ is given by

$$
\tau_{\Sigma^{1}}(-1)=\tau\left(-z: \mathbb{Z}\left[z, z^{-1}\right] \rightarrow \mathbb{Z}\left[z, z^{-1}\right]\right) \in K_{1}\left(\mathbb{Z}\left[z, z^{-1}\right]\right) .
$$

(ii) If $X$ is a finitely dominated $C W$ complex the torsion of $1 \times-1: X \times S^{1} \rightarrow X \times S^{1}$ with respect to the canonical round finite structure $X \times \Sigma^{1} \in \mathscr{F}^{r}\left(X \times S^{1}\right)$ is given by

$$
\tau_{X \times \Sigma^{1}}(1 \times-1)=[X] \otimes \tau(-z) \in K_{1}\left(\mathbb{Z}\left[\pi_{1}(X)\right]\left[z, z^{-1}\right]\right) .
$$

Proof. (i) The induced chain equivalence $(\widetilde{-1}): \alpha_{1} D \rightarrow D$ is the isomorphism of round finite chain complexes over $\mathbb{Z}\left[z, z^{-1}\right]$

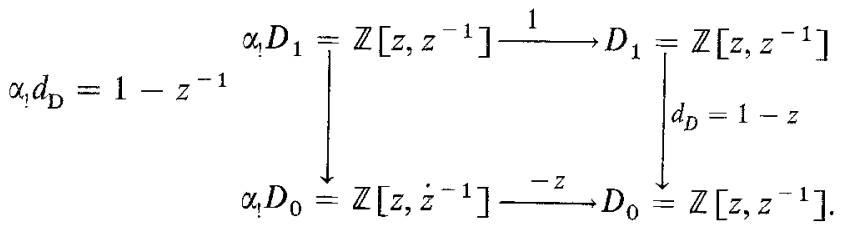


A direct application of Proposition 2.7 (iii) of Part I gives

$$
\begin{aligned}
& \tau_{\Sigma^{1}}\left(-1: S^{1} \rightarrow S^{1}\right) \\
& =\tau\left(-z: \mathbb{Z}\left[z, z^{-1}\right] \rightarrow \mathbb{Z}\left[z, z^{-1}\right]\right)-\tau\left(1: \mathbb{Z}\left[z, z^{-1}\right] \rightarrow \mathbb{Z}\left[z, z^{-1}\right]\right) \\
& =\tau(-z) \in K_{1}\left(\mathbb{Z}\left[z, z^{-1}\right]\right) .
\end{aligned}
$$

(ii) Substituting the result of (i) in the product formula of Proposition 4.6 (ii)

$$
\begin{aligned}
& \tau_{X \times \Sigma^{1}}\left(1 \times-1: X \times S^{1} \rightarrow X \times S^{1}\right) \\
& =[X] \otimes \tau_{\Sigma} 1\left(-1: S^{1} \rightarrow S^{1}\right) \\
& =[X] \otimes \tau(-z) \in K_{1}\left(\mathbb{Z}\left[\pi_{1}(X)\right]\left[z, z^{-1}\right]\right) .
\end{aligned}
$$

A noncanonical round finite structure $\left(D^{\prime}, \omega^{\prime}\right) \in \mathscr{F}^{r}\left(S^{1}\right)$ differs from the canonical structure $\Sigma^{1}=(D, \omega)$ by

$$
\begin{aligned}
& (D, \omega)-\left(D^{\prime}, \omega^{\prime}\right) \\
& \quad=\tau\left(\omega^{-1} \omega: D \rightarrow D^{\prime}\right) \in K_{1}\left(\mathbb{Z}\left[z, z^{-1}\right]\right) \\
& \quad=\left\{\tau\left( \pm z^{n}: \mathbb{Z}\left[z, z^{-1}\right] \rightarrow \mathbb{Z}\left[z, z^{-1}\right]\right) \mid n \in \mathbb{Z}\right\} \quad\left(=\mathbb{Z} \oplus \mathbb{Z}_{2}\right),
\end{aligned}
$$

say $(D, \omega)-\left(D^{\prime}, \omega^{\prime}\right)=\tau\left( \pm z^{n}\right)$. The torsion of $-1: S^{1} \rightarrow S^{1}$ with respect to $\left(D^{\prime}, \omega^{\prime}\right)$ is given by Propositions 5.3 and 5.4 to be

$$
\begin{aligned}
\tau_{\left(D^{\prime}, \omega^{\prime}\right)}(-1) & =\tau_{(D, \omega)}(-1)+\left(1-\alpha_{1}\right) \tau\left(\omega^{\prime-1} \omega\right) \\
& =\tau(-z)+\left(1-\alpha_{1}\right) \tau\left( \pm z^{n}\right) \\
& =\tau\left(-z^{2 n+1}\right) \in K_{1}\left(\mathbb{Z}\left[z, z^{-1}\right]\right) .
\end{aligned}
$$

It follows that for any finitely dominated CW complex $X$

$$
\begin{aligned}
\tau_{X \otimes\left(D^{\prime}, \omega^{\prime}\right)} & \left(1 \times-1: X \times S^{1} \rightarrow X \times S^{1}\right)- \\
& -\tau_{X \otimes(D, \omega)}\left(1 \times-1: X \times S^{1} \rightarrow X \times S^{1}\right) \\
= & {[X] \otimes\left(\tau_{\left(D^{\prime}, \omega^{\prime}\right)}(-1)-\tau_{(D, \omega)}(-1)\right) } \\
= & {[X] \otimes \tau\left(z^{2 n}\right) \in K_{1}\left(\mathbb{Z}\left[\pi_{1}(X)\right]\left[z, z^{-1}\right]\right) . }
\end{aligned}
$$

\section{The Mapping Torus in Algebra and Topology}

Actually, we shall start with the topology.

The mapping torus of a map $f: X \rightarrow X$ of a space $X$ to itself is the identification space

$$
T(f)=X \times[0,1] /\{(x, 0)=(f(x), 1) \mid x \in X\} .
$$

PROPOSITION 6.1. (i) A homotopy $e: f \simeq f^{\prime}: X \rightarrow X$ induces a homotopy equivalence

$$
S(e): T(f) \rightarrow T\left(f^{\prime}\right) .
$$


(ii) For any maps $f: X \rightarrow Y, g: Y \rightarrow X$ the maps

$$
\begin{aligned}
& S(f, g): T(g f: X \rightarrow X) \rightarrow T(f g: Y \rightarrow Y) ;(x, s) \mapsto(f(x), s) \\
& S(g, f): T(f g: Y \rightarrow Y) \rightarrow T(g f: X \rightarrow X) ;(y, t) \rightarrow(g(y), t)
\end{aligned}
$$

are inverse homotopy equivalences.

Proof. (i) Regard the mapping torus of $f: X \rightarrow X$ as the adjunction space

$$
T(f)=\left(X \times\left[0, \frac{1}{2}\right]\right) \cup_{g}\left(X \times\left[\frac{1}{2}, 1\right]\right),
$$

with the adjunction map defined by

$$
\begin{aligned}
& g: X \times\left\{0, \frac{1}{2}\right\} \rightarrow X \times\left[\frac{1}{2}, 1\right] ; \\
& (x, 0) \mapsto(f(x), 1), \quad\left(x, \frac{1}{2}\right) \mapsto\left(x, \frac{1}{2}\right) .
\end{aligned}
$$

A homotopy $e: f \simeq f^{\prime}: X \rightarrow X$ determines a homotopy of adjunction maps

$$
h: g \simeq g^{\prime}: X \times\left\{0, \frac{1}{2}\right\} \rightarrow X \times\left[\frac{1}{2}, 1\right]
$$

and, hence, a homotopy equivalence of the adjunction spaces

$$
\begin{aligned}
S(e) & =1 \cup_{h} 1: T(f) \\
& =\left(X \times\left[0, \frac{1}{2}\right]\right) \cup_{g}\left(X \times\left[\frac{1}{2}, 1\right]\right) \\
\simeq T\left(f^{\prime}\right) & =\left(X \times\left[0, \frac{1}{2}\right]\right) \cup_{g^{\prime}}\left(X \times\left[\frac{1}{2}, 1\right]\right),
\end{aligned}
$$

since the pair $\left(X \times\left[0, \frac{1}{2}\right], X \times\left\{0, \frac{1}{2}\right\}\right)$ has the homotopy extension property. There is no direct formula for $S(e)$, which is only determined up to homotopy.

(ii) Given a map $f: X \rightarrow X$ define a map

$$
U(f): T(f) \rightarrow T(f) ; \quad[x, t] \mapsto[f(x), t]
$$

and a homotopy

$$
e: U(f) \simeq 1: T(f) \rightarrow T(f)
$$

by

$$
\begin{aligned}
& e: T(f) \times I \rightarrow T(f) ; \quad([x, s], t) \mapsto \\
&\left\{\begin{array}{l}
{[f(x), s+t] \quad \text { if } \quad s+t \leqslant 1} \\
{[x, s+t-1] \quad \text { if } \quad s+t \geqslant 1}
\end{array}\right. \\
&(s, t \in I=[0,1]) .
\end{aligned}
$$

Now for any maps $f: X \rightarrow Y, g: Y \rightarrow X$ the composites of

$$
S(f, g): T(g f) \rightarrow T(f g), \quad S(g, f): T(f g) \rightarrow T(g f)
$$

are given by

$$
\begin{aligned}
& S(f, g) S(g, f)=U(f g): T(f g) \rightarrow T(f g) \\
& S(g, f) S(f, g)=U(g f): T(g f) \rightarrow T(g f),
\end{aligned}
$$


so that $S(f, g)$ and $S(g, f)$ are inverse homotopy equivalences.

We shall only be concerned with the mapping torus $T(f)$ when $X$ is a $\mathrm{CW}$ complex and $f: X \rightarrow X$ is a cellular map, so that $T(f)$ is a CW complex with two $r$ cells $e^{r} \times\{0\}, e^{r} \times\left\{\frac{1}{2}\right\}$ and two $(r+1)$-cells $e^{r} \times\left[0, \frac{1}{2}\right], e^{r} \times\left[\frac{1}{2}, 1\right]$ for each $r$-cell $e^{r}$ of $X$. If $X$ is a finite CW complex, then $T(f)$ is a finite CW complex such that $\chi(T(f))=0 \in \mathbb{Z}$, and so admits a round finite structure. We shall show that for any (cellular) map $f: X \rightarrow X$ of a finitely dominated CW complex $X$ the mapping torus $T(f)$ has a canonical round finite structure.

PROPOSITION 6.2. (i) For a finite CW complex $X$ a homotopy $e: f \simeq f^{\prime}: X \rightarrow X$ induces a homotopy equivalence $S(e): T(f) \rightarrow T\left(f^{\prime}\right)$ of finite $C W$ complexes which is simple, that is

$$
\tau(S(e))=0 \in \mathrm{Wh}\left(\pi_{1}(T(f)) .\right.
$$

(ii) For finite $C W$ complexes $X, Y$ and maps $f: X \rightarrow Y, g: Y \rightarrow X$ the homotopy equivalence $S(f, g): T(g f) \rightarrow T(f g)$ of finite $C W$ complexes is simple, that is

$$
\tau(S(f, g))=0 \in \mathrm{Wh}\left(\pi_{1}(T(g f))\right) .
$$

Proof. This may be deduced from the material on mapping cylinders and deformations in Section 5 of Cohen [4].

Given a finitely dominated $\mathrm{CW}$ complex $X$ and a map $\zeta: X \rightarrow X$ define a finite structure $(T(f \zeta g), \phi) \in \mathscr{F}(T(\zeta))$ for any finite domination $(Y, f: X \rightarrow Y$, $g: Y \rightarrow X, h: g f \simeq 1: X \rightarrow X)$ of $X$ by

$$
\begin{aligned}
\phi= & S(\zeta h) S(\zeta g, f): \\
& T(f \zeta g: Y \rightarrow Y) \rightarrow T(\zeta g f: X \rightarrow X) \rightarrow T(\zeta: X \rightarrow X) .
\end{aligned}
$$

PROPOSITION 6.3. The finite structure $(T(f \zeta g), \phi) \in \mathscr{F}(T(\zeta))$ is independent of the choice of finite domination $(Y, f, g, h)$ of $X$.

Proof. The finite structures $\left.(T(f \zeta \mathrm{g}), \phi), T\left(f^{\prime} \zeta g^{\prime}\right), \phi^{\prime}\right)$ on $T(\zeta)$ determined by any two finite dominations $(Y, f, g, h),\left(Y^{\prime}, f^{\prime}, g^{\prime}, h^{\prime}\right)$ of $X$ are such that up to homotopy

$$
\begin{aligned}
\phi^{\prime-1} \phi= & S\left(f^{\prime} \zeta h g^{\prime}\right)^{-1} S\left(f^{\prime} \zeta g, f g^{\prime}\right) S\left(f h^{\prime} \zeta g\right): \\
& T(f \zeta g) \rightarrow T\left(f g^{\prime} f^{\prime} \zeta g\right) \rightarrow T\left(f^{\prime} \zeta g f g^{\prime}\right) \rightarrow T\left(f^{\prime} \zeta g^{\prime}\right)
\end{aligned}
$$

a composite of simple homotopy equivalences by Proposition 6.2. It follows that $\tau\left(\phi^{\prime-1} \phi\right)=0 \in \mathrm{Wh}\left(\pi_{1}(T(\zeta))\right)$, and so

$$
(T(f \zeta g), \phi)=\left(T\left(f^{\prime} \zeta g^{\prime}\right), \phi^{\prime}\right) \in \mathscr{F}(T(\zeta))
$$

Call $(T(f \zeta g), \phi) \in \mathscr{F}(T(\zeta))$ the canonical finite structure on $T(\zeta)$. In the case $\zeta=1: X \rightarrow X$ this is the finite structure on $T(1)=X \times S^{1}$ defined by Mather [12] and Ferry [6]. 
EXAMPLE. Let $M$ be a compact $n$-manifold with a finitely dominated infinite cyclic cover $\bar{M}$, and let $\zeta: \bar{M} \rightarrow \bar{M}$ be a generating covering translation. Then the projection $p: \bar{M} \rightarrow M$ induces a homotopy equivalence of $\mathrm{CW}$ complexes with finite structure

$$
q: T(\zeta) \rightarrow M ; \quad(x, s) \mapsto p(x)
$$

such that $\tau(q) \in \mathrm{Wh}\left(\pi_{1}(M)\right)$ is the obstruction of Farrell [5] and Siebenmann [19] to fibering $M$ over $S^{1}$ (assuming $\mathrm{n} \geqslant 6$ ).

In order to compare the geometrically defined canonical finite structure on $X \times S^{1}$ with the algebraically defined canonical round finite structure of Section 5 , we shall use the following algebraic analogue of the mapping torus.

Given a ring $A$ and a morphism $\alpha: A \rightarrow A$ define the $\alpha$-twisted polynomial ring of $A, A *{ }_{\alpha}\left[z, z^{-1}\right]$ to be the quotient ring of the free product $A * \mathbb{Z}\left[z, z^{-1}\right]$ given by

$$
A *_{\alpha}\left[z, z^{-1}\right]=A * \mathbb{Z}\left[z, z^{-1}\right] /\left\{z^{-1} a z=\alpha(a) \mid a \varepsilon A\right\} .
$$

There is defined a morphism of rings

$$
i: A \rightarrow A *{ }_{\alpha}\left[z, z^{-1}\right] ; \quad a \mapsto a
$$

under which $\alpha$ becomes conjugation by $z$, which is injective if and only if $\alpha$ is injective. If $\alpha: A \rightarrow A$ is an automorphism $A *_{\alpha}\left[z, z^{-1}\right]=A_{\alpha}\left[z, z^{-1}\right]$ is the usual $\alpha$ twisted polynomial extension ring of $A$, which in the untwisted case $\alpha=1: A \rightarrow A$ is the Laurent polynomial extension $\operatorname{ring} A\left[z, z^{-1}\right]$.

Let then $A$ be a ring, $\alpha: A \rightarrow A$ a ring morphism, and for some chain complex $C$ over $A$ let $f: \alpha_{1} C \rightarrow C$ be a chain map. The algebraic mapping torus of $f$ is the chain complex over $A *_{\alpha}\left[z, z^{-1}\right]$ defined by

$$
T(f)=\hat{C}\left(1-z f: i_{!} C \rightarrow i_{!} C\right),
$$

using the modified algebraic mapping cone $\hat{C}$ of Section 5

$$
\begin{aligned}
d_{T(f)} & =\left(\begin{array}{cc}
-d & 0 \\
(1-z f) & d
\end{array}\right): T(f)_{r}=i_{1} C_{r-1} \oplus i_{i} C_{r} \rightarrow T(f)_{r-1} \\
& =i_{1} C_{r-2} \oplus i_{1} C_{r-1} .
\end{aligned}
$$

If $C$ is finite $T(f)$ is round finite. If $\alpha=1: A \rightarrow A$ there are natural identifications

$$
A *_{\alpha}\left[z, z^{-1}\right]=A\left[z, z^{-1}\right]=A \otimes \mathbb{Z}\left[z, z^{-1}\right]
$$

and for any chain complex $C$ over $A$

$$
T(1: C \rightarrow C)=C \otimes C\left(\widetilde{S}^{1}\right),
$$

which for finite $C$ is an identity of round finite chain complexes over $A\left[z, z^{-1}\right]$, using the canonical structure $\Sigma^{1} \in \mathscr{F}^{r}\left(C\left(\tilde{S}^{1}\right)\right)$. 
By analogy with Propositions 6.1, 6.2

PROPOSITION 6.4. (i) A chain homotopy e: $\mathrm{f} \simeq \mathrm{f}^{\prime}: \alpha_{1} \mathrm{C} \rightarrow \mathrm{C}$ induces an isomorphism of the algebraic mapping tori

$S(e): T(f) \rightarrow T\left(f^{\prime}\right)$.

For finite $C$

$$
\tau(S(e))=0 \in K_{1}\left(A *_{\alpha}\left[z, z^{-1}\right]\right)
$$

(ii) Let $\alpha: A \rightarrow B, \beta: B \rightarrow A$ be morphisms of rings, and let $f: \alpha_{1} C \rightarrow D, g: \beta_{1} D \rightarrow C$ be chain maps for some chain complexes $C, D$ over $A, B$ respectively. Then there are defined an isomorphism of rings

$$
k: A *_{\beta \alpha}\left[z, z^{-1}\right] \rightarrow B *_{\alpha \beta}\left[z, z^{-1}\right]
$$

and a chain equivalence of chain complexes over $B *_{\alpha \beta}\left[z, z^{-1}\right]$

$$
\begin{aligned}
S(f, g): & k_{!} T\left(g \beta_{!} f:(\beta \alpha)_{!} C \rightarrow C\right) \\
& \rightarrow T\left(f \alpha_{!} g:(\alpha \beta)_{!} D \rightarrow D\right) .
\end{aligned}
$$

If $C, D$ are finite

$$
\tau(S(f, g))=0 \in K_{1}\left(B *_{\alpha \beta}\left[z, z^{-1}\right]\right) .
$$

Proof. (i) The isomorphism $S(e): T(f) \rightarrow T\left(f^{\prime}\right)$ is defined by

$$
\begin{aligned}
S(e)= & \left(\begin{array}{ll}
1 & 0 \\
z e & 1
\end{array}\right): \\
& T(f)_{r}=i_{1} C_{r-1} \oplus i_{!} C_{r} \rightarrow T\left(f^{\prime}\right)_{r}=i_{!} C_{r-1} \oplus i_{1} C_{r} .
\end{aligned}
$$

(ii) Let

$$
i: A \rightarrow A *_{\beta \alpha}\left[z, z^{-1}\right], \quad j: B \rightarrow B *_{\alpha \beta}\left[z, z^{-1}\right]
$$

be the canonical ring morphisms. The isomorphism of polynomial rings

$$
k: A *_{\beta \alpha}\left[z, z^{-1}\right] \rightarrow B *_{\alpha \beta}\left[z, z^{-1}\right] ; \quad a \mapsto \alpha(a), z \mapsto z
$$

has inverse

$$
k^{-1}: B *_{\alpha \beta}\left[z, z^{-1}\right] \rightarrow A *_{\beta \alpha}\left[z, z^{-1}\right] ; \quad b \mapsto z \beta(b) z^{-1}, z \mapsto z,
$$

and there is defined a commutative square of rings and morphisms

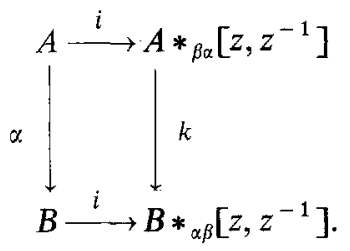


THE ALGEBRAIC THEORY OF TORSION. II: PRODUCTS

The chain maps of chain complexes over $B *_{\alpha \beta}\left[z, z^{-1}\right]$

$$
\begin{aligned}
& S(f, g): k_{1} T\left(g \beta_{1} f\right) \rightarrow T\left(f \alpha_{1} g\right) \\
& S^{\prime}(g, f): T\left(f \alpha_{1} g\right) \rightarrow k_{1} T\left(g \beta_{!} f\right)
\end{aligned}
$$

defined by

$$
\begin{aligned}
S(f, g) & =\left(\begin{array}{ll}
f & 0 \\
0 & f
\end{array}\right): k_{1} T\left(g \beta_{!} f\right)_{r} \\
& =j_{!} \alpha_{!} C_{r-1} \oplus j_{1} \alpha_{!} C_{r} \rightarrow T\left(f \alpha_{!} g\right)_{r} \\
& =j_{!} D_{r-1} \oplus j_{!} D_{r} \\
S^{\prime}(g, f) & =\left(\begin{array}{cc}
z \alpha_{!} g & 0 \\
0 & z \alpha_{!} g
\end{array}\right): T\left(f \alpha_{!} g\right)_{r} \\
& =j_{!} D_{r-1} \oplus j_{!} D_{r} \rightarrow k_{!} T\left(g \beta_{!} f\right)_{r} \\
& =j_{!} \alpha_{!} C_{r-1} \oplus j_{!} \alpha_{!} C_{r}
\end{aligned}
$$

are chain homotopy inverses, with chain homotopies

$$
\begin{aligned}
e & =\left(\begin{array}{ll}
0 & 1 \\
0 & 0
\end{array}\right): T\left(g \beta_{1} f\right)_{r} \\
& =j_{!} \alpha_{!} C_{r-1} \oplus j_{!} \alpha_{1} C_{r} \rightarrow j_{!} \alpha_{!} C_{r} \oplus j ! \alpha_{!} C_{r+1} \\
e^{\prime} & =\left(\begin{array}{ll}
0 & 1 \\
0 & 0
\end{array}\right): T\left(f \alpha_{1} g\right)_{r} \\
& =j_{!} D_{r-1} \oplus j_{!} D_{r} \rightarrow j_{!} D_{r} \oplus j_{!} D_{r+1} .
\end{aligned}
$$

Define a chain contraction of $C(S(f, g))$

$$
\Gamma: 0 \simeq 1: C(S(f, g)) \rightarrow C(S(f, g))
$$

by

$$
\begin{aligned}
\Gamma & =\left(\begin{array}{cc}
e^{\prime} & 0 \\
(-)^{r} S^{\prime}(g, f) & e
\end{array}\right): C(S(f, g))_{r} \\
& =T\left(f \alpha_{1} g\right)_{r} \oplus k_{!} T\left(g \beta_{1} f\right)_{r-1} \rightarrow T\left(f \alpha_{9} g\right)_{r+1} \oplus k_{!} T\left(g \beta_{!} f\right)_{r} .
\end{aligned}
$$

Thus, if $C, D$ are finite $S(f, g): k_{1} T\left(g \beta_{1} f\right) \rightarrow T\left(f \alpha_{1} g\right)$ is a chain equivalence of round finite chain complexes over $B *_{\alpha \beta}\left[z, z^{-1}\right]$ with torsion

$$
\begin{aligned}
\tau(S(f, g)) & =\tau(C(S(f, g))) \\
& =\tau\left(d+\Gamma: C(S(f, g))_{\mathrm{odd}} \rightarrow C(S(f, g))_{\mathrm{even}}\right)
\end{aligned}
$$




$$
\begin{aligned}
& =\tau\left(\left(\begin{array}{cc}
d^{\prime}+e^{\prime} & S(f, g) \\
-S^{\prime}(g, f) & d+e
\end{array}\right): T\left(f \alpha_{1} g\right)_{\text {odd }} \oplus k_{!} T\left(g \beta_{!} f\right)_{\text {even }}\right. \\
& \left.\rightarrow T\left(\dot{f} \alpha_{!} g\right)_{\text {even }} \oplus k_{!} T\left(g \beta_{!} f\right)_{\text {odd }}\right) \\
& =\tau\left(\begin{array}{cccc}
1 & -d & f & 0 \\
d & 1-z f \alpha_{1} g & 0 & f \\
-z \alpha_{1} g & 0 & 1-z g \beta_{!} f & d \\
0 & -z \alpha_{1} g & -d & 1
\end{array}\right) \\
& =\left(\begin{array}{cccc}
1 & 0 & 0 & 0 \\
d & 1 & 0 & 0 \\
-z \alpha_{1} g & 0 & 1 & 0 \\
0 & 0 & 0 & 1
\end{array}\right)\left(\begin{array}{llll}
1 & 0 & 0 & 0 \\
0 & 1 & 0 & f \\
0 & 0 & 1 & d \\
0 & 0 & 0 & 1
\end{array}\right) \\
& \left(\begin{array}{cccc}
1 & 0 & 0 & 0 \\
0 & 1 & 0 & 0 \\
0 & 0 & 1 & 0 \\
0 & -z \alpha_{1} g & -d & 1
\end{array}\right)\left(\begin{array}{cccc}
1 & -d & f & 0 \\
0 & 1 & 0 & 0 \\
0 & 0 & 1 & 0 \\
0 & 0 & 0 & 1
\end{array}\right) . \\
& : j_{1} D_{\text {odd }} \oplus j_{1} D_{\text {even }} \oplus j_{1} \alpha_{1} C_{\text {odd }} \oplus j_{1} \alpha_{1} C_{\text {even }} \\
& \left.\rightarrow j_{!} D_{\text {odd }} \oplus j_{!} D_{\text {even }} \oplus j_{1} \alpha_{!} C_{\text {odd }} \oplus j_{!} \alpha_{!} C_{\text {even }}\right) \\
& =0 \in K_{1}\left(B_{\alpha \beta \beta}^{*}\left[z, z^{-1}\right]\right) \text {. }
\end{aligned}
$$

Given a finitely dominated chain complex $C$ over a ring $A$ and a chain map $\zeta: \alpha_{1} C \rightarrow C$ for some morphism $\alpha: A \rightarrow A$ define a round finite structure $(T(f \zeta \alpha, g), \phi) \in \mathscr{F} r(T(\zeta))$ for any finite domination $(D, f: C \rightarrow D, g: D \rightarrow C, h: g f \simeq 1: C \rightarrow C)$ of $C$ by

$$
\begin{aligned}
\phi= & S\left(\zeta \alpha_{1} h\right) S\left(\zeta \alpha_{1} g, f\right): \\
& T\left(f \zeta \alpha_{1} g: \alpha_{1} D \rightarrow D\right) \rightarrow T\left(\zeta \alpha_{!}(g f): \alpha_{1} C \rightarrow C\right) \\
& \rightarrow T\left(\zeta: \alpha_{!} C \rightarrow C\right) .
\end{aligned}
$$

The round finite structures $\left(T\left(f \zeta \alpha_{1} g\right), \phi\right),\left(T\left(f^{\prime} \zeta \alpha_{1} g^{\prime}\right), \phi^{\prime}\right) \in \mathscr{F}^{\mathrm{F}}(T(\zeta))$ determined by two finite dominations $(D, f, g, h),\left(D^{\prime}, f^{\prime}, g^{\prime}, h^{\prime}\right)$ of $C$ are such that up to chain homotopy

$$
\begin{aligned}
\phi^{\prime-1} \phi= & S\left(f^{\prime} \zeta \alpha_{!}\left(h g^{\prime}\right)\right)^{-1} S\left(f^{\prime} \zeta \alpha_{1} g, f g^{\prime}\right) S\left(f h^{\prime} \zeta \alpha_{1} g\right): \\
& T\left(f \zeta \alpha_{1} g\right) \rightarrow T\left(f g^{\prime} f^{\prime} \zeta \alpha_{4} g\right) \rightarrow T\left(f^{\prime} \zeta \alpha_{1}\left(g f g^{\prime}\right)\right) \\
& \rightarrow T\left(f^{\prime} \zeta \alpha_{1} g^{\prime}\right),
\end{aligned}
$$


a composite of chain equivalences with $\tau=0 \in K_{1}\left(A *_{\alpha}\left[z, z^{-1}\right]\right)$ by Proposition 6.4, and so by analogy with Proposition 6.3

$$
\begin{aligned}
& \left(T\left(f \zeta \alpha_{!} g\right), \phi\right)-\left(T\left(f^{\prime} \zeta \alpha_{1} g^{\prime}\right), \phi^{\prime}\right) \\
& \quad=\tau\left(\phi^{\prime-1} \phi\right)=0 \in K_{1}\left(A *_{\alpha}\left[z, z^{-1}\right]\right) .
\end{aligned}
$$

Thus the round finite structure

$$
\left(T\left(f \zeta \alpha_{1} g\right), \phi\right)=\left(T\left(f^{\prime} \zeta \alpha_{1} g^{\prime}\right), \phi^{\prime}\right) \in \mathscr{F}^{r}(T(\zeta))
$$

is independent of the finite domination of $C$; we shall call this the canonical round finite structure on $T(\zeta)$. In particular, for $\alpha=1: A \rightarrow A, \zeta=1: \alpha_{1} C=C \rightarrow C$ we have: PROPOSITION 6.5. The canonical round finite structure $(T(f g), \phi) \in$ $\mathscr{F}^{r}(T(1: C \rightarrow C))$ on $T(1)=C \otimes C\left(\tilde{S}^{1}\right)$ determined by any finite domination $(D, f, g, h)$ of $C$ coincides with the canonical product round finite structure

$$
(T(f g), \phi)=C \otimes \Sigma^{1} \in \mathscr{F}^{r}\left(C \otimes C\left(\tilde{S}^{1}\right)\right) .
$$

Proof. Let $C^{\prime}=\operatorname{im}(p: E \rightarrow E)$ be the image of a projection $p=p^{2}$ of an even finite chain complex $E$ over $A$ such that there exists a chain equivalence

$$
\theta: C^{\prime} \rightarrow C
$$

The canonical product round finite structure is defined by

$$
C \otimes \Sigma^{1}=(T(p), \psi) \in \mathscr{F}^{r}\left(C \otimes C\left(\widetilde{S}^{1}\right)\right),
$$

with

$$
\begin{aligned}
\psi & =\left(\begin{array}{cc}
\theta q & 0 \\
0 & \theta q
\end{array}\right): T(p)_{r}=E_{r-1}\left[z, z^{-1}\right] \oplus E_{r}\left[z, z^{-1}\right] \rightarrow\left(C \otimes C\left(\tilde{S}^{1}\right)\right)_{r} \\
& =C_{r-1}\left[z, z^{-1}\right] \oplus C_{r}\left[z, z^{-1}\right],
\end{aligned}
$$

where $q: E \rightarrow C^{\prime} ; x \mapsto p(x)$ is the projection.

Let $\Gamma: 0 \simeq 1: \hat{C}(\theta) \rightarrow \hat{C}(\theta)$ be a chain contraction of the modified algebraic mapping cone, so that

$$
\Gamma=\left(\begin{array}{cc}
h^{\prime} & \theta^{\prime} \\
k & -h
\end{array}\right): \hat{C}(\theta)_{r}=C_{r-1}^{\prime} \oplus C_{r} \rightarrow \hat{C}(\theta)_{r+1}=C_{r}^{\prime} \oplus C_{r+1}
$$

with $\theta^{\prime}: C \rightarrow C^{\prime}$ a chain map, $h, h^{\prime}$ chain homotopies

$$
h: \theta^{\prime} \theta \simeq 1: C \rightarrow C, \quad h^{\prime}: \theta \theta^{\prime} \simeq 1: C^{\prime} \rightarrow C^{\prime},
$$

and $k$ such that

$$
h \theta-\theta h^{\prime}=d k-k d^{\prime}: C_{r}^{\prime} \rightarrow C_{r+1} .
$$


Use $\Gamma$ to define a finite domination $(D, f, g, h)$ of $C$ by

$$
\begin{aligned}
& f=q^{\prime} \theta^{\prime}: C \stackrel{\theta^{\prime}}{\longrightarrow} C^{\prime} \stackrel{q^{\prime}=\text { inclusion }}{\longrightarrow} E=D \\
& g=\theta q: D=E \stackrel{q=\text { projection }}{\longrightarrow} C^{\prime} \stackrel{\theta}{\longrightarrow} C \\
& h: g f=\theta^{\prime} \theta \simeq 1: C \rightarrow C .
\end{aligned}
$$

The canonical round finite structure

$$
(T(f g), \phi) \in \mathscr{F}^{r}\left(C \otimes C\left(\widetilde{S}^{1}\right)\right)
$$

is defined by

$$
\begin{aligned}
\phi=S(h) S(g, f)= & \left(\begin{array}{cc}
1 & 0 \\
z h & 1
\end{array}\right)\left(\begin{array}{ll}
g & 0 \\
0 & g
\end{array}\right)=\left(\begin{array}{cc}
g & 0 \\
z h g & g
\end{array}\right): \\
& T(f g)_{r}=E_{r-1}\left[z, z^{-1}\right] \oplus E_{r}\left[z, z^{-1}\right] \\
& \rightarrow\left(C \otimes C\left(\tilde{S}^{1}\right)\right)_{r}=C_{r-1}\left[z, z^{-1}\right] \oplus C_{r}\left[z, z^{-1}\right] .
\end{aligned}
$$

The chain homotopy

$$
e=q^{\prime} h^{\prime} q: f g=q^{\prime} \theta^{\prime} q \simeq q^{\prime} q=p: E \rightarrow E
$$

determines an isomorphism of round finite chain complexes

$$
S(e): T(f g) \rightarrow T(p)
$$

with

$$
\begin{aligned}
S(e) & =\left(\begin{array}{cc}
1 & 0 \\
z e & 1
\end{array}\right): T(f g)_{r}=E_{r-1}\left[z, z^{-1}\right] \oplus E_{r}\left[z, z^{-1}\right] \rightarrow T(p)_{r} \\
& =E_{r-1}\left[z, z^{-1}\right] \oplus E_{r}\left[z, z^{-1}\right],
\end{aligned}
$$

and

$$
\tau(S(e))=0 \in K_{1}\left(A\left[z, z^{-1}\right]\right) .
$$

The diagram of chain equivalences

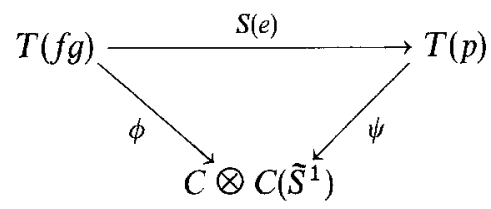

is chain homotopy commutative, with a chain homotopy

$$
j: \psi S(e) \simeq \phi: T(f g) \rightarrow C \otimes C\left(S^{1}\right)
$$


defined by

$$
\begin{aligned}
& j=\left(\begin{array}{cc}
0 & 0 \\
z k & 0
\end{array}\right): T(f g)_{r}=E_{r-1}\left[z, z^{-1}\right] \oplus E_{r}\left[z, z^{-1}\right] \\
& \rightarrow\left(C \otimes C\left(\tilde{S}^{1}\right)\right)_{r}=C_{r-1}\left[z, z^{-1}\right] \oplus C_{r}\left[z, z^{-1}\right]
\end{aligned}
$$

Thus

$$
(T(f g), \phi)-(T(p), \psi)=\tau(S(e))=0 \in K_{1}\left(A\left[z, z^{-1}\right]\right),
$$

and

$$
(T(f g), \phi)=(T(p), \psi) \in \mathscr{F}^{r}\left(C \otimes C\left(\widetilde{S}^{1}\right)\right) .
$$

Given a group $\pi$ and a morphism $\alpha: \pi \rightarrow \pi$, define the group

$$
\pi *_{\alpha} \mathbb{Z}=\pi^{*} \mathbb{Z} /\left\{z^{-1} g z=\alpha(g) \mid g \in \pi\right\},
$$

denoting the generator $1 \in \mathbb{Z}$ by $z$. There is then a natural identification of rings

$$
\mathbb{Z}\left[\pi *_{\alpha} \mathbb{Z}\right]=\mathbb{Z}[\pi] *_{\alpha}\left[z, z^{-1}\right]
$$

and the canonical morphism of rings $i: \mathbb{Z}[\pi] \rightarrow \mathbb{Z}[\pi] *_{x}\left[z, z^{-1}\right]$ is induced by a canonical morphism of groups

$$
i: \pi \rightarrow \pi *_{\alpha} \mathbb{Z} ; \quad g \mapsto g .
$$

There is also defined a morphism of groups

$$
j: \pi *_{\alpha} \mathbb{Z} \rightarrow \mathbb{Z} ; \quad g \mapsto 1, z^{n} \mapsto z^{n}
$$

which is onto, and induces a morphism of rings

$$
j: \mathbb{Z}[\pi] *{ }_{\alpha}\left[z, z^{-1}\right] \rightarrow \mathbb{Z}\left[z, z^{-1}\right]
$$

which is also onto. If $\alpha: \pi \rightarrow \pi$ is an automorphism $\pi *_{\alpha} \mathbb{Z}=\pi \times{ }_{\alpha} \mathbb{Z}$ is the $\alpha$-twisted extension of $\pi$ by $\mathbb{Z}$, with an

$$
\{1\} \rightarrow \pi \stackrel{i}{\longrightarrow} \pi \times \times_{\alpha} \mathbb{Z} \stackrel{j}{\longrightarrow} \rightarrow \mathbb{Z} \rightarrow\{1\}
$$

and

$$
\mathbb{Z}[\pi]_{\alpha}\left[z, z^{-1}\right]=\mathbb{Z}[\pi]_{\alpha}\left[z, z^{-1}\right]
$$

is the $\alpha$-twisted polynomial extension of $\mathbb{Z}[\pi]$. On the other hand, if $\alpha(\pi)=\{1\} \subseteq \pi$ then $i(\pi)=\{1\} \subseteq \pi *_{\alpha} \mathbb{Z}$ and the morphisms $j: \pi *_{\alpha} \mathbb{Z} \rightarrow \mathbb{Z}, j: \mathbb{Z}[\pi] *_{\alpha}\left[z, z^{-1}\right] \rightarrow$ $\mathbb{Z}\left[z, z^{-1}\right]$ are isomorphisms.

PROPOSITION 6.6 (i) Let $f: X \rightarrow X$ be a cellular map to itself of a connected $C W$ complex $X$ with universal cover $\tilde{X}$, and let $\mathrm{f}_{*}=\alpha: \pi_{1}(\mathrm{X}) \rightarrow \pi_{1}(\mathrm{X})$. Then the mapping 
torus $T(f)$ is a connected $C W$ complex with fundamental group

$$
\pi_{1}(\mathrm{~T}(\mathrm{f}))=\pi_{1}(\mathrm{X}) *_{\alpha} \mathbb{Z},
$$

and the cellular chain complex over $\mathbb{Z}\left[\pi_{1}(\mathrm{~T}(\mathrm{f}))\right]$ of the universal cover $T(f)$ is given by

$$
\begin{aligned}
\mathrm{C}(\mathrm{T}(\tilde{\mathrm{f}}))= & \hat{\mathrm{C}}\left(1-\mathrm{z} \tilde{\mathrm{f}}: \mathrm{i}_{!} \mathrm{C}(\tilde{\mathrm{X}}) \rightarrow \mathrm{i}_{!} \mathrm{C}(\tilde{\mathrm{X}})\right) \\
= & \text { the algebraic mapping torus } T(\tilde{f}) \text { of the } \\
& \text { induced chain map } \tilde{\mathrm{f}}: \alpha_{!} \mathrm{C}(\tilde{\mathrm{X}}) \rightarrow \mathrm{C}(\tilde{\mathrm{X}}) \\
& \text { over } \mathbb{Z}\left[\pi_{1}(\mathrm{X})\right] .
\end{aligned}
$$

(ii) A homotopy of maps $\mathrm{e}: \mathrm{f} \simeq \mathrm{f}^{\prime}: \mathrm{X} \rightarrow \mathrm{X}$ induces a homotopy equivalence of mapping tori $S(e): T(f) \rightarrow T\left(f^{\prime}\right)$ and also a chain homotopy $\tilde{e}: \tilde{f} \simeq \tilde{f}^{\prime}: \alpha_{1} C(\tilde{X}) \rightarrow C(\tilde{X})$, such that

$$
\widetilde{S(e)}=S(\tilde{e}): \overparen{C(T}(f))=T(\tilde{f}) \rightarrow C\left(\widetilde{T\left(f^{\prime}\right)}\right)=T\left(\tilde{f}^{\prime}\right) \text {. }
$$

(iii) Let $f: X \rightarrow Y, g: Y \rightarrow X$ be maps, and let

$$
\begin{aligned}
& f_{*}=\alpha: \mathbb{Z}\left[\pi_{1}(X)\right] \rightarrow \mathbb{Z}\left[\pi_{1}(Y)\right], \\
& \mathrm{g}_{*}=\beta: \mathbb{Z}\left[\pi_{1}(Y)\right] \rightarrow \mathbb{Z}\left[\pi_{1}(X)\right], \\
& \tilde{f}: \alpha_{1} C(\tilde{X}) \rightarrow C(\tilde{Y}), \quad \tilde{g}: \beta_{1} C(\tilde{Y}) \rightarrow C(\tilde{X}) .
\end{aligned}
$$

The homotopy equivalence $S(f, g): T(g f) \rightarrow T(f g)$ induces the isomorphism of rings

$$
\begin{aligned}
S(f, g)_{*}=k: & \mathbb{Z}\left[\pi_{1}(T(g f))\right]=\mathbb{Z}\left[\pi_{1}(X)\right] *_{\beta \alpha}\left[z, z^{-1}\right] \\
& \rightarrow \mathbb{Z}\left[\pi_{1}(T(f g))\right]=\mathbb{Z}\left[\pi_{1}(Y)\right] *_{\alpha \beta}\left[z, z^{-1}\right] \\
& a \mapsto \alpha(a), z \mapsto z
\end{aligned}
$$

and the induced chain equivalence is such that

$$
\begin{gathered}
\left.S(\widetilde{f, g})=S(\tilde{f}, \tilde{g}): k_{1} C(\widetilde{T(g f})\right)=k_{1} T\left(\tilde{g}\left(\beta_{!} \tilde{f}\right)\right) \\
\rightarrow C(\widetilde{T(f g)})=T\left(\tilde{f}\left(\alpha_{1} \tilde{g}\right)\right) .
\end{gathered}
$$

Proof. (i) The expression for $\pi_{1}(T(f))$ is the version of the Van Kampen theorem appropriate to the mapping torus construction, and the expression for $C(T(f))$ is the corresponding version of the Mayer-Vietoris presentation.

(ii) \& (iii) follow from (i) and Propositions 6.1 and 6.4 .

Define the canonical round finite structure on the mapping torus $T(\zeta)$ of a self map $\zeta: X \rightarrow X$ of a finitely dominated CW complex $X$ to be the canonical round finite structure on the chain complex $C(\widetilde{T(\zeta)})=T\left(\tilde{\zeta}: \alpha_{1} C(\tilde{X}) \rightarrow C(\tilde{X})\right)$ over $\mathbb{Z}\left[\pi_{1}(T(\zeta))\right]=\mathbb{Z}\left[\pi_{1}(X)\right] *_{\alpha}\left[z, z^{-1}\right]$, with $\alpha=\zeta_{*}: \pi_{1}(X) \rightarrow \pi_{1}(X)$, using the correspondence between the algebraic and the geometric mapping torus of Proposition 6.6. A finite domination $(Y, f, g, h)$ of $X$ determines a (round) finite $\mathrm{CW}$ complex $T(f \zeta g: Y \rightarrow Y)$ and a homotopy equivalence

$$
\phi=S(\zeta h) S(\zeta g, f): T(f \zeta g) \rightarrow T(\zeta)
$$


such that the induced finite domination $\left(\mathbb{Z}\left[\pi_{1}(X)\right] \otimes_{\mathbb{Z}\left[\pi_{1}(\gamma)\right]} C(\bar{Y}), \tilde{f}, \tilde{g}, \tilde{h}\right)$ of $C(\tilde{X})$ determines the (round) finite chain complex $\widetilde{C(T(f \tilde{\xi} g))}=T(\tilde{f} \bar{\xi} \hat{g})$ and the chain equivalence

$$
\tilde{\phi}=S(\tilde{\zeta} \tilde{h}) S(\tilde{\zeta} \tilde{g}, \tilde{f}): T(\tilde{f} \tilde{g} \tilde{g}) \rightarrow T(\tilde{\zeta}),
$$

so that $(T(\tilde{f} \tilde{g} \tilde{g}), \tilde{\phi}) \in \mathscr{F}^{r}(T(\tilde{\zeta}))=\mathscr{F}^{r}(T(\zeta))$ is the canonical round finite structure. We have proved:

PROPOSITION 6.7 The geometric canonical finite structure $(T(f \zeta g), \phi) \in \mathscr{F}(T(\zeta))$ is the reduction of the algebraic canonical round finite structure $(T(\tilde{f} \bar{\zeta} \tilde{g}), \tilde{\phi}) \in \mathscr{F} r(T(\widetilde{\zeta}))$.

In particular, for $\zeta=1: X \rightarrow X$ Propositions 6.5 and 6.7 identify the geometric canonical finite structure on $T(1)=X \times S^{1}$ of Mather [12] and Ferry [6] with the reduction of the canonical product round finite structure $X \times \Sigma^{1} \in \mathscr{F}^{r}\left(X \times S^{1}\right)$. Thus if $(F, \phi) \in \mathscr{F}\left(X \times S^{1}\right)$ is the canonical finite structure the Whitehead torsion of the composition homotopy equivalence of finite $\mathrm{CW}$ complexes

$$
\phi^{-1}(1 \times-1) \phi: F \stackrel{\phi}{\longrightarrow} X \times S^{1} \stackrel{1 \times-1}{\longrightarrow} X \times S^{1} \stackrel{\phi^{-1}}{\longrightarrow} F
$$

is given by Proposition 5.8 (ii) to be the reduction of

$$
\begin{aligned}
& \tau_{X \times \Sigma^{1}}\left(1 \times-1: X \times S^{1} \rightarrow X \times S^{1}\right) \\
& \quad=[X] \otimes \tau(-z) \in K_{1}\left(\mathbb{Z}\left[\pi_{1}(X)\right]\left[z, z^{-1}\right]\right),
\end{aligned}
$$

that is

$$
\tau\left(\phi^{-1}(1 \times-1) \phi\right)=[X] \otimes \tau(-z) \in \mathrm{Wh}\left(\pi_{1}(X) \times \mathbb{Z}\right)
$$

with $[X] \in \bar{K}_{0}\left(\mathbb{Z}\left[\pi_{1}(X)\right]\right)$ the Wall finiteness obstruction. The geometrically defined injection of Ferry [6]

$$
\begin{aligned}
& \bar{B}^{\prime}: \tilde{K}_{0}(\mathbb{Z}[\pi]) \mapsto \mathrm{Wh}(\pi \times \mathbb{Z}) ; \\
& {[X] \mapsto \tau\left(\phi^{-1}(1 \times-1) \phi: F \rightarrow F\right) \quad\left(\pi_{1}(X)=\pi\right)}
\end{aligned}
$$

is thus given algebraically by the variant

$$
\begin{aligned}
& \bar{B}^{\prime}: \tilde{K}_{0}(\mathbb{Z}[\pi]) \nrightarrow \mathrm{Wh}(\pi \times \mathbb{Z}) ; \\
& {[P] \rightarrow[P] \otimes \tau(-z)=\tau\left(-z: P\left[z, z^{-1}\right] \rightarrow P\left[z, z^{-1}\right]\right)}
\end{aligned}
$$

of the original algebraic split injection of Bass, Heller and Swan [2]

$$
\begin{aligned}
& \bar{B}: \tilde{K}_{0}(\mathbb{Z}[\pi]) \nrightarrow \mathrm{Wh}(\pi \times \mathbb{Z}) ; \\
& {[P] \mapsto[P] \otimes \tau(z)=\tau\left(z: P\left[z, z^{-1}\right] \rightarrow P\left[z, z^{-1}\right]\right) .}
\end{aligned}
$$

It is $\bar{B}^{\prime}$ rather than $\bar{B}$ which is geometrically significant. (See Ranicki [22]). 
For example, the trivial $S^{1}$-bundle transfer maps

$$
\phi_{H}^{!}: \hat{H}^{n}\left(\mathbb{Z}_{2} ; \tilde{K}_{0}(\mathbb{Z}[\pi])\right) \longmapsto \hat{H}^{n+1}\left(\mathbb{Z}_{2} ; \mathrm{Wh}(\pi \times \mathbb{Z})\right)
$$

on the Tate $\mathbb{Z}_{2}$-cohomology groups of the duality involutions which appear in the appendix of Munkholm and Ranicki [13] are induced by $\bar{B}^{\prime}$ not $\bar{B}$.

\section{References}

1. Bass, H., 1968, Algebraic K-Theory, Benjamin.

2. Bass, H., Heller, A., and Swan, R. G., 1964, The Whitehead group of a polynomial extension, Publ. Math. I.H.E.S. 22, 61-79.

3. Casson, A. 1973, Whitehead groups of free products with amalgamation, Proc. 1972 Battelle Seattle Conference on Algebraic K-theory, Springer Lecture Notes No. 342, pp. 144-154.

4. Cohen, M., 1973, A Course in Simple Homotopy Theory, Graduate Texts in Mathematics No. 10, Springer.

5. Farrell, F. T., 1971, The obstruction to fibering a manifold over the circle, Proc. I.C.M. Nice 1970, Vol. 2., pp. 69-72.

6. Ferry, S., 1981, A simple-homotopy approach to the finiteness obstruction, Proc. Shape Theory Conference, Springer Lecture Notes No. 870, 73-81.

7. Gersten, S., 1966, A product formula for Wall's obstruction, Amer. J. Math. 88, 337-346.

8. Gersten, S., 1967, The torsion of a self equivalence, Topology 6, 411-414.

9. Hambleton, I., Ranicki, A., and Taylor, L., Round L-theory, J. Pure Appl. Alg., (to appear).

10. Kwun, K. W. and Szczarba, R. H., 1965, Product and sum theorems for Whitehead torson, Ann. Math. 82, 183-190.

11. Lück, W., The transfer maps induced in the algebraic $K_{0^{-}}$and $K_{1}$-groups by a fibration I, Math. Scand. (to appear).

12. Mather, M., 1965, Counting homotopy types of manifolds, Topology 4, 93-94.

13. Munkholm, H. J. and Ranicki, A. A., 1982, The projective class group transfer induced by an $S^{1}$. bundle, Proc. 1981 Ontario Topology Conference, Canadian Math. Soc. Proc. 2, Vol. 2, pp. 461-484.

14. Ranicki, A. A., 1981, Exact Sequences in the Algebraic Theory of Surgery, Mathematical Notes No. 26, Princeton.

15. Ranicki, A. A., 1985, The algebraic theory of finiteness obstruction, Math. Scand. 57, 105-126.

16. Ranicki, A. A., 1985, The algebraic theory of torsion I, Proc. 1983 Rutgers Topology Conference, Springer Lecture Notes No. 1126, 199-237.

17. Ranicki, A. A., 1984, The algebraic theory of torsion III. Lower $K$-theory, preprint.

18. Siebenmann, L., 1965, The obstruction to finding a boundary for an open manifold of dimension greater than five, $\mathrm{PhD}$ thesis, Princeton.

19. Siebenmann, L., 1970, A total Whitehead torsion obstruction to fibering over the circle, Comm. Math. Helv. 45, 1-48.

20. Smith, J., 1981, Complements of codimension two submanifolds II. Homology below the middle dimension, Illinois. J. Math. 25, 470-497.

21. Wall, C. T. C., 1965/6, Finiteness conditions for CW complexes, I. Ann. Math. 81, 56-69, (1965); II. Proc. Roy. Soc. A295, 129-139 (1966).

22. Ranicki, A. A., 1986, The algebraic and geometric splittings of the $K$ - and $L$-groups of polynomial extensions, Proc. Symposium on Transformation Groups, Poznań 1985, Springer Lecture Notes 1217, $321-364$. 